https://helda.helsinki.fi

\title{
Advances in lipidomics
}

\section{Avela, Henri F.}

2020-11

Avela , H F \& Siren , H 2020 , ' Advances in lipidomics ' , Clinica Chimica Acta , vol. 510 , pp.

123-141 . https://doi.org/10.1016/j.cca.2020.06.049

http://hdl.handle.net/10138/332472

https://doi.org/10.1016/j.cca.2020.06.049

cc_by_nc_nd

acceptedVersion

Downloaded from Helda, University of Helsinki institutional repository.

This is an electronic reprint of the original article.

This reprint may differ from the original in pagination and typographic detail.

Please cite the original version. 


\title{
Lipidomics and recent applications in clinical and medical research with ultrahigh performance liquid chromatography-mass spectrometry - A review
}

\author{
Henri F. Avela and Heli Sirén
}

Department of Chemistry, University of Helsinki, P.O. Box 55, FI-00014 University of Helsinki (FI-00560 Helsinki), Finland

${ }^{*}$ Correspondence: Henri F. Avela (MSc), Faculty of Science, Department of Chemistry, University of Helsinki, A.I. Virtasen Aukio 1 (P.O. Box 55), FI-00014 University of Helsinki, Finland, henri.avela@hotmail.com; Heli Sirén (Assoc. Professor, Doc), Faculty of Science, Department of Chemistry, University of Helsinki, A.I. Virtasen Aukio 1 (P.O. Box 55), FI-00014 University of Helsinki, Finland, heli.m.siren@helsinki.fi

\begin{abstract}
Lipids are organic biomolecules, which contribute to energy storing, cellular and subcellular membrane assembly, functionality, signalling, gene expression regulation and surfactant formation. Lipidomics comprises of identification and quantitation of organic lipids, their derivatives and variants.

The present review article combines the literature on glycero-, glycerophospho- and sphingolipids in lipidomics from the years 2017-2019. In addition, a few papers which have promoted advancement in lipidomics are discussed. The primary focus is lipid profiling of biological lipidomic systems with ultrahigh performance liquid chromatography (UHPLC) coupled with mass spectrometry (MS, tandem MS) detection, data handling, and calculations with analytical tools and current statistical methods. Research on in vivo samples includes matrix categories, such as primary blood derivatives (plasma, serum, erythrocytes, and blood platelets), faecal matter, urine, and liver tissues.
\end{abstract}

Keywords: Lipids, liquid chromatography, tandem mass spectrometry; clinical profiling, urine, blood. 


\section{Highlights:}

Compilation of the recent literature on clinical samples; presentation of the accurate methodology. Evaluation of sample preparation techniques in lipid analyses.

Information on separation and identification of neutral, hydrophobic and hydrophilic lipids. Compilation of current statistical methods used in mass spectrometry of glycero-, glycerophosphoand sphingolipids. 
1 Lipidomics and recent applications in clinical and medical research with ultrahigh performance liquid chromatography-mass spectrometry - A review

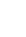

Henri F. Avela and Heli Sirén

Department of Chemistry, University of Helsinki, P.O. Box 55, FI-00014 University of Helsinki (FI-00560 Helsinki), Finland

*) Correspondence: Henri F. Avela (MSc), Faculty of Science, Department of Chemistry, University of Helsinki, A.I. Virtasen Aukio 1 (P.O. Box 55), FI-00014 University of Helsinki,

henri.avela@hotmail.com Finland; Heli Sirén (Assoc. Professor, Doc), Faculty of Science, Department of Chemistry, University of Helsinki, A.I. Virtasen Aukio 1 (P.O. Box 55), FI-00014 University of Helsinki, Finland, heli.m.siren@ helsinki.fi

\section{Abstract}

Lipids are organic biomolecules, which contribute to energy storing, cellular and subcellular membrane assembly, functionality, signalling, gene expression regulation and surfactant formation. Lipidomics comprises of identification and quantitation of organic lipids, their derivatives and variants.

The present review article combines the literature on glycero-, glycerophospho- and sphingolipids in lipidomics from the years 2017-2019. In addition, a few papers which have promoted advancement in lipidomics are discussed. The primary focus is lipid profiling of biological lipidomic systems with ultrahigh performance liquid chromatography (UHPLC) coupled with mass spectrometry (MS, tandem MS) detection, data handling, and calculations with analytical tools and current statistical methods. Research on in vivo samples includes matrix categories, such as primary blood derivatives (plasma, serum, erythrocytes, and blood platelets), faecal matter, urine, and liver tissues. 
25 Keywords: Lipids, liquid chromatography, tandem mass spectrometry; clinical profiling, urine,

26

27

28

29

30

31

32

33

34

35

36

37

38 blood.

Highlights: Compilation of the recent literature on clinical samples; presentation of the accurate methodology; sample preparation; information on separation and identification of neutral, hydrophobic and hydrophilic lipids; compilation of current statistical methods used in mass spectrometry of glycero-, glycerophospho- and sphingolipids.

\section{Contents}

1 Lipidomics

$$
\text { 1.1 Classification of lipids }
$$

$$
\text { 1.2 Lipid types }
$$

1.3 Lipid synthesis in organisms

1.4 Cellular functions of lipids

2 Interpretation of lipidomic profiles

2.1 Profiling of homeostatic systems

2.2 Profiling of metabolic alterations

2.3 Profiling of diseases

3 Sampling and storage for lipid analyses

4 Sample preparation for lipid analyses

5 Liquid chromatography in lipidomics

5.1 High-performance liquid chromatography in lipidomics

5.2 UHPLC systems in lipid analyses

6 Mass spectrometry in lipidomics

6.1 Mass spectrometric analyses of lipids

8 Method optimization

7.1 Lipid classes studied with liquid chromatography Voisi siirtää 5 ja 6 jälkeen? 
8 Lipids important in clinical and medical samples

8.1 Lipids and diseases

9 Metabolite identification with MS and data analysis

10 Untargeted vs targeted approaches

11 Current statistical methods used in mass spectrometry

12 Discussion

13 Conclusions

14 References (1)

\section{Abbreviations}

ACP acyl carrier protein; Cer ceramides; Cer-PC ceramide - phosphatidylcholine; Cer-PE ceramide - ethanolamine; CEs cholesteric esters; DG diglycerols; DB double bond; ESI electrospray ionization; FA fatty acid; GluCer glucosylceramide; GPs glycerophospholipids; GLs glycerolipids; HDL/LDL/VLDL high/low/very low density lipoproteins; HILIC hydrophilic interaction chromatography; LC liquid chromatography; LacCer lactosyl ceramide; LPC lysophosphatidylcholines; LPE lysophosphatidylethanolamine; MS mass spectrometry; MALDI matrix-assisted laser desorption ionization; MG mono glycerols; MGDG monogalactosyldiacylglycerol; MUFA monounsaturated fatty acids; NADPH nicotinamide adenine dinucleotide phosphate; nanoLC nano-liquid chromatography; NEFA/FFA non-esterified fatty acids/free fatty acids; NP normal phase chromatography; PA phosphatidic acid; PG phosphatidylglycerol; PI phosphatidylinositol; PKs Polyketides; PUFA polyunsaturated fatty acids; RP reversed phase chromatography; PRLs prenol lipids; S sphingosine; SA sphinganine; SA1P sphinganine-1-phosphate; SFA saturated fatty acids; SLs saccharolipids; SM sphingomyelin; SPs sphingolipids; SQDG sulfoquinovosyl diacylglycerols; ST cholesterol-based sterols; S1P sphingosine-1-phosphate; PC phosphatidylcholines; PE phosphatidylethanolamine; TG triglycerols; 
TGDG trigalactosyldiacylglycerol; UHPLC-MS ultrahigh performance liquid chromatography-mass spectrometry.

\section{Lipidomics}

Lipidomics is a subgroup within the field of metabolomics with which lipid metabolites can give valuable information about organisms and their biological functions. Lipidomics may be divided into membrane-lipidomics and mediator-lipidomics, [1] which include either comprehensive or quantitative description of membrane lipid constituents or structural characterization and quantification of low abundant bioactive lipid species, respectively. As a sector of metabolomics devoted to the qualitative and quantitative analyses of the lipidome, lipidomics has met an utmost expansion to various biochemical areas. [2] Lipids may be involved in explaining the physiology of multiple interlinked organs, a single organ, biological tissues, individual cells, and biofluids.

In broad terms, lipidomic research covers all aspects of individual lipid species including their structure, function, connection with other cell constituents such as protein, lipid, and metabolite in both health and disease conditions. Details of cell biology obtained from different pathogens (viruses, bacteria, and parasites) provide a great amount of data on molecular structure of host pathogen relations and, consequently, on infection processes [3]. Novelty of lipid analytics is related to reliable, accurate, and sensitive analytical methods for observing new lipid information and correlation with diseases. Abnormalities in lipid metabolism may be an indicator for the stage of a lipidome-related disease, a correlation factor which may be observed when comparing a patient with a healthy individual [4]' [5].

The current review describes identification and determination of lipids in biological, clinical and medical samples with ultrahigh performance liquid chromatography - mass spectrometry (UHPLCMS) in 2017-2019. The publications discuss about determination of biological samples based on (1) chromatography/separation, (2) identification with various types of mass spectrometry, (3) matrices, (4) lipid groups, and (5) mathematical data handling. 


\subsection{Classification of lipids}

According to the definition of lipids made by Fahy et al. [6] lipids are "hydrophobic or amphipathic small molecules that may originate entirely or in part by carbanion-based condensations of thioesters and/or by carbocation-based condensations of isoprene units". [6] Recently, the newest recorded updates of the definition was published by the lipidomics gateway LIPID MAPS® consortium (https://www.lipidmaps.org/data/classification/LM_classification, March 20th 2017). Thus, lipids are characterized according their backbone structure. Lipids include glycerophospholipids (GPs), glycerolipids (GLs), sphingolipids (SPs), polyketides (PKs), cholesterol-based sterols (ST) such as cholesteric esters (CEs), prenol lipids (PRLs) and saccharolipids (SLs). In addition, high-/low-/very low-density lipoproteins (HDL/LDL/VLDL) are frequently studied alongside conventional lipids, though lipoproteins are excluded from this study.

\subsection{Lipid types}

Polar fatty acids (FA) contain fundamental elements of more complex lipids (e.g. for triacylglycerols). They are heteroatom-substituted and esterified conjugates, e.g. acyl carnitines [AcCA] [7]-[10] or bis(monomyristoylglycero)phosphate [BMP] [11], which may include branched or cyclic functionalities [4]. In comparison to the lipid headgroups and hydrocarbon backbones, FA chain function and diversity is less understood. Antonny et al. [12] report several FA saturation gradients: saturated fatty acid glycerophospholipids (SFA GPs) increase the further they are processed in the mammalian cell, namely from the endoplasmatic reticulum (ER) to Golgi apparatus to plasma membrane. [12] Conversely, neural cells have rare sightings of poly-unsaturated fatty acid phosphatidylcholines (PUFA PCs) around the soma, increasing towards the C20:4- and C22:6rich PCs axon end. Higher saturation is often present in uptake-based long FA chains like C22:6-n3, synthesized from C18:3-n3 plant oil (Figure 1). [12]

Lipids get their FA chains either de novo via the Kennedy pathway synthesis [13] or through reacylation (i.e. FA substitution) with available components via the Lands cycle [13]. [12] This 
substitution is enabled through acyltransferase activity whose rate of specificity affects acyl chain diversity.[12] More research on SPs with >C18 FAs is needed. For instance, counter to common belief, SM and Cer include a large abundance of C24:1 and C24:0 FAs. [15]

Glycerophospholipids (GPs) are derived from glycerolipids by substituting a phosphate-linked endgroup to the sn3-carbon of glycerol. GPs have also passive roles as the lipid bilayer cell membrane components. The most abundant lipids in eukaryotic cells are phosphatidylcholines (PC), phosphatidylethanolamine (PE), and their derivatives. [4] Other GP sub-groups include phosphatidic acid (PA), phosphatidylserine (PS), phosphatidylinositol (PI), phosphatidylglycerols (PG), and PG-derived cardiolipins (CL). [4]

GPs in FAs may include ester or ether groups, of which the latter group is defined as plasmalogens (PLs). They cover approximately one fifth of the glycerophospholipids in eukaryotic organisms. [4] PLs are divided into two categories: plasmanyls and plasmenyls. Plasmanyls are noted to have an oxygen bridge (e.g. PI[O-18:0/17:0]) or due to a phosphorus bridge in a case of a plasmenyl group with an ester bond conjugated to a double bond of the FA (e.g. PI[P-18:0, 17:0]). [4] GL and GP structures are depicted in Figure 2 with a list of typical FAs in the mammalian lipidome.

Sphingolipids (SP), characteristic for eukaryotic cells, [15] have a sphingosine base as the backbone (Figure 3). One of these sub-classes are ceramides (Cer), which are lipidic sphingoid bases having nitrogen bonds. One of the SPS is sphingomyelin (SM), which is an interrelated [15] phospho-SP structure that combines ceramide with phosphatidylcholine (Cer-PC) or -ethanolamine (Cer-PE).

\subsection{Lipid synthesis in organisms}

Most lipids are either synthesized in the endoplasmatic reticulum, in Golgi apparatus, and in the mitochondria. [11] Glycerolipids (GL) are mono- (MG), di- (DG) or trisubstituted (TG) glycerols, which belong to common fatty acids having esterifies substituents. [18]

Lipids are primarily formed in FA synthesis with excess of acetyl coenzyme A in presence of NADPH [18] in a cell's cytoplasm. [19] In a model proposed by Nelson and Cox, [18] the synthesis 
150

151

152

153

is a six-step process that involves most notably derivatives formed by acetyl coenzyme A and the acyl carrier protein (ACP). As for the reactants, an initial thioester carbon chain is modified to a malonyl body, which is further lengthened through a catalysed loop of acetyl group addition. The loop includes catalysed reduction steps of thioesters keto-groups (and the leftover double bonds) with NADPH and concludes with releasing of the synthesized FA conjugate base from ACP (either fully saturated or unsaturated). [20] According to Dennis, [18] the formed FAs are typically esterified further near hydroxyl groups in other biomolecules (e.g. glycerol), since the process stabilizes reactive FA. [18] The FA synthesis is biological basis for complicated lipids.

GP metabolism is considered to originate from an enzyme-assisted combination of a FAmetabolism based metabolite (acyl coenzyme A) combined with modified glycolysis products (Dihydroxyacetone phosphate and glycerol), which produces lyso-PA (LPA) further synthesized into PA. [21] As a sidenote, another pathway not going via LPA and PA synthesis is known for synthesizing plasmalogens (PL) [22] and the platelet activating factor (PAF) [23].

For SP synthesis, Cer produced in the ER is translocated and further processed in the Golgi apparatus to produce other SPs. [11] The often identified and quantified sphingosine (S), sphinganine (SA) and their phospholipid metabolites (S1P, SA1P), both of which are important in signalling and central processes alongside LPA and other lipid mediators [24], are considered to be highly potential biomarkers in disease and cancer research. [24]-[26] Pukolski-Gross et al. [26] and Haynes et al. [27] report S1P as the crossroads between reversible dephosphorylation and irreversible catabolism of SP-originated sphingosines into phosphoethanolamine and hexadecenal (in the case of SA1P, hexadecanal), catalysed by sphingosine phosphate lyase. [28] Other sources for more SP metabolism can be found from Kita et al. [24], Sahu et al. [29] and Merrill Jr. et al. [30]. More specifically, articles on Cer metabolism is available by Zheng et al. [31], t'Kindt et al. [32], Castro et al. [33] and Manni et al. [15].

\subsection{Cellular functions of lipids}


Intra- and intercellular lipid metabolism must be understood in its localized context to understand their function in more complex biological systems, e.g. tissue and biofluids. Lipidomic studies often discussed the matters of single cell metabolism and tissues separately, the only link between them being studies on cell signalling [84]. For appropriate tissue analysis, lipid cell function must be understood before drawing conclusions of in-depth lipidomic mechanisms. According to Han ${ }^{[16]}$, more lipidomic pathway flux studies (i.e. reaction rate, metabolic hierarchy and interrelation determinations) are necessary for better understanding lipidomic processes in living organisms ${ }^{[16]}$. For example, the quality of lipids (e.g. lipid class, FA saturation and chain length) in a plasma membrane promote specific lamellar phase properties such as intermolecular order, translational diffusion and elasticity of a cell [11]. A change in these properties is strongly proposed to enable cellular processes like cell budding and tubulation [11] as well as cell fusion and fission. [11], [12], [16]

Van Meer et al.'s [11] extensive reviewed compiled lipidomic observations of cell organelles and membranes in mammals and yeast. Cytoplasmal and extracellular leaflets of double layer membranes have different lipid concentrations and permeabilities: where ER leaflets allow free lipid translocation along its double layer with non-specific lipid transporters, Golgi, plasma membranes and endosomes have restricted lipid mobility [11]. This leaflet asymmetry of specific lipids is proposed to promote special properties for the plasma membrane in question. For translocation of some "trapped" subclass species, lipids are modified enzymatically to enable leaflet flipping (e.g. cytoplasmic leaflet PC is converted to DG in the Golgi apparatus) [11].

Van Meer et al. [11] lists out mechanisms for various means of lipid transportation: lipids can diffuse either within an organelle via the double membrane-specific translocation between leaflets or moved between organelles via tubule, vesicles or nonspecific/specific transport proteins (e.g. the ATP-binding cartridge (ABC) exporters for GP/SP and CERT for Cer-specific transportation) [11]. Basically, eukaryotic membranes are most abundant in PCs (>50\%) and PEs as well as PSs, PIs and 
200 PAs [11]. For major component SPs, SMs are characteristic for mammalian membranes. ${ }^{[16]}$ 201 However, this trend in GPs and SPs is not the case in yeast or other microorganisms since their 202 metabolic pathways differ from eukaryotic [11]. For topical reasons, mammalian metabolism is 203 primarily discussed in this review.

Relative to other organelles, the plasma membrane has a large abundance of SPs, [11] most of 205 which contribute to cell signalling. These lipid classes are either synthesized in the Golgi (SPs) [11]. In addition to its barrier function, the plasma membrane is responsible for cell signalling. [11] Most of the lipids are imported The endoplasmatic reticulum (ER) is the main cell organelle for the 208 bulk synthesis of lipids, especially GPs and cholesterol. [11] Thus, ER is interconnected with many other organelles that synthesize specific lipids [11], [16], [29], [35]: for example the Golgi apparatus. [11] Mostly known as a lipid- and protein-sorting organelle, Van Meer et al. [11] reports the Golgi to be the biggest producer of SPs [11] which are synthesized from ER-produced intermediate Cer. [11] Mitochondria are known for the unique production of cardiolipins (CL), a lipid class proposedly taking part in oxidative phosphorylation. [11], [16] Furthermore, mitochondria are also able to produce LPA for TG, PA and PG synthesis. [11]

Lipid droplets (LDs) are monolayered organelles responsible of TG and cholesterol supply (bound with deployable FFA) [36] for lipid regulation [36] and synthesis as well as energy storage [35],[37],[36], [11]. In addition, LDs are proposed to store lipid signalling precursors and and hydrophobic vitamins. [37] Histologically, LDs consist of a TG and steryl/retinal ester core[36] and phospholipid (PC, PE, PI, LPC, and LPE) membrane. [36] Furthermore, PC availability and lack of its key regulatory enzyme, phosphocholine cytidylyltransferase- $\alpha$ (CTP, Kennedy pathway) [13], are considered integral regulators in LD-growth and -fusion. [35] Since some proteins (articles listed by Fujimoto and Parton) [35] are shared between ER and LDs, [11] not to mention the metabolic regulation between ER-synthesized PA and LD-stored TG usage for lipid synthesis, [37] the two cell organelles are often observed very close to each other. LDs are either positioned in 
proximity, intercalated or membrane stalk-connected with the ER. [35] Similar proximity with LDs has been observed with other lipid-producing organelles [35], [36]: Welte [36] proposes nuclear LDs to affect lipid synthesis via the nucleus, suggesting that LDs regulate transcription factor, enzyme and chromatin availability [36]. Welte [36] also reports LD interaction with mitochondria and peroxisomes [16], [35], both of which are capable of $\beta$-oxidation and FA breakdown [36]. Other studied cell organelle lipidomes include endocytic pathways, namely early and late endosomes, [11] and lysosomes [38].

\section{Interpretation of lipidomic profiles}

\subsection{Structural identification}

Isobaric interference, i.e. co-isolation [39] by co-elution of species with identical or nearly the same molar mass, affects both identification and quantitation in LC-MS [40]. Thus, lipidomic structure profiling and species validation with LC-ESI-MS is mostly done with tandem mass spectrometry $\left(\mathrm{MS}^{\mathrm{n}}\right)$ where more information on class- and species-specific fragmentation can be attained.

For instance, Ivanova et al. [41] reported the characteristic fragmentation of three major lipid classes in positive (Figure 4) and six major lipid classes in negative ESI mode [41]. In positive ESI mode, PC, PS and PE species experience characteristic neutral loss of headgroups as well as fragmentation into lysophosphatidic species. [41] Since negative ESI mode fragmentation seems more complex -maybe more of it is known compared to positive mode- we suggest to read further into GP fragmentation literature by Pi et al. [42] and Ivanova et al. [41] and SP fragmentation by Sullard et al. [43] and Merrill Jr et al. [153] (SP fragmentation depicted in Figure 5).

As a charged particle is moving against an inert gas with known velocity, its movement can be rotationally averaged. Ion mobility spectrometry (IMS) observes this by recording the drift time of an ion species in an electric field. [44] Hence the theoretical CCS value of the analytes can be measured via their drift times with no calibration standards necessarily needed. [44] Some QTOFinstruments are equipped with an IMS module, [45]-[48] enabling further identification tactics with 
250

251

another molecular property dimension. However, identifying CCS values of molecules in ion mobility-mass spectrometry mode (IM-MS mode) decreases the amount of ions ending up at the TOF, which in turn decreases sensitivity in the analyzer. IM-MS may provide valuable information that is otherwise difficult to obtain, since that ion mobility is affected by head group geometry (which is impacted by phosphorylation and glycosylation). [49]

\subsection{Profiling of homeostatic systems}

Determining an organism's lipidome in homeostasis may both act as a fingerprint to the species in question, a distribution of traits in a population as well as a baseline or reference for possible changes of the organism's state. All articles concerning such research were listed in Table 1. As an example, Meulebroek et al. [50] developed a method which may cover all eight lipid classes via faecal matter, using a polarity-switching UHPLC-interfaced Orbitrap. With this, healthy controls and type 2 diabetes patients were monitored and their lipidomic profiles compared with each other. [50] Instead of interpreting as many lipid classes as possible, Manni et al. [15] focused selectively on multiple tissue's and cell culture's 12 Cer:s [51] and sphingomyelins (SMs) in positive mode. Tissue types in this targeted approach included adipose tissue (human, rat), liver (dog, human, rat, mouse), brain (rat, mouse) and serum (human, rat, mouse). [15] For more exotic lipid classes, Drotleff et al. [52] identified and quantified ST hormones from plasma, and, similarly, Gobo et al. [53] cerebral prostaglandins from brain tissue.

Mostly, profiling of human-based biological materials use in vitro cell culture samples and in vivo clinical samples. According to literature, solid and liquid biomatrices, as well as synthesized lipids [54], such as nanoscale liposomes have been studied. The in vitro samples included conditioned cell culture medium (CM, a suspension acquired from cells), and in vivo clinical samples target to e.g. to human amniotic fluid and plasma.

Ghosh and Nisala [5] showed that human tears include at least 600 identified lipids. [5] Thus, for identification and characterization of tears the samples need to be dilute and less diverse than the 
aqueous bioliquids from a multi-cells and complex organisms. In their project, CMs of cell cultures (e.g. from cancer cell lines) were isolated and characterized to evaluate single lipid components in the context of a multivariate tissue. [5] Furthermore, Ghosh and Nishala showed that plasma is a complicated matrix with a large group of GPs, SLs, CEs and TGs. This is understandable, since the bloodstream of an organism is linked to the most of its individual cells, able to transport nutrition, lipids, hormones, cell metabolites, and e.g. dead blood cells. However, Hyötyläinen and Orešič [55] reported that they found a more stable lipid profile in serum than plasma. Hence, a potential alternative for a more reliable analysis could be developed. For plasma, their review suggests differences within sample types, such as lower lipid content in citric acid containing plasma compared to EDTA modified plasma, which might influence the stability of lipids. [55]

\subsection{Profiling of metabolic alterations}

Metabolic profiling of lipids included a discrete comparison of two different diets or other similar analyses between controlled base and altered states of an organism's lipidome (Table 2). In profiling of homeostasis the profiling of metabolic lipids utilizes the main analysis (UHPCMS/MS), investigates the lipid compounds, and studies the dependence and correlation between lipid species and their alterations. Equilibria between lipids in a lipidome may give insight to an organism's metabolic response to its environment. As an example, Zalloua et al. [62] noted a correlation between serum sphingomyelins (SMs) and plasma cholesterol (i.e. LDL, HDL and total cholesterol). [62] metabolite features (m/z 750-810, associated with SMs) expressed a strong correlation to cholesterols.

\subsection{Profiling of diseases}

When triggers concerning a specific lipidomic pathway achieve an extended time to stress the organism, an allostatic load will accumulate (early phase of a clinical condition) until the organism either recovers from the load or fails in the process. Upon failure, a breakdown of the allostatic 
adaptation can be observed as an over- or decompensation of the metabolic pathway biomarkers; a time/concentration threshold essential for clinical diagnostics [78]. Biomarkers studied between 2017-2019 and in the fashion of this disease model were listed in Tables 3-4.

The field of lipidomics has been widely studied for both pathological diagnostics and identification of biomarkers [4]. Mass spectrometry in lipidomics enables discovery, identification, and quantification of lipids in mycobacteria and applications to unravel novel functions of the metabolites. The mycobacteria [81] genus is a group of acidic species characterized by a lipid-rich waxy cell wall, which is much thicker than that found in most other bacteria. Mycobacteria have a high lipid content and complex lipid profile including several unique classes of lipid. Notable members of the genus include Mycobacterium tuberculosis, Mycobacterium leprae, and Mycobacterium ulcerans, causative agents of tuberculosis (TB), leprosy and Buruli ulcers, respectively. In recent study, MS was used to detect mycolic acids in archeological bone samples. Fourteen mycolic acids were detected in a skeleton from the Neolithic period with bone lesions characteristic of skeletal TB. In this case, identification of lipids unique to $M$. tuberculosis offered extra evidence of TB infection to complement diagnoses based on anatomical features and analysis of bacterial DNA. [79] According to Daffe and Draper the amounts lipids in the cell of mycobacteria make up 30-60\% of the dry weight. They have important biological roles, notably in determining virulence and drug resistance. [82] Lipids have importance in mycobacterial infections and they have renaissance in these bacteria.

For ovarian cancer, Perrotti et al.'s [80] review on multiple large studies ( $\mathrm{n}>40)$ strongly suggests an increase of LPLs (specifically LPAs, which have been proposed as early-detection plasma biomarkers) and a change in the FA profile. One explanation for LPL increase was suggested [80]. In addition, Zhang et al. [83] found potential biomarkers in the groups of TGs (decreased), PCs and LPCs (increased). [83] The increased LPC levels caused by "deregulation of phospholipase A2" mentioned by Perrotti et al. [80] Qadir and Malik also reported decreases in TG levels as well as 
325 HDL-cholesterol, cholesterol and LDL-cholesterol of ovarial cancer patients [84]. Furthermore, 326 Perrotti et al. [80] agreed the research of Zhao et al. [86] that GP LPL-profiling can diagnose 327 ovarial cancer in patients [80].

$328 \mathrm{Hu}$ et al. [85] also listed lipidomic studies concerning epidemic disease, cancer, inflammation, 329 dysfunction, genetic disease and drug treatment. Lipidomic data on diseases can also be compared 330 with supporting results of other analyses, as is done for instance with so-called Mendelian 331 Randomization (MR) [87], [88], a statistical-epidemiological method where an risk factor's 332 (biomarker, exposure) causality to an effect (disease) is evaluated. Since genetic influences are not 333 affected by confounding factors (i.e. life choices, environment), a large population of patients can 334 be grouped according to their biomarker-associated genetic variants (i.e. variation in genetic code) 335 and compared with the frequency of a disease in that population. [88] For solid evidence of 336 causality, all the genetic variants must be associated with the biomarker that is studied. [87]

\section{Sampling and storage procedures}

338 Sample preparation needs specific care, since decomposition of chemically stable lipids is 339 considered mainly in the biological way [30]. Directly after isolation from organisms, self340 degradation of lipid compounds initiates via enzyme activation at room temperature. To avoid this, 341 proteins need to be precipitated before analysis which is usually done with methanol or isopropanol 342 [30]. Alternatively, snap freezing of samples is used for storing the authentic samples [114]. Protein 343 precipitation is utilized especially for liquid matrices for improved sensitivity in detection [115].

344 Double bonds of unsaturated fatty acids are susceptible to peroxidation, hydrolytic degradation, and 345 oxidation. Especially, after isolation and extraction, antioxidative properties of biological 346 compounds may be reduced or lost during storing. Major factors contributing to degradation are 347 formation of ice crystals combined with osmotic shock when freezing samples, which decreases the 348 volume of aqueous solution, breaks structures (e.g. cell membranes) between lipids and enzymes 349 which consequently hastens lipid breakdown. [114] 
350

351

352

353

Jurowski et al. [114] reported drastic changes in lipid composition of plasma, when the sample was stored for 1-3 years at $-20{ }^{\circ} \mathrm{C}$. Furthermore, Jurowski et al. [114] observed an increase in concentrations of saturated FAs and decrease of unsaturated polyFAs. The reason for that were enzymes and activity of antioxidants. [114] Furthermore, Hyötyläinen and Orešič [55] informed that a drop in lyso-phosphatidylcholine (LPC) concentrations is detected in less than two hours (storage temperature $4{ }^{\circ} \mathrm{C}$ ). In total, LPC concentrations increased within 24 hours, potentially due to activity of the phospholipid-splicing phospholipase A2. On the other hand, most PCs and sphingolipids seemed to stay stable for this time even at room temperature. [55] Thus, erythrocyte FAs in plasma are already degraded within one week at storage temperatures between $4{ }^{\circ} \mathrm{C}$ and -20 ${ }^{\circ} \mathrm{C}$. Jurowski et al. [114] informed that samples stored at $-60{ }^{\circ} \mathrm{C}$ do not change in one year of storaging. [114] Hyötyläinen and Orešič [55] agreed the stability of lipid containing samples stored at $-80{ }^{\circ} \mathrm{C}$ for at least six months, but a change was seen at a time span of five years. [55] Sampling, sample storage and pre-analysis lipid degradation has been discussed in greater detail by Hyötyläinen and Orešič [55], Patterson et al. [115], Tumanov et al. [116], Jurowski et al. [114] and Monnin et al. [30].

\section{Sample preparation for lipid analyses}

The main limitations of lipidomics analysis are the chemical complexity of the lipids, the range of concentrations at which they exist, and the variety of samples usually analyzed. [117] Especially, sample preparation is needed to prevent lipids at high concentration to suppress ionization of low concentrated lipids in mass spectrometry detection.

Here, only few examples are discussed to show the complexity of the matrices, when HPLC-MS methods are used for information collection of lipids. Altered lipid metabolism plays a key role in the pathogenesis of common diseases. Most of the bioactive mediators originate from the cleavage of lipid constituents of cellular membranes under the activity of phospholipases. In amniotic fluid research, lipids identified were used as indicators for stages of pregnancy or pregnancy 
complications. [5] Total lipid and phospholipid concentrations were observed to increase from 24 weeks of gestation until labour. Thus, routine tests for fatal development evaluation of lecithin/sphingomyelin-ratios could be used for long-term monitoring. Furthermore, lipidome profiles of amniotic fluids were observed to be different between infants born in term and those of pre-term children. [5]

Primary ascites sample matrices have discovered to have abnormal formation of intra-peritoneal fluid in a patient's abdominal cavity. [118] Two types of lipid samples could be used according to formation: non-inflammation induced (e.g. hydrostats- or osmosis-produced) transudates matrix and inflammation-accompanied substances secreted by an organism. Transudates are suspect of decompensation of blood circulation, kidneys, and liver, whereas exudates are formed in oozing of ruptured or otherwise damaged cell tissues formed in complications of cysts in ovarian cancer tumors. In contrast to commonly clear transudates, in intraperitoneal space exudates are noticed to have higher protein-concentrations due to the less filtrated nature. [118]

In the Folch-method [119] about 5\% of proteins are left in the organic phase, when lipids are isolated. That may contribute to matrix effects and higher background noise. [115] In comparison to the Folch method, BUME method [120] is an extraction technique considered superior from the environmental, economic and preparative standpoints, since extraction needs no chloroform and needs less solvent. The method allows to extract all matrices into millilitre volumes with comparatively lowered safety hazards. [121]-[124]

For samples with low lipid concentrations, solid phase extraction (SPE) [58], [125] or lyophilization (i.e. freeze-drying) [125] can be considered to concentrate lipids in a sample for the analysis. Teo et al. [125] introduced a concept of energy-based extraction methods (i.e. pressurized liquid, microwave- and ultrasonic-assisted extraction) alongside polarity-based extraction methods (i.e. single solvent, liquid-liquid, solid phase extraction combined with supercritical fluid extraction which was developed by Jurovski et al. [114] The latter techniques are used more frequently in 
400 lipidomics studies. [125] Energy-based techniques warm the extraction system to achieve a faster 401 and more efficient removal of lipids from the matrices. [125] Table 5 lists extraction and sample 402 purification methods used in lipidomics.

403 Bang et al. [127] conducted solid-phase extraction (SPE) in a superabsorbent polymer (SAP) device 404 for small sample sizes to minimize carry-over of aqueous solvent. In the procedure water was 405 gelated with the SAP polymer, and organic solvent was adsorbed and collected lipids with 406 recoveries similar or greater than in the Folch extraction [127]. Other extraction methods [58], 407 [114], [119]-[125] as well as single solvent and liquid-liquid extractions with acetonitrile or 408 chloroform-methanol 2:1 (v/v) [51], [128] or with methyl-tert-butylether (MTBE, i.e. Matyash) 409 extraction [55], QuEChERS methodology (Quick, Easy, Cheap, Effective, Rugged, and Safe) [127], 410 and the "Bligh and Dyer" method with chloroform-methanol [129]. The use of a chloroform411 methanol solution for lipids extraction was observed to isolate all major lipid classes, which were 412 enriched in the medium polar chloroform phase. [126] The use of MTBE as the solvent led to 413 higher extraction efficiency for unsaturated fatty acids, GPs and Cer, while chloroform-methanol 414 mixture favoured the isolation of saturated fatty acids. [117] However, the recommended sample 415 preparation for isolation of polar lipids in adipose tissue should be combination of liquid-liquid 416 extraction and an SPE to enhance detection of GPs in MS.

\section{Liquid chromatography in lipidomics}

418 Some analytical techniques are specifically better used to identify lipid classes and sample types 419 than individual lipids, why prior knowledge about targeted analysis is to choose the suitable lipid 420 method. According to $\mathrm{Hu}$ et al. [85], NMR techniques can identify and quantify (isolated) lipid 421 fractions as well as provide lipid-associated disease profiles. However, NMR can easily falter in the 422 face of complex sample matrices and their analysis. [85] Apart from desorption and probe MS 423 techniques, imaging techniques lack selectivity for lipid species analysis (though they do have the 424 advantage of nonspecific lipid localization). [132] 


\subsection{High-performance liquid chromatography of lipids}

High-performance liquid chromatography (HPLC) is a method using a liquid mobile phase and solid stationary phase. Analytes are separated depending on the strength of their interaction between stationary phase and mobile phase. Reversed phase liquid chromatography (RP-LC) dominates the chromatography in lipid research and, for that reason, mostly nonpolar and medium-polar lipids are studied. In RP-LC most of the lipid species and internal standards elute at different times, thus experience different matrix effects and different solvent composition, which influences their ionization and may result in inaccurate quantification. [133] However, recent studies with normal phase (NP) stationary packings and with the newest technology in hydrophilic interaction chromatography (HILIC) provides lipid class-specific separation. This has great advantages in terms of quantification, since usually analytes and internal standards show similar retentions. [134] The ultimate advantage is that identification of lipid species with lipid classes is straightforward. Especially, HILIC separation has gained active development recently, since the method separated lipids by distribution, adsorption, ion exchange, and exclusion chromatography. [135] The literature about lipids research with HILIC have slightly increased during the recent years.

Since there is no separation based on the chain lengths of FAs in normal phase chromatography separation, which is the phenomenon in reverse procedures in RP-LC, the precursors affect the retention in the NP column and identification of their MS fragmentation patterns. Full scan MS spectra are usually collected either in negative ionization or in positive ionization mode. Data dependent tandem-MS $\left(\mathrm{MS}^{2}\right)$ was performed in parallel with ESI-Orbitrap-MS scanning on a Fusion mass spectrometer, which is accurate enough for lipid class confirmation. [141] However, identification of chain lengths and saturation states of lipids need high resolution MS instrumentation, when ion resolution is expected further to improve separation efficiency obtained in LC. That was the reason in the study by Jeucken et al. [70], who used a HILIC procedure to observe retention of inorganic ions in lipid matrices. Inorganic ions lead to formation of distorted 
450 lipid adducts in identification with MS. In addition, inorganic salts are shown to decrease 451 repeatability of quantitation, but they are known to co-elute with lipids. [136]

452 Typically, additives are used, since they are dissolved into water or into water-organic solvent

453 mixtures. They allow stabilization of adduct ions concentrations. Additives may form complexes with neutral species due to adduct formation during MS ionization. In lipid analyses, additives have shown to quench and suppress counter-ion species. Furthermore, they show to control the formation of predictable adduct ions, when weaker ligands are present or concentrations of the main ligands are negligible. Moreover, as Erngren et al. [136] have demonstrated with positive mode ionization, inorganic ions improve separation in LC, since the ions are retained onto a normal phase separation column material (such as in HILIC), and they are not co-eluted nor form complex adducts or clusters, thus resulting in decreased repeatability in quantification. [136] This was also the reason for using additives in post-column derivatization by Monnin et al. [30] They compared their LC method performance by using acetic acid and ammonium hydroxide as the additives in the eluent, when detection was done with negative ionization MS. Generally, the study showed that additive ions could form pseudo-neutral adducts with charged lipids by utilization of interaction with the stationary phase via adduct-driven polarity manipulation.

\subsection{UHPLC systems in lipid analyses}

Ultra-high-performance liquid chromatography (UHPLC) was introduced to use fast chromatography with nano-columns [131] Fekete et al. [138] showed that good separation efficiency of lipids at abnormal slow eluent flow through less than $2 \mu \mathrm{m}$ sized particles was possible. The success of the discovery was followed by a near exponential growth of UHPLC and UHPLC-MS publications in the following ten years. Based on Danne-Rasche et al. [140] a Venn diagram is a useful tool to visualize similarities between data from normal and ultra-high LC systems. They discovered that UHPLC and capillary-packed nano-LC (contrary to conventional LC) is necessary for the detection of over half of yeast's lipidome. 
475 Jeucken et al. [70] used UHPLC to separate HeLa-cell lipid metabolites. In their one-step protocol, 476 proteins and other macromolecules were removed and the protein-free chloroform-methanol 477 mixture was injected directly to LC-MS. The extracted lipids were loaded onto a HILIC column 478 [141] During a run time of only 4 min, they achieved separations based on end-groups of lipids 479 (Figure 6). In the study sample preparation of HeLa cells [70] was done on a 96-well plate, on 480 which the caves were filled with the cells and cultivated in the presence and absence of lipid 481 metabolic inhibitors. The studies were done to observe changes in lipidome expression, when the 482 process was altered at the metabolic state.

483

484 485

\section{Mass spectrometry in lipidomics}

The first reports of mass spectrometric (MS) analyses of complex lipid mixtures via soft ionization techniques (matrix-assisted laser desorption ionization (MALDI) and electrospray ionization (ESI)) were developed in the 1990s. [142] In particular, the use of atmospheric pressure ionization (especially electrospray ionization, ESI) and the coupling of liquid chromatography (LC) to MS have allowed the identification and quantitation of ever-increasing numbers of lipid species. Han and Gross [142] showed with ESI-MS that they could make structural determination and even quantitative analyses of individual phospholipid molecular species at sub-picomole level from chloroform extracts of biological samples. In lipidomics mass spectrometry is the only reliable tool for structural identification. Nevertheless, lipidomics is challenging with the many compounds, which cannot yet be expressed as fully as the compounds in genomics and proteomics, which are well represented in various forms at leading research institutions worldwide. [49]

\subsection{Mass spectrometric analyses of lipids}

Lipid analyses from simple profiling to complex metabolite interaction mapping is primarily made with various kinds of ionization techniques in mass spectrometry. Holcapek et al. [143] divided mass spectrometry into three major approaches in lipidomics: direct infusion MS (DIMS, i.e. 
499 shotgun lipidomics, flow injection MS), chromatographic methods on-line coupled with MS, and 500 desorption techniques in MS. [143], [144]

501 DIMS was recommended to be used as a fast-diagnostic method. [186] The direct sample infusion

502 503 504 505 506 507 508 509 510 511 method to MS analyses uses one sample per one run at the time. Thus, it is a very appealing method with multiple precursors and fragments. With DIMS, very high ion resolution and resolving power are needed. Because of many fragment ions continuously present lipid samples are diluted before injection to MS to avoid detector saturation. Furthermore, knowledge on spectral interference is good to be available for quantitation data and considering identification of similar compounds, like identical isomer fragments. [144] Furthermore, matrix effects may play a large role in analyses. [146] Anyhow, desorption techniques are ideal mass-spectrometric imaging of tissues and cells. [143] When locations of the origin of lipids are not possible in conventional LC-MS, desorption DIMS offers a fast solution. For this localization of lipids, multiple approaches have been attempted such as mass spectrometric imaging (MSI) [111], [143], [144], [147] liquid extraction surface analysis (LESA) [140], [148] and probe research [147]-[149]

Solvents are very important in MS analyses of lipids (Monnin et al. [30]). Ammonium hydroxide acetic acid composition were studied in aqueous solution to optimize degree of ionization of lipids in negative ESI-MS. The tests were made for lipids in the classes PA, PE, PC, LPC, LSM, Cer, LPA, and PS. [30] Usually, in positive ESI-MS water-methanol and water-acetonitrile solutions are used with modification of formic acid or acetic acid. [150] Lorenzen et al. used water, acetonitrile, and isopropanol as the eluents in UHPLC-MS/MS studies and identification with both negative and positive electrospray ionization modes. The eluents ( $\mathrm{pH}$ 5.5) contained also ammonium acetate and formic acid as a buffering additives in order to maintain the lipids in the same ionic state during the separation process. [151] To gain all metabolites of a subclass, Griffiths and Wang [188] singleextract STs with ethanol to acquire oxysterols esterified with sulphuric acid and linked sugars. [188] 
523 Table 6 lists examples of lipid classes and molecular species formed during electrospray ionization 524 in negative ESI-MS mode. The fragments used for lipid class identification were $[\mathrm{M}+\mathrm{H}]^{+},[\mathrm{M}+$ $525 \mathrm{Na}^{+},\left[\mathrm{M}+\mathrm{NH}_{4}\right]^{+},\left[\mathrm{M}-\mathrm{H}_{2} \mathrm{O}+\mathrm{H}\right]^{+}$in the positive mode and $[\mathrm{M}-\mathrm{H}]^{-},[\mathrm{M}+\mathrm{HCOO}]^{-}$, and $[\mathrm{M}+$ $\left.526 \mathrm{CH}_{3} \mathrm{COO}\right]^{-}$in the negative mode. [152] Cajka et al. [152] noticed in their project that PEs were the 527 only detectable glycerophospholipid species, which were distinguished by the $[\mathrm{M}-141+\mathrm{H}]^{+} \mathrm{MS}^{2}$ 528 fragment resulting from the neutral loss of the polar end-group. Isolation of lipids from cells was 529 made according to Bligh and Dyer [129] and with reverse-phase liquid chromatography for sample 530 introduction to MS.

531

\section{Method optimization protocols}

For understanding a metabolic-lipidomic system, the lipidome or the relevant lipid group should be mapped out. Merrill Jr. et al. [153] designed a workflow for sphingolipid (SP) analyses of a lyophilized cell culture by using multiple LC-MS/MS protocols for specific subclasses. [153] However, performing comprehensive analyses of lipid polarity ranges is still not sought for until today.

To help method optimization, there are four protocols that inform distinct target groups in analyses. They deal with (1) the extraction procedure for the preparation of sphingolipids for analysis by LC MS/MS, (2) reverse-phase LC, (3) normal-phase LC, and (4) the order of steps to follow in setting up an LC-MS/MS for a new biological sample. Furthermore, they mentioned the relation between sphongolipids (SPs) and their metabolites, which remind the biochemical equilibrium present in a biological system.

\subsection{Lipid classes studied with liquid chromatography}

Teo et al. [125] studied various biofluids and tissues that were analyzed with different chromatographic and ionization methods with mass spectrometry. LC-MS is necessary, when comprehensive screening of biomarkers is required (e.g. in cancer research[137]). Primarily, GLs, GPs, SPs, and STs in liquid-type biological materials (e.g. blood and secretions) and solid tissues 
548 (e.g. eyeball, fibroblasts and skin) were studied with LC-MS. [125] Later, Jurovski et al. [114] 549 showed that FAs and CEs are also important lipids to study clinical samples, when there is a need to 550 identify the lipid profiles.

551 Since variation of lipid classes is enormous, it is not surprising that the polarity of lipid species 552 varies a lot. Figure 7 demonstrates the lipid-subclass ranges of four distinct chromatographic 553 approaches, namely lipids separated in reverse phase and normal phase LC separation strategy. The 554 subclasses include to analyse separately both nonpolar and polar lipids with liquid chromatography 555 separation. Based on the structural differences, the lyso-forms are more polar than their 556 counterparts, which have an extra fatty acid chain in the structure. Particularly, polar and mid-polar (PS, PG, PI, Cer) lipids seem to be species that are often identified with negative mode in MS,

though more species are primarily found with positive mode identification. As to the GLs groups, only MG has a fatty acid chain that is small enough to be rather polar. As a thumb rule, SPs are mid-polar lipids, since they are amines and have nitrogen-bound fatty acid chains.

\section{Lipids important in clinical and medical samples}

Lipids have important roles in the profiling of cell types (e.g. cancer cell lines or bacteria [114]) as well as directly from various biological fluids and biotargets (e.g. plasma [45], [112] and human amniotic membrane [154]). When cells are the examined matrices, they are usually harvested by ultracentrifugation, washed with water, resuspended, incubated, extraction phase isolated with centrifugation, re-extracted, filtered, dried under nitrogen, and dissolved in methanoldichloromethane-water mixture $(35: 70: 5, \mathrm{v} / \mathrm{v} / \mathrm{v})$ [151], though sample preparation methods vary. Tables 7, 8, and 9 list samples analyzed between 2017 - 2019 in lipidomic UHPLC/MS research. Cell lipids are important biomolecules for diseases due to metabolism.

The range of unconventional biological extracts and tissues, as described by Jurovski et al. [114], is very broad. Furthermore, in vitro biological cell culture analyses reached a high variation of studies 
among themselves, which lead to include them separately in Table 9. The multi-matrix approach was a pool of plasma, urine and faecal matter of the same patient. [155]

Tables 10 and 11 list all lipids in the articles where identification of various lipid groups was discussed. Just like fatty acyls (FAcyls, including FAs, BMPs and CLs) were reported for physiological profiling [50], [53], [58], [107], lipido-metabolomic [67], [69], [70], and disease biomarker studies [5], [9], [95], [98]-[100], [102], CEs were frequently found in method development [46], [108], [130], [155], [189] and cancer research. [46], [108], [110], [113] Also, sub-groups, the totality of identified lipids, and biomarker lipids were included. In total, a range of 5-700 total lipids and 5-87 proposed biomarkers were discussed in the articles. Typical GLs in articles included DGs and TGs, with MGs or BMP. [69]

Profiling lipid metabolites of hepatocellular carcinoma revealed down-regulation of TGs having less than two double-bonds in their structures, which was also observed in ceramides. Conversely, up-regulation was noticed for only GPs and SM. Particularly, new information on TG, PC, PE, and PI trends was uncovered. [111] Lipid profiles differ a lot from sample to sample, though some consistencies remain. During negative mode ESI-ionization, Tumanov et al. [116] claimed to have observed a bias in LPA quantitation. Noteworthy is that the concentration of LPAs may be easily overestimated due to typical over-abundance of LPCs combined with the choline moiety which is lost during ionization process in MS. [116]

\section{Metabolite identification with MS and data analysis}

The experiments with both positive and negative ionization modes in MS are useful in lipid analyses, since the data can be combined in order to identify the maximum possible number of lipids. [151] Usually, lipid standards are needed in order to evaluate the chromatographic profiles and fragmentation behavior of lipid species. Comprehensive characterization and quantification of molecular lipid species was shown to be achieved by spiking the lipid extracts with unique lipid 
596

597

598

599

600

601

602

603

604

605

606 607 been published. [169]-[172]

608 When lipids were studied with UPLC-ESI-MS with eluents containing ammonium acetate and 609 formic acid, PGs, PEs, and CLs were detected as [M-H]- ions, and Cers being both hydroxylated 610 and no hydroxylated as $[\mathrm{M}+\mathrm{HCOO}]-$ ions in negative ion mode. In positive ionization mode PEs, 611 Cers, MGs, DGs, and TGs could also be detected, but the former two were detected as $[\mathrm{M}+\mathrm{H}]+$ ions 612 and the latter two as [M+NH4]+ ions. [151], [173]

613 Research on routine in vivo analyses of samples included six categories of matrices, primarily blood 614 (plasma, serum, erythrocytes and blood platelets), faecal matter, and urine. One of the revised 615 papers even proposed a multimatrix analysis by pooling plasma, faecal matter, and urine in one 616 sample to increase method repeatability and lipid coverage. [156] Unconventional biological 617 materials, i.e. harder-to-get as in vivo samples of 18 different biofluid and tissue types, of which the 618 most frequent topic of interest was liver tissue. [15], [63], [111] [127], [155], [160] In addition, 619

standards and using selective ionization conditions for sample infusion to have structure-specific mass analyses by mass spectrometry.

Liebisch et al. [39] proposes two ways for solving the lack in identification: Either mass accuracy must be increased to separate two nearly identical species or fragmentation of $\mathrm{MS}^{\mathrm{n}}$ must be developed. Thus, fragmentation behaviour of PI-Cers (GIPC-Cer) have been described previously and structures were characterized to have a polar head group, fatty acid, and sphingoid base [151], [162], [163]. Ceramide molecules form distinct domains in the cell membrane, which may serve to re-organize cellular receptors and signalling molecules [164]. ESI-MS data for identification and characterization of the structure of PGs, PEs, CLs, and glycerolipids (DGs and TGs) are also available. [164]-[168] The presence of PGs, PEs, and $\mathrm{C}_{26}$-dihydro Ceramide (Cers), as well as the presence of distinctive $\alpha$-hydroxylated FAs together from complete FA profiles of strains have also 
620

621

622

623

624

Moreover, a new way for improved identification and quantitation in lipidomic studies was found:

The utilization of the lipidome isotope labelling of yeast (LILY) standard to produce (nonradioactive) isotopically labelled eukaryotic lipid standards in yeast for normalization and quantification in mass spectrometry. [174] When looking at analytes of low concentrations, higher mass accuracy is often preferred to exchange lower resolution and to increase sensitivity.

A slight trend of multiple studies on big lipid structures (e.g. cell cultures, exosomes, [98], [110] lipid droplets, [60] and liposomes [159]) can be observed. Partially, this can be explained with new trending asymmetric flow field flow fractionation (AF4) [96], [97], [110] instruments, which has ability to separate precisely biomolecules by mass into smaller fractions before primary analysis. In UHPLC-MS lipidomics, the use of a pooled quality control (QC) sample in the analysis sequence has been standardized, which mean that according to the literature search in the year 201711 papers from 25, in 201814 papers from 22, and in 20198 papers from 10 showed the trend of increased pooled sample usage (even in qualitative analyses). [150]

\section{Untargeted vs targeted approaches}

It is currently not possible to comprehensively measure the lipidome of a cell or tissue in a single experiment. Either the precise alteration in lipids to expect is not known in any given case. Thus, the first studies are often exploratory, which means that they are done using untargeted profile monitoring. Thus, the MS methods need high mass accuracy and resolution. Analyses of fragmentation pathways has led to a detailed understanding of the connections between different building blocks found in lipids (such as fatty acids, sphingoid bases, and head groups. Precursor lipids are determined based on characteristic fragment ions, as seen in Figure 8. Other targeted approaches based on tandem mass spectrometry are now available for analysis of many different classes of lipids and in complex mixtures. [175], [176]

Lipids have some unique advantages and challenges for mass spectrometric analyses. The application of electrospray ionization to crude lipid extracts without prior fractionation is one such 
645 646 647 648 649 650 651 652 653 654 655 656 657 658 659 660 661 662 663 664 665 666 667 the biochemical/physiological context of metabolic pathways [179]. In contrast, untargeted 668 approaches need more careful experimental MS data and more data modifications, but also pre669

example, as it has perhaps been more successfully applied in lipidomics than in any other discipline.

The diverse molecular structure of lipids means that collision-induced dissociation alone may be limited in providing unique descriptions of complex lipid structures. [176]' [177]

A conventional targeted approach needs a lot of calibration standards in lipidomics. [50] It is not feasible to calibrate all lipids individually. Thus, a simpler compromise on class-representative lipid standards with optimally chemical similarities are often applied in lipidomic studies. Ideally, however, standards and analytes should be chemically equivalent.

Though expensive, the most used internal standards are deuterated standards, most often by Avanti Polar Lipids (Alabaster, AL, USA). Typically, the protons are deuterated at the FA end of the carbon chain with either 7 or 9 deuterium atoms. For representative standards in analysis, a selfmade or commercial standard mixture is typically used (e.g. a SPLASH mix [130] or well-known organic standard [porcine brain, chicken egg, e. coli] [161]). The molecular ion and its fragments that still include the deuterated FA chain experience a noticeable shift in their $\mathrm{m} / \mathrm{z}$ value compared to their non-deuterated counterparts. Since fragmentation is identical for both variants, this $\mathrm{m} / \mathrm{z}$ differentiation is ideal for ISTD normalization via EICs. [174]

\section{Current statistical methods used in mass spectrometry}

Targeted and untargeted methods are two distinctively different approaches, as well as the means of data processing. Targeted data processing of metabolites was divided to five phases: (1) the acquisition of raw data, (2) the contemplation of which database should be used when considering the research question and analytes, (3) the pre-processing and identification of these metabolites, (4) the normalization and quantification of the identified species, and (5) the reflection of the results on processing for detection. In addition, resolution in screening and identification of the relevant 
670 biomarkers from the totality of features/peaks need to be considered, although the non-targeted 671 steps include the same pathway elucidation as in the targeted analyses [179].

672 In lipidomic research, statistical methods have enlarged into a broad variety of numerical tests and 673 visualization techniques. Typically, statistical methods include a null hypothesis to test similarities 674 by using one dataset. However, the p-value parameter in the Student's t-test, [56], [57], [64], [65], 675 [67]-[69], [72], [73], [75], [76], [92], [97], [98], [94], [102], [104], [105], [127], [156], [159] the 676 non-parametric Mann-Whitney U-test, [56], [74], [76], [93], [99], [103], [104]-[106], and the 677 analysis of variance test (ANOVA) [63]-[65], [98], [107], [109], [113], [156], [157] are for 678 comparison of two or more datasets. These methods are often used for validation of analytical data 679 and for detection of changes between controls and authentic samples. Correction methods such as 680 the Benjamini-Hochberg test [180] are used to calculate the false discovery rate (FDR), i.e. 681 minimizing false positive data from the dataset. [74], [75], [76], [105], [106], [181] An unequal 682 variance test like the Storey [74], [182] or Welch [87] t-test may also be used. [74], [87]

683 The Mann-Whitney U-test [183] is used in mathematical data handling, when all parameters are 684 variables to evaluate similarities of two independent dataset medians with a null hypothesis. For 685 two sets with same or different sample sizes, the values of both datasets are sorted from the smallest 686 to the largest ones. A Mann-Whitney U-test can be fitted for non-Gaussian distribution data. It is 687 typically combined with a Benjamini-Hochberg (or Bonferroni-Holm [69]) test to exclude false 688 positive values, thus giving the false discovery rate (FDR). These methods were applied in multiple 689 studies to limit uncertainty in the results of lipids. Furthermore, Paepe et al. [156] and Gong et 690 al.[105] used cross-validated ANOVA test (CV-ANOVA) to improve reliability of the identified 691 analytes.

692 Lately, Tietz-Bogert et al. [93] calculated the FDR value in a lipido-metabolomic study by 693 searching significant biomarkers of primary sclerosing cholangitis, [93] which is a disorder of lipids 694 in the bile duct of unknown origin. The concentrations of lipid species in control samples of healthy 
695 individual's and the clinical samples of sick patient's blood and bile were compared to find changes 696 with a statistical significance of 0.01. [93]

697 Machine learning and software advancements for multi-ionic identification have allowed to 698 evaluate MS spectra produced with UHPLC-MS in silico, which is only demonstrated in a few 699 recent articles. [47], [57], [90], [113], [161] Due to machine learning techniques and their 700 discovered use in omics, automated lipidomic analyses, ROC/AUC cross-validation analyses, [90], 701 [105], [181] random forest studies, neural network applications, [184] in silico spectra evaluation 702 [161], and CCS value [47] generation algorithms have become in use. More detailed information is 703 available in ref. [132]. search. LipidBlast was mostly developed with ion trap tandem mass spectra, but it can be used with data from other platforms such as QTOF mass spectrometers.

The LipidBlast library works with low-resolution and high-resolution instruments. [185] An $\mathrm{m} / \mathrm{z}$ precursor-ion filter first filtered the data, and a subsequent product-ion match generated a library hit score that reflects the level of confidence for compound annotation. [185] It must be noted that a "closest-match" bias can be apparent when identifying an analyte with a library search. [187], [188] As the library may be incomplete, possible isomers must be considered when validating data.

\section{Discussion}

Lipid function on the tissue-level is rarely explained via cell-level mechanisms, which are the only frequent mechanisms mentioned being lipid signaling. In speculations, lipids may be distributed between tissue cells via vesicles or other means of expulsion depending on the tissue. Do the wellestablished tissue types (epithelium, muscle, connective/protective and nervous tissue) have interor intra-class uniformity or variations in lipid function, which could be supported by cell-level observations? 
720 Due to the structural complexity of lipids, their quantification is not easy to perform. Different 721 strategies and techniques should be addressed depending on the targeted analytes. The difference of ionization efficiency between lipid species, depending on their structure, is indeed a real limitation of this technique.

An increasing number of studies report about poor quality of lipidomics data with misidentification and inaccurate or inappropriate quantification of lipid molecules. These studies primarily use standardless [145] and untargeted metabolomics approaches. [170] To all intents and purposes, the reasons for the poor data quality seem to be analytical, bioinformatics, and educational aspects. Therefore, it is necessary to implement reporting standards for lipidomics data to share with the scientific community [39], [134]. These standards need to cover both shotgun and HPLC-MS approaches. Only applying both approaches in a complementary and confirmatory way permits a comprehensive and accurate coverage of the lipidome, as earlier was documented by G. Liebisch [134].

Plasma membrane asymmetry of two phospholipid leaflets could have implications for membrane potential as well as phase properties (e.g. signalling, diffusion and biochemical activity). Possible mechanisms, such as change in lipid composition or shift in leaflet asymmetry by local synthesis or lipid transportation could be an interesting subject for lipidomic research. Evidently, in real time this would need improvements in the selectivity of in situ lipid imaging techniques, which may possibly be desorption MS [111], [143], [144], [147], or Raman [190], [60], [150] technology in the future.

Some interpretation of the data in the low-abundance field may lead to over speculation. Han [16] proposes small amounts of lipids to be originated from leftover reactants of imperfect biochemical reactions, since virtually no perfect thermodynamic conversion from precursors to products exists. For example, when LPE and LPC is observed in a matrix, PA-derived GP species must be present from de novo plasmanyl synthesis. [16] 
Antonny et al. [12] reminds that FA chain function of lipid classes is less understood, which would insinuate more separation by reversed-phase chromatography. Traditionally, nonpolar and mediumpolar lipids are studied with non-polar phases and two-component mobile phases made of water or buffered water and organic solvents in RP-HPLC. The advantage of ternary mobile phase compositions made of medium polar organic solvents, water or buffered water, and methanol or acetonitrile mixtures would be needed to separate and elute also polar lipids. In addition, the possibility of sequentially performed NP-HPLC and RP-HPLC coupled with MS should be more studied for lipids in biological samples. On the other hand, Liebisch et al. [39] promotes NP-HPLC and HILIC quantification over RP-HPLC for providing polar group selectivity by class selective separation and, thus, internal standard co-elution. [39]

\section{Conclusions}

The paper shows the fact that new methods for lipids and applications in clinical and medical research are still challenging in life science. To obtaining a truly complete overview of all lipids in a sample has remained very challenging due to their enormous structural diversity. A combination of sample extraction and separation procedures is required. Given the central role of lipids as key metabolites with remarkably diverse biological roles, the field of lipidomics may follow a trajectory comparable to the developments seen in genomics and proteomics over the past decade.

This review compiles recent lipidomic studies from the angle of UHPLC-MS research from the past three years (2017-2019). Identified lipids by subclass, biomarkers, clinical and in vitro sample matrices (i.e. biological materials) as well as specified topics on homeostatic, metabolic and disease-metabolic profiling are represented alongside quintessential analytical, biological and chemical insight for data interpretation. As an example, cell tissue data in research has rarely been put into context with cell-level observations. So far, signaling is seen as an only connection between these levels of biological organization.

\section{Acknowledgements}


770 We want to thank the Institute of Analytical Chemistry at the Johannes Kepler University Linz for 771 the support in effort to understand lipidomic research. Mr Joel Tiitinen is also acknowledged for 772 drawing the graphical abstract.

774 Funding: This research received no external funding.

775 Conflicts of Interest: All authors declare no conflict of interest.

\section{References}

778

[1] Serhan, C. N., \& Savill, J. (2005). Resolution of inflammation: the beginning programs the end.

$779 \quad$ Nature immunology, 6(12), 1191.

780 [2] Ståhlman, M., Ejsing, C. S., Tarasov, K., Perman, J., Borén, J., \& Ekroos, K. (2009). High-

781 throughput shotgun lipidomics by quadrupole time-of-flight mass spectrometry. Journal of

782 Chromatography B, 877(26), 2664-2672.

783 [3] Koriem, K. M. M. (2017). A lipidomic concept in infectious diseases. Asian Pacific Journal of 784 Tropical Biomedicine, 7(3), 265-274.

785 [4] LIPID MAPS: Tutorials and Lectures on Lipids, Categories of Lipids. 02.10.2018 786 http://lipidmaps.org/resources/tutorials/lipid_tutorial.php\#FA

787 [5] Ghosh, A., \& Nishtala, K. (2017). Biofluid lipidome: a source for potential diagnostic 788 biomarkers. Clinical and translational medicine, 6(1), 22.

789 [6] Fahy, E., Subramaniam, S., Murphy, R. C., Nishijima, M., Raetz, C. R., Shimizu, T., Spener, F., 790 van Meer, G. , Wakelam, M. J. O., \& Dennis, E. A. (2009). Update of the LIPID MAPS 791 comprehensive classification system for lipids. Journal of lipid research, 50(Supplement), 9-14.

792 [7] Ribeiro, M. A., Murgu, M., de Moraes Silva, V., Sawaya, A. C., Ribeiro, L. F., Justi, A., \& 793 Meurer, E. C. (2017). The screening of organic matter in mineral and tap water by UHPLC-HRMS. 794 Talanta, 174, 581-586. 
795 [8] Rombouts, C., De Spiegeleer, M., Van Meulebroek, L., De Vos, W. H., \& Vanhaecke, L. 796 (2019). Validated comprehensive metabolomics and lipidomics analysis of colon tissue and cell 797 lines. Analytica Chimica Acta.

798 [9] Schlotterbeck, J., Chatterjee, M., Gawaz, M., \& Lämmerhofer, M. (2019). Comprehensive

MS/MS profiling by UHPLC-ESI-QTOF-MS/MS using SWATH data-independent acquisition for the study of platelet lipidomes in coronary artery disease. Analytica chimica acta, 1046, 1-15.

[10] Taylor, N., White, T., \& Viant, M. (2017). Defining the baseline and oxidant perturbed lipidomic profiles of daphnia magna. Metabolites, 7(1), 11.

[11] Van Meer, G., Voelker, D. R., \& Feigenson, G. W. (2008). Membrane lipids: where they are and how they behave. Nature reviews Molecular cell biology, 9(2), 112.

[12] Antonny, B., Vanni, S., Shindou, H., \& Ferreira, T. (2015). From zero to six double bonds: phospholipid unsaturation and organelle function. Trends in cell biology, 25(7), 427-436.

[13] Gibellini, F., \& Smith, T. K. (2010). The Kennedy pathway-de novo synthesis of phosphatidylethanolamine and phosphatidylcholine. IUBMB life, 62(6), 414-428.

[14] Wang, L., Shen, W., Kazachkov, M., Chen, G., Chen, Q., Carlsson, A. S., ... \& Zou, J. (2012). Metabolic interactions between the Lands cycle and the Kennedy pathway of glycerolipid synthesis in Arabidopsis developing seeds. The Plant Cell, 24(11), 4652-4669.

[15] Manni, M. M., Sot, J., Arretxe, E., Gil-Redondo, R., Falcón-Pérez, J. M., Balgoma, D., Alonso, C., Goñi, F. M., \& Alonso, A. (2018). The fatty acids of sphingomyelins and ceramides in mammalian tissues and cultured cells: Biophysical and physiological implications. Chemistry and physics of lipids, 217, 29-34.

[16] Han, X. (2016). Lipidomics for studying metabolism. Nature Reviews Endocrinology, 12(11), 668.

[17] LIPID MAPS, Databases: "Classification-based search", Lipid Categories, 22.11.2018, http://lipidmaps.org/resources/databases/index.php 
820

821

822

823

824 825

826

827

828

829

830

831

832

833

834

835

836

837

838

839

840

841

842

843

844

[18] Dennis, EA: “LIPID MAPS Lipid Metabolics Tutorial, Fatty Acid Biosynthesis”, Powerpoint, 2010, University of California, San Diego

[19] Wakil, S. J., Stoops, J. K., \& Joshi, V. C. (1983). Fatty acid synthesis and its regulation. Annual review of biochemistry, 52(1), 537-579.

[20] Nelson DL, Cox MM (2005), Lehninger Principles of Biochemistry, $4^{\text {th }}$ ed., W.H. Freeman \& Co.

[21] Manzo-Fontes, A., Slenter, D., Willighagen, E., Kutmon, M., Cirilla, E., “Glycerophospholipid Biosynthetic Pathway (Homo sapiens)” WikiPathways, 1.11.2019, link checked 6.11.2019, https://www.wikipathways.org/index.php/Pathway:WP2533

[22] Nagan, N., \& Zoeller, R. A. (2001). Plasmalogens: biosynthesis and functions. Progress in lipid research, 40(3), 199-229.

[23] Detopoulou, P., Nomikos, T., Fragopoulou, E., Antonopoulou, S., Kotroyiannis, I., Vassiliadou, C., ... \& Stefanadis, C. (2009). Platelet activating factor (PAF) and activity of its biosynthetic and catabolic enzymes in blood and leukocytes of male patients with newly diagnosed heart failure. Clinical biochemistry, 42(1-2), 44-49.

[24] Kita, Y., Tokuoka, S. M., \& Shimizu, T. (2017). Mediator lipidomics by liquid [25] Scherer, M., Schmitz, G., \& Liebisch, G. (2009). High-throughput analysis of sphingosine 1phosphate, sphinganine 1-phosphate, and lysophosphatidic acid in plasma samples by liquid chromatography-tandem mass spectrometry. Clinical chemistry, 55(6), 1218-1222.

[26] Pulkoski-Gross, Michael J., Donaldson, J. C., Obeid, L. M., "Sphingosine-1-phosphate metabolism: A structural perspective." Critical reviews in biochemistry and molecular biology 50.4 (2015): 298-313.

[27] Haynes, C. A., Allegood, J. C., Park, H., \& Sullards, M. C. (2009). Sphingolipidomics: methods for the comprehensive analysis of sphingolipids. Journal of Chromatography B, 877(26), 2696-2708. 
845 [28] Lydic, T. A., \& Goo, Y. H. (2018). Lipidomics unveils the complexity of the lipidome in 846 metabolic diseases. Clinical and translational medicine, 7(1), 4.

847 [29] Sahu, S. K., Hannun, Y. A., \& Yao, N. (2019). Emergence of membrane sphingolipids as a 848 potential therapeutic target. Biochimie.

849 chromatography-tandem mass spectrometry. Biochimica et Biophysica Acta (BBA)-Molecular and 850 Cell Biology of Lipids, 1862(8), 777-781.

851 [30] Monnin, C., Ramrup, P., Daigle- Young, C., \& Vuckovic, D. (2018). Improving negative 852 liquid chromatography/electrospray ionization mass spectrometry lipidomic analysis of human 853 plasma using acetic acid as a mobile- phase additive. Rapid Communications in Mass 854 Spectrometry, 32(3), 201-211.

855 [31] Zheng, W., Kollmeyer, J., Symolon, H., Momin, A., Munter, E., Wang, E., ... \& Ramaraju, H. 856 (2006). Ceramides and other bioactive sphingolipid backbones in health and disease: lipidomic 857 analysis, metabolism and roles in membrane structure, dynamics, signaling and autophagy. 858 Biochimica et Biophysica Acta (BBA)-Biomembranes, 1758(12), 1864-1884.

859 [32] t'Kindt, R., Jorge, L., Dumont, E., Couturon, P., David, F., Sandra, P., \& Sandra, K. (2011). 860 Profiling and characterizing skin ceramides using reversed-phase liquid chromatography861 quadrupole time-of-flight mass spectrometry. Analytical chemistry, 84(1), 403-411.

862 [33] Castro, B. M., Prieto, M., \& Silva, L. C. (2014). Ceramide: a simple sphingolipid with unique 863 biophysical properties. Progress in lipid research, 54, 53-67.

864 [34] Xu, Y. (2018). Lysophospholipid signaling in the epithelial ovarian cancer tumor 865 microenvironment. Cancers, 10(7), 227.

866 [35] Fujimoto, T., \& Parton, R. G. (2011). Not just fat: the structure and function of the lipid 867 droplet. Cold Spring Harbor perspectives in biology, 3(3), a004838.

868 [36] Welte, M. A. (2015). Expanding roles for lipid droplets. Current biology, 25(11), R470-R481. 
869 [37] Welte, M. A., \& Gould, A. P. (2017). Lipid droplet functions beyond energy storage.

870 Biochimica et Biophysica Acta (BBA)-Molecular and Cell Biology of Lipids, 1862(10), 1260-1272.

871 [38] Lydic, T. A., \& Goo, Y. H. (2018). Lipidomics unveils the complexity of the lipidome in 872 metabolic diseases. Clinical and translational medicine, 7(1), 4.

873 [39] Liebisch, G., Ekroos, K., Hermansson, M., \& Ejsing, C. S. (2017). Reporting of lipidomics 874 data should be standardized. Biochimica et Biophysica Acta (BBA)-Molecular and Cell Biology of 875 Lipids, 1862(8), 747-751.

876 [40] Baker, P. R. S., "Current State of Quantitation in Lipidomics Analysis", Avanti Polar lipids 877 blog, 23.03.2019. https://avantilipids.com/discussions/current-state-of-quantitation-in-lipidomics878 analysis

879 [41] Ivanova, P. T., Milne, S. B., Byrne, M. O., Xiang, Y., \& Brown, H. A. (2007).

880 Glycerophospholipid identification and quantitation by electrospray ionization mass spectrometry. 881 Methods in enzymology, 432, 21-57.

882 [42] Pi, J., Wu, X., \& Feng, Y. (2016). Fragmentation patterns of five types of phospholipids by 883 ultra-high-performance liquid chromatography electrospray ionization quadrupole time-of-flight 884 tandem mass spectrometry. Analytical Methods, 8(6), 1319-1332.

885 [43] Sullards, M. C., Liu, Y., Chen, Y., \& Merrill Jr, A. H. (2011). Analysis of mammalian 886 sphingolipids by liquid chromatography tandem mass spectrometry (LC-MS/MS) and tissue 887 imaging mass spectrometry (TIMS). Biochimica et Biophysica Acta (BBA)-Molecular and Cell 888 Biology of Lipids, 1811(11), 838-853.

889 [44] Khouri, Hania, "IM-QTOF Training”, Agilent Technologies, 12.-18.01.2019 at the Department 890 of Technical chemistry (Johannes-Kepler University Linz)

891 [45] Ferchaud-Roucher, V., Croyal, M., Moyon, T., Zair, Y., Krempf, M., \& Ouguerram, K. (2017). 892 Plasma lipidome analysis by liquid chromatography-high resolution mass spectrometry and ion 
893 mobility of hypertriglyceridemic patients on extended-release nicotinic acid: a pilot study. 894 Cardiovascular drugs and therapy, 31(3), 269-279.

895 [46] Lísa, M., Cífková, E., Khalikova, M., Ovčačíková, M., \& Holčapek, M. (2017). Lipidomic 896 analysis of biological samples: comparison of liquid chromatography, supercritical fluid 897 chromatography and direct infusion mass spectrometry methods. Journal of Chromatography A, $898 \quad 1525,96-108$.

899 [47] Blaženović, I., Shen, T., Mehta, S. S., Kind, T., Ji, J., Piparo, M., Cacciola, F., Mondello, L., \& 900 Fiehn, O. (2018). Increasing Compound Identification Rates in Untargeted Lipidomics Research 901 with Liquid Chromatography Drift Time-Ion Mobility Mass Spectrometry. Analytical chemistry, $90290(18), 10758-10764$.

903 [48] Hinz, C., Liggi, S., Mocciaro, G., Jung, S. M., Induruwa, I., Pereira, M. C., ... \& Fjeldsted, J. 904 C. (2019). A comprehensive UHPLC ion mobility QTOF method for profiling and quantification of 905 eicosanoids, other oxylipins and fatty acids. Analytical chemistry.

906 [49] Wenk, M. R. (2010). Lipidomics: new tools and applications. Cell, 143(6), 888-895.

907 [50] Van Meulebroek, L., De Paepe, E., Vercruysse, V., Pomian, B., Bos, S., Lapauw, B., \& 908 Vanhaecke, L. (2017). Holistic lipidomics of the human gut phenotype using validated ultra-high909 performance liquid chromatography coupled to hybrid orbitrap mass spectrometry. Analytical 910 chemistry, 89(22), 12502-12510.

911 [51] Rainville, P. D., Stumpf, C. L., Shockcor, J. P., Plumb, R. S., Nicholson, J. K. J. Proteome Res. $9126(2007) 552$.

913 [52] Drotleff, B., Hallschmid, M., \& Lämmerhofer, M. (2018). Quantification of steroid hormones 914 in plasma using a surrogate calibrant approach and UHPLC-ESI-QTOF-MS/MS with SWATH915 acquisition combined with untargeted profiling. Analytica chimica acta, 1022, 70-80. 
916 [53] Gobo, L. A., de Carvalho, L. M., Temp, F., Viana, C., \& Mello, C. F. (2018). A rapid method 917 for identification and quantification of prostaglandins in cerebral tissues by UHPLC-ESI-MS/MS 918 for the lipidomic in vivo studies. Analytical biochemistry, 545, 98-103.

919 [54] Capriotti, A. L., Cavaliere, C., \& Piovesana, S. (2019). Liposome protein corona 920 characterization as a new approach in nanomedicine. Analytical and bioanalytical chemistry, 1-14.

921 [55] Hyötyläinen, T., \& Orešič, M. (2015). Optimizing the lipidomics workflow for clinical 922 studies-practical considerations. Analytical and bioanalytical chemistry, 407(17), 4973-4993.

[56] Castro-Gómez, P., Montero, O., \& Fontecha, J. (2017). In-depth lipidomic analysis of 924 molecular species of triacylglycerides, diacylglycerides, glycerophospholipids, and sphingolipids of buttermilk by GC-MS/FID, HPLC-ELSD, and UPLC-QToF-MS. International journal of 926 molecular sciences, 18(3), 605.

[57] Lloyd, A. J., Beckmann, M., Wilson, T., Tailliart, K., Allaway, D., \& Draper, J. (2017). Ultra 928 high performance liquid chromatography-high resolution mass spectrometry plasma lipidomics can 930 distinguish between canine breeds despite uncontrolled environmental variability and nonstandardized diets. Metabolomics, 13(2), 15.

931 [58] Yuan, Z. X., Majchrzak-Hong, S., Keyes, G. S., Iadarola, M. J., Mannes, A. J., \& Ramsden, C. 932 E. (2018). Lipidomic profiling of targeted oxylipins with ultra-performance liquid chromatography933 tandem mass spectrometry. Analytical and bioanalytical chemistry, 410(23), 6009-6029.

934 [59] Gao, X., Luo, J., Lü, L., Zhang, L., Zhang, S., \& Cui, J. (2018). RNA-Seq and UHPLC-Q935 TOF/MS Based Lipidomics Study in Lysiphlebia japonica. Scientific reports, 8(1), 7802.

936 [60] Gupta, A., Dorlhiac, G. F., \& Streets, A. M. (2019). Quantitative imaging of lipid droplets in 937 single cells. Analyst, 144(3), 753-765. nanoflow liquid chromatography-tandem mass spectrometry. 938 Analytical chemistry, 89(4), 2488-2496.

939 [61] Drotleff, B., Illison, J., Schlotterbeck, J., Lukowski, R., \& Lämmerhofer, M. (2019). 940 Comprehensive Lipidomics of Mouse Plasma using Class-Specific Surrogate Calibrants and 
941 SWATH Acquisition for Large-Scale Lipid Quantification in Untargeted Analysis. Analytica 942 Chimica Acta.

943 [62] Zalloua, P., Kadar, H., Hariri, E., Farraj, L. A., Brial, F., Hedjazi, L., Lay, A. L., Colleum A., 944 Dubus, J., Touboul, D., Matsuda, F., Lathrop, M., Nicholson, J. K.,Dumas, M. E. \& Matsuda, F. 945 (2019). Untargeted Mass Spectrometry Lipidomics identifies correlation between serum 946 sphingomyelins and plasma cholesterol. Lipids in health and disease, 18(1), 38.

947 [63] Patterson, R. E., Kirpich, A. S., Koelmel, J. P., Kalavalapalli, S., Morse, A. M., Cusi, K., 948 Sunny, K., McIntyre, L. M., Garrett, T. J., \& Yost, R. A. (2017). Improved experimental data 949 processing for UHPLC-HRMS/MS lipidomics applied to nonalcoholic fatty liver disease. $950 \quad$ Metabolomics, 13(11), 142.

951 [64] Cheema, A., Mehta, K., Fatanmi, O., Wise, S., Hinzman, C., Wolff, J., \& Singh, V. (2017). A 952 Metabolomic and lipidomic serum signature from nonhuman primates administered with a 953 promising radiation countermeasure, gamma-tocotrienol. International journal of molecular 954 sciences, 19(1), 79.

955 [65] Liu, M. Y., Burgos, A., Ma, L., Zhang, Q., Tang, D., \& Ruan, J. (2017). Lipidomics analysis 956 unravels the effect of nitrogen fertilization on lipid metabolism in tea plant (Camellia sinensis L.). 957 BMC plant biology, 17(1), 165.

958 [66] Lee, J. W., Mok, H. J., Lee, D. Y., Park, S. C., Kim, G. S., Lee, S. E., Lee, Y.S., Kim, K. P., \& 959 Kim, H. D. (2017). UPLC-QqQ/MS-based lipidomics approach to characterize lipid alterations in 960 inflammatory macrophages. Journal of proteome research, 16(4), 1460-1469.

961 [67] Law, T. H., Volk, H. A., Pan, Y., Zanghi, B., \& Want, E. J. (2018). Metabolic perturbations 962 associated with the consumption of a ketogenic medium-chain TAG diet in dogs with idiopathic 963 epilepsy. British Journal of Nutrition, 120(5), 484-490. 
964 [68] He, Z., Wang, Y., Zhang, Y., Cheng, H., \& Liu, X. (2018). Stereoselective bioaccumulation of 965 chiral PCB 91 in earthworm and its metabolomic and lipidomic responses. Environmental pollution, $966238,421-430$.

967 [69] Chamberlain, C. A., Hatch, M., \& Garrett, T. J. (2019). Metabolomic and lipidomic 968 characterization of Oxalobacter formigenes strains HC1 and OxWR by UHPLC-HRMS. Analytical 969 and bioanalytical chemistry, 1-12.

970 [70] Jeucken, A., \& Brouwers, J. F. (2019). High-Throughput Screening of Lipidomic Adaptations 971 in Cultured Cells. Biomolecules, 9(2), 42.

972 [71] Creydt, M., Vuralhan-Eckert, J., Fromm, J., \& Fischer, M. (2019). Effects of elevated CO2 973 concentration on leaves and berries of black elder (Sambucus nigra) using UHPLC-ESI-QTOF974 MS/MS and gas exchange measurements. Journal of plant physiology, 234, 71-79.

975 [72] Wang, X., Xu, Y., Song, X., Jia, Q., Zhang, X., Qian, Y., \& Qiu, J. (2019). Analysis of 976 glycerophospholipid metabolism after exposure to PCB153 in PC12 cells through targeted 977 lipidomics by UHPLC-MS/MS. Ecotoxicology and environmental safety, 169, 120-127.

978 [73] Yang, J. S., Kim, J. Y., Lee, J. C., \& Moon, M. H. (2019). Investigation of lipidomic 979 perturbations in oxidatively stressed subcellular organelles and exosomes by asymmetrical flow 980 field-flow fractionation and nanoflow ultrahigh performance liquid chromatography-tandem mass 981 spectrometry. Analytica chimica acta, 1073, 79-89.

982 [74] Calderón, C., Rubarth, L., Cebo, M., Merfort, I., \& Lämmerhofer, M. (2019). Lipid Atlas of 983 Keratinocytes and Betulin Effects on its Lipidome Profiled by Comprehensive UHPLC- MS/MS 984 With Data Independent Acquisition Using Targeted Data Processing. Proteomics, 1900113.

985 [75] Kappler, L., Hoene, M., Hu, C., von Toerne, C., Li, J., Bleher, D., Hoffmann, C., Böhm, A., 986 Kollipara, L., Zischka, H., Königsrainer, A., Häring, H. U., Peter, A., Xu, G., Sickmann, A., 987 Hauck, S. M., Weigert, C., \& Lehmann, R. (2019). Linking bioenergetic function of mitochondria 
988 to tissue-specific molecular fingerprints. American Journal of Physiology-Endocrinology and 989 Metabolism.

990 [76] Fu, K., Wang, C., Gao, Y., Fan, S., Zhang, H., Sun, J., Jiang, Y., Liu, C., Guan, L., Liu, J., 991 Huang, M., \& Bi, H. (2019). Metabolomics and lipidomics reveal the effect of hepatic Vps33b 992 deficiency on bile acids and lipids metabolism. Frontiers in Pharmacology, 10, 276.

993 [77] Marqueño, A., Blanco, M., Maceda-Veiga, A., \& Porte, C. (2019). Skeletal muscle lipidomics 994 as a new tool to determine altered lipid homeostasis in fish exposed to urban and industrial 995 wastewaters. Environmental science \& technology.

996 [78] Hyötyläinen, T., \& Orešič, M. (2014). Systems biology strategies to study lipidomes in health 997 and disease. Progress in lipid research, 55, 43-60.

998 [79] Borowska-Strugiń, B., Druszczyńska, M., Lorkiewicz, W., Szewczyk, R., \& Ządzińska, E. 999 (2014). Mycolic acids as markers of osseous tuberculosis in the Neolithic skeleton from Kujawy 1000 region (central Poland). AnthropologicAl review, 77(2), 137-149.

1001 [80] Perrotti, F., Rosa, C., Cicalini, I., Sacchetta, P., Del Boccio, P., Genovesi, D., \& Pieragostino, 1002 D. (2016). Advances in lipidomics for cancer biomarkers discovery. International journal of 1003 molecular sciences, 17(12), 1992.

1004 [81] Crick, P. J., \& Guan, X. L. (2016). Lipid metabolism in mycobacteria—Insights using mass 1005 spectrometry-based lipidomics. Biochimica et Biophysica Acta (BBA)-Molecular and Cell Biology 1006 of Lipids, 1861(1), 60-67.

1007 [82] Daffé, M., \& Draper, P. (1997). The envelope layers of mycobacteria with reference to their 1008 pathogenicity. In Advances in microbial physiology (Vol. 39, pp. 131-203). Academic Press.

1009 [83] Zhang, Y., Liu, Y., Li, L., Wei, J., Xiong, S., \& Zhao, Z. (2016). High resolution mass 1010 spectrometry coupled with multivariate data analysis revealing plasma lipidomic alteration in 1011 ovarian cancer in Asian women. Talanta, 150, 88-96. 
1012 [84] Qadir, M. I., \& Malik, S. A. (2008). Plasma lipid profile in gynecologic cancers. European 1013 journal of gynaecological oncology, 29(2), 158.

1014 [85] Hu, C., van der Heijden, R., Wang, M., van der Greef, J., Hankemeier, T., \& Xu, G. (2009).

1015 Analytical strategies in lipidomics and applications in disease biomarker discovery. Journal of 1016 Chromatography B, 877(26), 2836-2846.

1017 [86] Zhao, Z., Xiao, Y., Elson, P., Tan, H., Plummer, S. J., Berk, M., ... \& Casey, G. (2007). Plasma 1018 lysophosphatidylcholine levels: potential biomarkers for colorectal cancer. Journal of clinical 1019 oncology, 25(19), 2696-2701.

1020 [87] Burgess, S., \& Harshfield, E. (2016). Mendelian randomization to assess causal effects of 1021 blood lipids on coronary heart disease: lessons from the past and applications to the future. Current 1022 opinion in endocrinology, diabetes, and obesity, 23(2), 124.

1023 [88] Holmes, M. V., Ala-Korpela, M., \& Smith, G. D. (2017). Mendelian randomization in 1024 cardiometabolic disease: challenges in evaluating causality. Nature Reviews Cardiology, 14(10), 1025577.

1026 [89] Dong, S., Zhang, R., Liang, Y., Shi, J., Li, J., Shang, F., Mao, X., \& Sun, J. (2017). Changes of 1027 myocardial lipidomics profiling in a rat model of diabetic cardiomyopathy using UPLC/Q-TOF/MS 1028 analysis. Diabetology \& metabolic syndrome, 9(1), 56.

1029 [90] Wu, J., Wu, Q., Wang, D., Kong, J., Dai, W., Wang, X., \& Yu, X. (2017). Common lipid 1030 features of lethal ventricular tarchyarrhythmias (LVTAs) induced by myocardial infarction and 1031 myocardial ion channel diseases. Scientific reports, 7(1), 4220.

1032 [91] Chen, Y., Wen, S., Jiang, M., Zhu, Y., Ding, L., Shi, H., Dong, P., Yang, J., \& Yang, Y. 1033 (2017). Atherosclerotic dyslipidemia revealed by plasma lipidomics on ApoE-/- mice fed a high1034 fat diet. Atherosclerosis, 262, 78-86.

1035 [92] Yan, B., Chu, H., Yang, D., Sze, K. H., Lai, P. M., Yuan, S., Shuai, H., Wang, Y., Kao, R. Y. 1036 T., Chan, J. F. W., \& Yuen, K. Y. (2019). Characterization of the Lipidomic Profile of Human 
1037 Coronavirus-Infected Cells: Implications for Lipid Metabolism Remodeling upon Coronavirus 1038 Replication. Viruses, 11(1), 73.

1039 [93] Tietz-Bogert, P., Kim, M., Cheung, A., Tabibian, J., Heimbach, J., Rosen, C., Nandakumar, 1040 M., Lazaridis, K. N., LaRusso, N. F., Sung, J. \& O’Hara, S. (2018). Metabolomic Profiling of 1041 Portal Blood and Bile Reveals Metabolic Signatures of Primary Sclerosing Cholangitis. 1042 International journal of molecular sciences, 19(10), 3188.

1043 [94] Wu, J., Wu, Q., Dai, W., Kong, J., Lv, J., Yu, X., Wang, X., \& Wang, D. (2018). Serum lipid 1044 feature and potential biomarkers of lethal ventricular tachyarrhythmia (LVTA) induced by 1045 myocardial ion channel diseases: a rat model study. International journal of legal medicine, 132(2), $1046 \quad 439-448$.

1047 [95] Chao, Y., Gao, S., Wang, X., Li, N., Zhao, H., Wen, X., Kou, Z., \& Dong, X. (2018). 1048 Untargeted lipidomics based on UPLC-QTOF-MS/MS and structural characterization reveals 1049 dramatic compositional changes in serum and renal lipids in mice with glyoxylate-induced 1050 nephrolithiasis. Journal of Chromatography B, 1095, 258-266.

1051 [96] Lee, J. H., Yang, J. S., Lee, S. H., \& Moon, M. H. (2018). Analysis of lipoprotein-specific 1052 lipids in patients with acute coronary syndrome by asymmetrical flow field-flow fractionation and 1053 nanoflow liquid chromatography-tandem mass spectrometry. Journal of Chromatography B, 1099, $105456-63$.

1055 [97] Kim, S. H., Yang, J. S., Lee, J. C., Lee, J. Y., Lee, J. Y., Kim, E., \& Moon, M. H. (2018). 1056 Lipidomic alterations in lipoproteins of patients with mild cognitive impairment and Alzheimer's 1057 disease by asymmetrical flow field-flow fractionation and nanoflow ultrahigh performance liquid 1058 chromatography-tandem mass spectrometry. Journal of Chromatography A, 1568, 91-100.

1059 [98] Cheema, A., Hinzman, C., Mehta, K., Hanlon, B., Garcia, M., Fatanmi, O., \& Singh, V. 1060 (2018). Plasma Derived Exosomal Biomarkers of Exposure to Ionizing Radiation in Nonhuman 1061 Primates. International journal of molecular sciences, 19(11), 3427. 
1062 [99] Shan, J., Qian, W., Shen, C., Lin, L., Xie, T., Peng, L., Xu, J., Yang, R., Ji, J., \& Zhao, X. 1063 (2018). High-resolution lipidomics reveals dysregulation of lipid metabolism in respiratory 1064 syncytial virus pneumonia mice. RSC Advances, 8(51), 29368-29377.

1065 [100] Lee, H., Choi, J. M., Cho, J. Y., Kim, T. E., Lee, H. J., \& Jung, B. H. (2018). Regulation of 1066 endogenic metabolites by rosuvastatin in hyperlipidemia patients: An integration of metabolomics 1067 and lipidomics. Chemistry and physics of lipids, 214, 69-83.

1068 [101] Wang, J., Zhang, L., Xiao, R., Li, Y., Liao, S., Zhang, Z., Yang, W., \& Liang, B. (2019). 1069 Plasma lipidomic signatures of spontaneous obese rhesus monkeys. Lipids in health and disease, $107018(1), 8$.

1071 [102] Lin, M., Wang, Z., Wang, D., Chen, X., \& Zhang, J. (2019). A mathematical model-assisted 1072 UHPLC-MS/MS method for global profiling and quantification of cholesteryl esters in 1073 hyperlipidemic golden hamsters. Analytical chemistry.

1074 [103] Shan, J., Qian, W., Kang, A., Peng, L., Xie, T., Lin, L., Di, L., Xiao, P., \& Zhou, W. (2019). 1075 Lipid profile perturbations in the plasma and lungs of mice with LPS-induced acute lung injury 1076 revealed by UHPLC-ESI-Q Exactive HF MS analysis. Journal of pharmaceutical and biomedical 1077 analysis, 162, 242-248.

1078 [104] Li, J., Gao, Y., Guan, L., Zhang, H., Chen, P., Gong, X., Li, D., Liang, X., Huang, M., \& Bi, 1079 H. (2019). Lipid Profiling of Peri-implantation Endometrium in Patients with Premature 1080 Progesterone Rise in the Late Follicular Phase. The Journal of Clinical Endocrinology \& 1081 Metabolism.

1082 [105] Gong, L. L., Yang, S., Zhang, W., Han, F. F., Lv, Y. L., Xuan, L. L., Liu, H., \& Liu, L. H. 1083 (2019). Discovery of Metabolite Profiles of Metabolic Syndrome Using Untargeted and Targeted 1084 LC-MS based Lipidomics Approach. Journal of pharmaceutical and biomedical analysis, 112848. 1085 [106] Yang, R., Zhang, Y., Qian, W., Peng, L., Lin, L., Xu, J., Xie, T., Ji, J., \& Shan, J. (2019). 1086 Surfactant Lipidomics of Alveolar Lavage Fluid in Mice Based on Ultra-High-Performance Liquid 
1087 Chromatography Coupled to Hybrid Quadrupole-Exactive Orbitrap Mass Spectrometry. 1088 Metabolites, 9(4), 80.

1089 [107] Li, Q., Liang, X., Zhao, L., Zhang, Z., Xue, X., Wang, K., \& Wu, L. (2017). UPLC-Q1090 exactive orbitrap/MS-based lipidomics approach to characterize lipid extracts from bee pollen and 1091 their in vitro anti-inflammatory properties. Journal of agricultural and food chemistry, 65(32), $1092 \quad 6848-6860$.

1093 [108] Zhang, H., Gao, Y., Sun, J., Fan, S., Yao, X., Ran, X., Zheng, C., Huang, M. \& Bi, H. (2017). 1094 Optimization of lipid extraction and analytical protocols for UHPLC-ESI-HRMS-based lipidomic 1095 analysis of adherent mammalian cancer cells. Analytical and bioanalytical chemistry, 409(22), $1096 \quad 5349-5358$

1097 [109] Chao, H. C., Chen, G. Y., Hsu, L. C., Liao, H. W., Yang, S. Y., Wang, S. Y., Li, Y. L., Tang, 1098 S. C., Tseng, Y. J., \& Kuo, C. H. (2017). Using precursor ion scan of 184 with liquid 1099 chromatography-electrospray ionization-tandem mass spectrometry for concentration normalization 1100 in cellular lipidomic studies. Analytica chimica acta, 971, 68-77.

1101 [110] Yang, J. S., Lee, J. C., Byeon, S. K., Rha, K. H., \& Moon, M. H. (2017). Size dependent 1102 lipidomic analysis of urinary exosomes from patients with prostate cancer by flow field-flow 1103 fractionation and nanoflow liquid chromatography-tandem mass spectrometry. Analytical 1104 chemistry, 89(4), 2488-2496.

1105 [111] Li, Z., Guan, M., Lin, Y., Cui, X., Zhang, Y., Zhao, Z., \& Zhu, J. (2017). Aberrant lipid 1106 metabolism in hepatocellular carcinoma revealed by liver lipidomics. International journal of 1107 molecular sciences, 18(12), 2550.

1108 [112] Lee, G. B., Lee, J. C., \& Moon, M. H. (2019). Plasma lipid profile comparison of five 1109 different cancers by nanoflow ultrahigh performance liquid chromatography-tandem mass 1110 spectrometry. Analytica Chimica Acta. 
1111 [113] Chen, Y., Ma, Z., Shen, X., Li, L., Zhong, J., Min, L. S., Xu, L., Li, H., Zhang, J., \& Dai, L.

1112 (2018). Serum lipidomics profiling to identify biomarkers for non-small cell lung cancer. BioMed 1113 research international, 2018.

1114 [114] Jurowski, K., Kochan, K., Walczak, J., Barańska, M., Piekoszewski, W., \& Buszewski, B. 1115 (2017). Comprehensive review of trends and analytical strategies applied for biological samples 1116 preparation and storage in modern medical lipidomics: State of the art. TrAC Trends in Analytical 1117 Chemistry, 86, 276-289.

1118 [115] Patterson, R. E., Ducrocq, A. J., McDougall, D. J., Garrett, T. J., \& Yost, R. A. (2015). 1119 Comparison of blood plasma sample preparation methods for combined LC-MS lipidomics and 1120 metabolomics. Journal of Chromatography B, 1002, 260-266.

1121 [116] Tumanov, S., \& Kamphorst, J. J. (2017). Recent advances in expanding the coverage of the 1122 lipidome. Current opinion in biotechnology, 43, 127-133.

1123 [117] López-Bascón, M. A., Calderón-Santiago, M., Sánchez-Ceinos, J., Fernández-Vega, A.,

1124 Guzmán-Ruiz, R., López-Miranda, J., ... \& Priego-Capote, F. (2018). Influence of sample

1125 preparation on lipidomics analysis of polar lipids in adipose tissue. Talanta, 177, 86-93.

1126 [118] Rudralingam, Velauthan et al. "Ascites matters" Ultrasound (Leeds, England) vol. 25,2 1127 (2016): 69-79.

1128 [119] Folch, J., Lees, M., \& Sloane Stanley, G. H. (1957). A simple method for the isolation and 1129 purification of total lipides from animal tissues. J biol Chem, 226(1), 497-509.

1130 [120] Löfgren, L., Ståhlman, M., Forsberg, G. B., Saarinen, S., Nilsson, R., \& Hansson, G. I. 1131 (2012). The BUME method: a novel automated chloroform-free 96-well total lipid extraction 1132 method for blood plasma. Journal of lipid research, 53(8), 1690-1700.

1133 [121] Lofgren, L., Stahlman, M., Forsberg, G. B., Saarinen, S., Nilsson, R., \& Hansson, G. I. 1134 (2012). The BUME method: a novel automated chloroform-free 96-well total lipid extraction 1135 method for blood plasma. Journal of lipid research, jlr-D023036. 
1136 [122] Drotleff, B., \& Lämmerhofer, M. (2019). Guidelines for Selection of Internal Standard-based 1137 Normalization Strategies in Untargeted Lipidomic Profiling by LC-HR-MS/MS. Analytical 1138 chemistry.

1139 [123] Reis, A., Rudnitskaya et al. 2013 A comparison of five lipid extraction solvent systems for 1140 lipidomic studies of human LDL. J Lipid Res. 2013 Jul; 54(7): 1812-1824.

1141 [124] Cruz, M., Wang, M., Frisch- Daiello, J., \& Han, X. (2016). Improved Butanol-Methanol 1142 (BUME) Method by Replacing Acetic Acid for Lipid Extraction of Biological Samples. Lipids, $1143 \quad 51(7), 887-896$.

1144 [125] Teo, C. C., Chong, W. P. K., Tan, E., Basri, N. B., Low, Z. J., \& Ho, Y. S. (2015). Advances 1145 in sample preparation and analytical techniques for lipidomics study of clinical samples. TrAC 1146 Trends in Analytical Chemistry, 66, 1-18.

1147 [126] Pulfer, M., \& Murphy, R. C. (2003). Electrospray mass spectrometry of phospholipids. Mass 1148 spectrometry reviews, 22(5), 332-364.

1149 [127] Bang, G., Kim, Y. H., Yoon, J., Yu, Y. J., Chung, S., \& Kim, J. A. (2017). On-Chip Lipid 1150 Extraction Using Superabsorbent Polymers for Mass Spectrometry. Analytical chemistry, 89(24), $1151 \quad 13365-13373$.

1152 [128] Onorato, J. M., Shipkova, P., Minnich, A., Aubry, A. F., Easter, J., \& Tymiak, A. (2014). 1153 Challenges in accurate quantitation of lysophosphatidic acids in human biofluids. Journal of lipid 1154 research, 55(8), 1784-1796. 1194/jlr.D050070

1155 [129] Bligh, E. G., \& Dyer, W. J. (1959). A rapid method of total lipid extraction and purification. 1156 Canadian journal of biochemistry and physiology, 37(8), 911-917

1157 [130] Calderón, C., Sanwald, C., Schlotterbeck, J., Drotleff, B., \& Lämmerhofer, M. (2019). 1158 Comparison of simple monophasic versus classical biphasic extraction protocols for comprehensive 1159 UHPLC-MS/MS lipidomic analysis of Hela cells. Analytica chimica acta, 1048, 66-74. 
1160 [131] MacNair, J. E., Lewis, K. C., \& Jorgenson, J. W. (1997). Ultrahigh-pressure reversed-phase

1161 liquid chromatography in packed capillary columns. Analytical chemistry, 69(6), 983-989.

1162 [132] Avela, H. F., Sirén, H., (2019), Analytical tools and current statistical methods used in mass

1163 spectrometry of glycero-, glycerophospho- and sphingolipids. A review on liquid chromatographic

1164 measurements on-line coupled with various methods of mass spectrometry, in-print

1165 [133] Krautbauer, S., Büchler, C., \& Liebisch, G. (2016). Relevance in the use of appropriate

1166 internal standards for accurate quantification using LC-MS/MS: tauro-conjugated bile acids as an 1167 example. Analytical chemistry, 88(22), 10957-10961.

1168 [134] Liebisch, G., The Role of LC-MS in Lipidomics. LCGC Europe, 2017, 30 240-242

1169 [135] Kartsova, L. A., Bessonova, E. A., \& Somova, V. D. (2019). Hydrophilic Interaction

1170 Chromatography. Journal of Analytical Chemistry, 74(5), 415-424.

1171 [136] Erngren, I., Haglöf, J., Engskog, M. K., Nestor, M., Hedeland, M., Arvidsson, T., \& 1172 Pettersson, C. (2019). Adduct formation in electrospray ionisation-mass spectrometry with 1173 hydrophilic interaction liquid chromatography is strongly affected by the inorganic ion 1174 concentration of the samples. Journal of Chromatography A, 1600, 174-182.

1175 [137] Lin, L., Yu, Q., Yan, X., Hang, W., Zheng, J., Xing, J., \& Huang, B. (2010). Direct infusion mass spectrometry or liquid chromatography mass spectrometry for human metabonomics? A

1177 serum metabonomic study of kidney cancer. Analyst, 135(11), 2970-2978.

1178 [138] Fekete, S., Schappler, J., Veuthey, J. L., \& Guillarme, D. (2014). Current and future trends in 1179 UHPLC. TrAC Trends in Analytical Chemistry, 63, 2-13.

$1180 \quad[139]$

1181 [140] Danne-Rasche, N., Coman, C., \& Ahrends, R. (2018). Nano-LC/NSI MS refines lipidomics 1182 by enhancing lipid coverage, measurement sensitivity, and linear dynamic range. Analytical 1183 chemistry, 90(13), 8093-8101. 
1184 [141] Jeucken, A., Molenaar, M. R., van de Lest, C. H., Jansen, J. W., Helms, J. B., \& Brouwers, J. 1185 F. (2019). A comprehensive functional characterization of Escherichia coli lipid genes. Cell reports, 27(5), 1597-1606

[142] Han, X., \& Gross, R. W. (1994). Electrospray ionization mass spectroscopic analysis of 1188 human erythrocyte plasma membrane phospholipids. Proceedings of the National Academy of 1189 Sciences, 91(22), 10635-10639.

1190 [143] Holcapek, M., Liebisch, G., \& Ekroos, K. (2018). Lipidomic Analysis.

1191 [144] Yang, K., \& Han, X. (2016). Lipidomics: techniques, applications, and outcomes related to 1192 biomedical sciences. Trends in biochemical sciences, 41(11), 954-969.

1193 [145] Kohlwein, S. D. (2017). Opinion articles on lipidomics-A critical assessment of the state-of1194 the-art. Biochimica et biophysica acta. Molecular and cell biology of lipids, 1862(8), 729.

1195 [146] Sethi, S., \& Brietzke, E. (2017). Recent advances in lipidomics: Analytical and clinical 1196 perspectives. Prostaglandins \& other lipid mediators, 128, 8-16.

1197

[147] Calderón, C., Sanwald, C., Schlotterbeck, J., Drotleff, B., \& Lämmerhofer, M. (2019). Comparison of simple monophasic versus classical biphasic extraction protocols for comprehensive UHPLC-MS/MS lipidomic analysis of Hela cells. Analytica chimica acta, 1048, 66-74.

[148] Zhang, J., Rector, J., Lin, J. Q., Young, J. H., Sans, M., Katta, N., Giese, N., Yu, W., Nagi, C., Suliburk, J., Liu, J., Bensussan, A.m DeHoog, R. J., Garza, K. Y., Ludolph, B., Sorace, A. G.,, Syed, A., Zahedivash, A., Milner, T. E., \& Eberlin, L. S. (2017). Nondestructive tissue analysis for ex vivo and in vivo cancer diagnosis using a handheld mass spectrometry system. Science translational medicine, 9(406), eaan3968.

[149] Phelps, D. L., Balog, J., Gildea, L. F., Bodai, Z., Savage, A., El-Bahrawy, M. A., Speller, A. V. M., Rosini, F., Kudo, H., McKenzie, J. S., Brown, R., Takáts, Z., \& Brown, R. (2018). The surgical intelligent knife distinguishes normal, borderline and malignant gynaecological tissues 
1208 using rapid evaporative ionisation mass spectrometry (REIMS). British journal of cancer, 118(10), 12091349.

1210 [150] Avela, H. F. (2019). Recent Developments and Challenges in Chromatographic Lipidomics. 1211 Helsinki University, E-thesis

1212 [151] Lorenzen, W., Bozhüyük, K. A., Cortina, N. S., \& Bode, H. B. (2014). A comprehensive 1213 insight into the lipid composition of Myxococcus xanthus by UPLC-ESI-MS. Journal of lipid 1214 research, 55(12), 2620-2633.

1215 [152] Cajka, T., \& Fiehn, O. (2014). Comprehensive analysis of lipids in biological systems by 1216 liquid chromatography-mass spectrometry. TrAC Trends in Analytical Chemistry, 61, 192-206.

1217 [153] Merrill Jr, A. H., Sullards, M. C., Allegood, J. C., Kelly, S., \& Wang, E. (2005). 1218 Sphingolipidomics: high-throughput, structure-specific, and quantitative analysis of sphingolipids 1219 by liquid chromatography tandem mass spectrometry. Methods, 36(2), 207-224.

1220 [154] Lemke, A., Castillo-Sánchez, J. C., Prodinger, F., Ceranic, A., Hennerbichler-Lugscheider, S., 1221 Pérez-Gil, J., Redl, H. \& Wolbank, S. (2017). Human amniotic membrane as newly identified 1222 source of amniotic fluid pulmonary surfactant. Scientific reports, 7(1), 6406.

1223 [155] Xuan, Q., Hu, C., Yu, D., Wang, L., Zhou, Y., Zhao, X., Li, Q, Hou, X. \& Xu, G. (2018). 1224 Development of a High Coverage Pseudotargeted Lipidomics Method Based on Ultra-High 1225 Performance Liquid Chromatography-Mass Spectrometry. Analytical chemistry, 90(12), 760812267616.

1227 [156] De Paepe, E., Van Meulebroek, L., Rombouts, C., Huysman, S., Verplanken, K., Lapauw, B., 1228 Wauters, J., Hemeryck, L. Y., Vanhaecke, L., \& Vanhaecke, L. (2018). A validated multi-matrix 1229 platform for metabolomic fingerprinting of human urine, feces and plasma using ultra-high 1230 performance liquid-chromatography coupled to hybrid orbitrap high-resolution mass spectrometry. 1231 Analytica chimica acta, 1033, 108-118. 
1232 [157] Ulmer, C. Z., Jones, C. M., Yost, R. A., Garrett, T. J., \& Bowden, J. A. (2018). Optimization 1233 of Folch, Bligh-Dyer, and Matyash sample-to-extraction solvent ratios for human plasma-based 1234 lipidomics studies. Analytica chimica acta, 1037, 351-357.

1235 [158] Byeon, S. K., Kim, J. Y., Lee, J. Y., Chung, B. C., Seo, H. S., \& Moon, M. H. (2015). Top1236 down and bottom-up lipidomic analysis of rabbit lipoproteins under different metabolic conditions 1237 using flow field-flow fractionation, nanoflow liquid chromatography and mass spectrometry. 1238 Journal of Chromatography A, 1405, 140-148.

1239 [159] Chang, W. Q., Zhou, J. L., Li, Y., Shi, Z. Q., Wang, L., Yang, J., Li, P., Liu, L. F., \& Xin, G. 1240 Z. (2017). An in vitro approach for lipolysis measurement using high-resolution mass spectrometry 1241 and partial least squares based analysis. Analytica chimica acta, 950, 138-146.

1242 [160] Triebl, A., Trötzmüller, M., Hartler, J., Stojakovic, T., \& Köfeler, H. C. (2017). Lipidomics 1243 by ultrahigh performance liquid chromatography-high resolution mass spectrometry and its 1244 application to complex biological samples. Journal of Chromatography B, 1053, 72-80.

1245 [161] Witting, M., Ruttkies, C., Neumann, S., \& Schmitt-Kopplin, P. (2017). LipidFrag: Improving 1246 reliability of in silico fragmentation of lipids and application to the Caenorhabditis elegans 1247 lipidome. PloS one, 12(3), e0172311.

1248 [162] Ejsing, C. S. (2007). Molecular characterization of the lipidome by mass spectrometry 1249 (Doctoral dissertation, Verlag nicht ermittelbar).

1250 [163] Blaas, N., \& Humpf, H. U. (2013). Structural profiling and quantitation of glycosyl inositol 1251 phosphoceramides in plants with Fourier transform mass spectrometry. Journal of agricultural and 1252 food chemistry, 61(18), 4257-4269.

1253 [164] S Elkhayat, E., A Mohamed, G., \& RM Ibrahim, S. (2012). Activity and structure elucidation 1254 of ceramides. Current Bioactive Compounds, 8(4), 370-409. 
1255 [165] Scherer M., Schmitz G., Liebisch G. 2010. Simultaneous quantification of cardiolipin, 1256 bis(monoacylglycero)phosphate and their precursors by hydrophilic interaction LC-MS/MS 1257 including correction of isotopic overlap. Anal. Chem. 82: 8794-8799.

1258 [166] Berry, Karin A. Zemski, and Robert C. Murphy. "Electrospray ionization tandem mass 1259 spectrometry of glycerophosphoethanolamine plasmalogen phospholipids." Journal of the American 1260 Society for Mass Spectrometry 15.10 (2004): 1499-1508.

1261 [167] Hsu, F. F., Turk, J., Rhoades, E. R., Russell, D. G., Shi, Y., \& Groisman, E. A. (2005). 1262 Structural characterization of cardiolipin by tandem quadrupole and multiple-stage quadrupole ion1263 trap mass spectrometry with electrospray ionization. Journal of the American Society for Mass 1264 Spectrometry, 16(4), 491-504.

1265 [168] Smith, P. B., Snyder, A. P., \& Harden, C. S. (1995). Characterization of bacterial 1266 phospholipids by electrospray ionization tandem mass spectrometry. Analytical chemistry, 67(11), $1267 \quad 1824-1830$.

1268 [169] Ring, M. W., Schwär, G., \& Bode, H. B. (2009). Biosynthesis of 2- hydroxy and iso- even 1269 fatty acids is connected to sphingolipid formation in myxobacteria. ChemBioChem, 10(12), 200312702010.

1271 [170] Curtis, P. D., Geyer, R., White, D. C., \& Shimkets, L. J. (2006). Novel lipids in Myxococcus 1272 xanthus and their role in chemotaxis. Environmental microbiology, 8(11), 1935-1949.

1273 [171] Kleinig, H. (1972). Membranes from Myxococcus fulvus (myxobacterales) containing 1274 carotenoid glucosides: I. Isolation and composition. Biochimica et Biophysica Acta (BBA)1275 Biomembranes, 274(2), 489-498.

1276 [172] Orndorff, P. E., \& Dworkin, M. (1980). Separation and properties of the cytoplasmic and 1277 outer membranes of vegetative cells of Myxococcus xanthus. Journal of bacteriology, 141(2), 9141278927. 
1279 [173] Murphy, R. C., James, P. F., McAnoy, A. M., Krank, J., Duchoslav, E., \& Barkley, R. M.

1280 (2007). Detection of the abundance of diacylglycerol and triacylglycerol molecular species in cells

1281 using neutral loss mass spectrometry. Analytical biochemistry, 366(1), 59-70.

1282 [174] Rampler, E., Coman, C., Hermann, G., Sickmann, A., Ahrends, R., \& Koellensperger, G. 1283 (2017). LILY-lipidome isotope labeling of yeast: in vivo synthesis of 13 C labeled reference lipids 1284 for quantification by mass spectrometry. Analyst, 142(11), 1891-1899.

1285 [175] Wenk, M. R. (2005). The emerging field of lipidomics. Nature reviews Drug discovery, 4(7), 1286594.

1287 [176] Blanksby, S. J., \& Mitchell, T. W. (2010). Advances in mass spectrometry for lipidomics. 1288 Annual Review of Analytical Chemistry, 3, 433-465.

1289 [177] Liebisch, G., Vizcaíno, J. A., Köfeler, H., Trötzmüller, M., Griffiths, W. J., Schmitz, G., ... \& 1290 Wakelam, M. J. (2013). Shorthand notation for lipid structures derived from mass spectrometry. 1291 Journal of lipid research, 54(6), 1523-1530.

1292 [178] Pham, T. H., Zaeem, M., Fillier, T. A., Nadeem, M., Vidal, N. P., Manful, C., Cheema, S., 1293 Cheema, M., \& Thomas, R. H. (2019). Targeting Modified Lipids during Routine Lipidomics 1294 Analysis using HILIC and C30 Reverse Phase Liquid Chromatography coupled to Mass 1295 Spectrometry. Scientific reports, 9(1), 5048.

1296 [179] Gorrochategui, E., Jaumot, J., Lacorte, S., \& Tauler, R. (2016). Data analysis strategies for 1297 targeted and untargeted LC-MS metabolomic studies: Overview and workflow. TrAC Trends in 1298 Analytical Chemistry, 82, 425-442.

1299 [180] Benjamini, Y., \& Hochberg, Y. (1995). Controlling the false discovery rate: a practical and 1300 powerful approach to multiple testing. Journal of the Royal statistical society: series B 1301 (Methodological), 57(1), 289-300. 
1302 [181] Fu, K., Wang, C., Gao, Y., Fan, S., Zhang, H., Sun, J., Jiang, Y., Liu, J., Huang, M., \& Bi, H. 1303 (2019). Metabolomics and lipidomics reveal the effect of hepatic Vps33b deficiency on bile acids 1304 and lipids metabolism. Frontiers in Pharmacology, 10, 276.

1305 [182] Storey, J. D. (2003). The positive false discovery rate: a Bayesian interpretation and the q1306 value. The Annals of Statistics, 31(6), 2013-2035.

1307 [183] Nachar, N. (2008). The Mann-Whitney U: A test for assessing whether two independent 1308 samples come from the same distribution. Tutorials in quantitative Methods for Psychology, 4(1), $1309 \quad 13-20$.

1310 [184] Koelmel, J. P., Kroeger, N. M., Ulmer, C. Z., Bowden, J. A., Patterson, R. E., Cochran, J. A., 1311 Beecher, C. W. W., Garrett, T. J.\& Yost, R. A. (2017). LipidMatch: an automated workflow for 1312 rule-based lipid identification using untargeted high-resolution tandem mass spectrometry data. $1313 \quad$ BMC bioinformatics, 18(1), 331.

1314 [185] Kind, T., Liu, K. H., Lee, D. Y., DeFelice, B., Meissen, J. K., \& Fiehn, O. (2013). LipidBlast 1315 in silico tandem mass spectrometry database for lipid identification. Nature methods, 10(8), 755. 1316 [186] Lin, L., Yu, Q., Yan, X., Hang, W., Zheng, J., Xing, J., \& Huang, B. (2010). Direct infusion 1317 mass spectrometry or liquid chromatography mass spectrometry for human metabonomics? A 1318 serum metabonomic study of kidney cancer. Analyst, 135(11), 2970-2978.

1319 [187] Smith, R., Mathis, A. D., Ventura, D., \& Prince, J. T. (2014). Proteomics, lipidomics, 1320 metabolomics: a mass spectrometry tutorial from a computer scientist's point of view. BMC 1321 bioinformatics, 15(7), S9.

1322 [188] Griffiths, W. J., \& Wang, Y. (2017). Sterolomics: State of the art, developments, limitations 1323 and challenges. Biochimica et Biophysica Acta (BBA)-Molecular and Cell Biology of Lipids, $1324 \quad 1862(8), 771-773$.

1325 [189] Beccaria, M., Inferrera, V., Rigano, F., Gorynski, K., Purcaro, G., Pawliszyn, J., Dugo, P., \& 1326 Mondello, L. (2017). Highly informative multiclass profiling of lipids by ultra-high performance 
1327 liquid chromatography-Low resolution (quadrupole) mass spectrometry by using electrospray 1328 ionization and atmospheric pressure chemical ionization interfaces. Journal of Chromatography A, $13291509,69-82$.

1330 [190] Kim, H. S., Waqued, S. C., Nodurft, D. T., Devarenne, T. P., Yakovlev, V. V., \& Han, A. 1331 (2017). Raman spectroscopy compatible PDMS droplet microfluidic culture and analysis platform 1332 towards on-chip lipidomics. Analyst, 142(7), 1054-1060.

\section{List of Figures}

1334 Figure 1. Fatty acid elongation and desaturation processes by a de novo synthesis and b dietary 1335 uptake. The n-families are determined by the position of the double bond. Ref. [16]

1336 Figure 2. FAs, [12] GL and GP [16] molecular characteristics. Acyl carnitine (AcCA) and 1337 cardiolipin (CL) structures are depicted to remind of more complex and less understood lipid 1338 structures.

1339 Figure 3. Typical sphingolipid (SP) structures. R1 is an amine that usually has a fatty acid linked to 1340 it with a nitrogen bond. R2 on the other hand is either a free hydroxyl group as in ceramides but 1341 occupied with a characteristic phosporylated molecule in sphingomyelins (SMs). Structures derived 1342 from ref. [17].

1343 Figure 4. Fragmentation of three typical GPs as reported by Ivanova et al. [41] Naturally, the exact 1344 mass should be used for the proper identification of a "lipid class-selective fragment" [39]. Also, 1345 the de-/protonated state of the ionized lipid species and neutral losses should be considered.

1346 Figure 5. Fragmentation patterns of SPs with denoted sites of cleavage (for S and Cer) [153]. Note 1347 that SA fragments are nearly identical with sphingosine species, except they have two more 1348 hydrogen and no DB in the backbone $\left(\mathrm{M}_{\mathrm{SA}}=\mathrm{M}_{\mathrm{S}}+2\right)$. Ref. [43]

1349 Figure 6. Combined base peak chromatogram and contour plot of HeLa lipids during exponential 1350 cell growth. Cells were lysed in chloroform / methanol and the resulting extract was injected 1351 directly on the high-performance liquid chromatography (HPLC) column after removal of 1352 precipitated proteins and macro-molecules by centrifugation (REF [70]) 
1353 Figure 7. Structures of (a) nonpolar and (b) polar lipid subclass in separation techniques. Reprinted 1354 with permission from ref [116], DOI: 0958-1669/ 2016. S Tumanov and JJ Kamphorst. Published 1355 by Elsevier Ltd, an open access article under the CC BY license.

1356 Figure 8. Product ion scans of negative mode HILIC-separated lipids from a standard mix 1357 (molecular ion is marked with an arrow). Reprinted from ref [178] @ 2019, Springer Nature 1358 MMPE: monomethyl-phosphatidylethanolamine, DMPE: dimethyl-phosphatidylethanolamine 1359 Figure 9. Creation, validation, and application of in-silico generated MS/MS spectra in LipidBlast. 1360 (a) New lipid compound structures were generated using in-silico methods. Lipid core structure 1361 scaffolds were connected via a linker to fatty acyls with different chain lengths and different 1362 degrees of unsaturation. Asterisks denote connection points. (b) Reference tandem spectra (top) 1363 were used to simulate mass spectral fragmentations and ion abundances of the in-silico spectra 1364 (bottom). The compound shown is $\mathrm{PC}(16: 0 / 16: 1)$ at precursor $m / z 732.55[\mathrm{M}+\mathrm{H}]^{+}$. (c) For lipid 1365 identification, MS/MS spectra obtained from LC-MS/MS or direct-infusion experiments were 1366 submitted to LipidBlast. Ref. [185]

1367 Figure 10. LipidBlast was mostly developed with ion trap tandem mass spectra but can be used 1368 with data from other platforms such as QTOF mass spectrometers. a) The Cardiolipin example 1369 shows that even in the in the case of the non-matching but abundant precursor ion at $\mathrm{m} / \mathrm{z}, 1239.8355$ $1370[\mathrm{M}-\mathrm{H}]^{-}$, the correct result is obtained with LipidBlast. b) The standard reference compound with 1371 precursor $m / z=793.4841[\mathrm{M}-\mathrm{H}]^{-}$is correctly identified as phosphatidylinositol $\mathrm{PI}(17: 0 / 14: 1)$ as first 1372 hit in a library search with LipidBlast. Ref. [185]

1375 List of Tables

1376 Table 1. Research on homeostatic profiling (10 articles)

1377 Table 2. Metabolomics research topics. (16 articles) 
1378 Table 3. UHPLC-research on lipidomic pathway-based diseases and potential biomarkers

1379 (19 articles)

1380 Table 4. Lipidomic UHPLC-research focusing on cancer

1381 Table 5. Extraction and sample purification methods used in lipidomics.

1382 Table 6. Molecular species formed during electrospray ionization of lipids. Ref. [152]

1383 Table 7. Classical biological material types. Extension to Jurowski et al.’s Table (Ref. [114]).

1384 Table 8. Alternative bioloical materials. Extension to Supplementary Table 1 by Jurovski et al.

1385 [114] Hex is an abbreviation for hexosyl, Hex2 for dihexosyl

1386 Table 9. Cell culture matrices analyzed with UHPLC-MS techniques.

1387 Hex is an abbreviation for hexosyl, Hex2 for dihexosyl

1388 Table 10. Common lipids according to research topic and identification approach. When 1389 "Identified lipids" are indicated as a sum without any explanation, it means that the first number is 1390 the amount of lipids found in positive and the second in negative mode (positive+negative).

1391 Table 11. Extension of lipid classes analyzed in different research articles (2017-2019) 
Table 1. Research on homeostatic profiling (10 articles)

\begin{tabular}{|c|r|l|l|}
\hline Year & Citation & Theme & Subject \\
\hline 2017 & {$[50]$} & Homeostatic profiling & Human gut phenotype profiling \\
\hline 2017 & {$[56]$} & Homeostatic profiling & Buttermilk profiling \\
\hline 2017 & {$[57]$} & Homeostatic profiling & Distinguishing between canine breeds \\
\hline 2018 & {$[58]$} & Homeostatic profiling & Oxylipin analysis in human patients \\
\hline 2018 & {$[15]$} & Homeostatic profiling & SM and Cer in multiple tissues/cell cultures of humans, dogs, mice and rats \\
\hline 2018 & {$[59]$} & Homeostatic profiling & Larvae profiling (Lysphlebia japonica) \\
\hline 2018 & {$[52]$} & Homeostatic profiling & Steroid hormone quantification in human plasma \\
\hline 2018 & {$[53]$} & Homeostatic profiling & In vivo prostaglandin identification and quantitation in human brain tissue \\
\hline 2019 & {$[60]$} & Homeostatic profiling & Coherent Raman scattering (CRS), non-destructive lipid/metabolite profiling \\
\hline 2019 & {$[61]$} & Homeostatic profiling & Mouse plasma analysis with matrix-matched calibrants and SWATH* MS/MS \\
\hline
\end{tabular}

*SWATH: sequential window acquisition of all theoretical fragment ion mass spectra 
Table 2. Metabolomics research topics. (16 articles)

\begin{tabular}{|c|c|c|c|}
\hline Year & Citation & Theme & Subject \\
\hline 2017 & [63] & $\begin{array}{l}\text { Applications / } \\
\text { Metabolomics }\end{array}$ & Nonalcoholic fatty liver disease profiling, improved data-analysis \\
\hline 2017 & [64] & Metabolomics & Radiation countermeasure mechanism, GT3 inhibitor test \\
\hline 2017 & [65] & Metabolomics & Fertilization of plants \\
\hline 2017 & [66] & Metabolomics & Inflammatory macrophage characterization \\
\hline 2018 & [67] & Metabolomics & Ketogenic diet, consequent metabolic perturbations, odd carbon lipids \\
\hline 2018 & {$[68]$} & Metabolomics & Bioaccumulation \& metabolomic response to chiral PCB 91 \\
\hline 2019 & {$[62]$} & Metabolomics & Correlation between serum SMs and plasma cholesterol \\
\hline 2019 & [69] & Metabolomics & Oxalobacter profiling for oxalate-based disease research \\
\hline 2019 & [70] & Metabolomics & High-throughput 96-well cell culture assay \\
\hline 2019 & [71] & Metabolomics & Elevated $\mathrm{CO} 2$ concentration, leaves \& berries of the black elder plant \\
\hline 2019 & [72] & Metabolomics & Lipido-metabolic changes upon PCB153/PC12 exposure \\
\hline 2019 & [73] & Metabolomics & Lipidomic perturbations of HEK 293 cells and exosomes under oxidative stress \\
\hline 2019 & [74] & Metabolomics & Keratinocyte lipidomics affected by botulin \\
\hline 2019 & [75] & Metabolomics & Analysis on the cellular functions of liver and skeletal muscle mitochondria \\
\hline 2019 & [76] & Metabolomics & Targeted bile acid analysis for validation of liver cholestasis-associated protein \\
\hline 2019 & [87] & Metabolomics & Skeletal muscle analysis of urban industrial waste water-exposed fish* \\
\hline
\end{tabular}

*B. meridionalis and S. laietanus 
Table 3. UHPLC-research on lipidomic pathway-based diseases and potential biomarkers (19 articles)

\begin{tabular}{|c|c|c|c|}
\hline Year & Citation & Theme & Subject \\
\hline 2017 & {$[89]$} & Diseases and Biomarkers & Diabetic cardiomyopathy \\
\hline 2017 & {$[90]$} & Diseases and Biomarkers & $\begin{array}{l}\text { Lethal ventricular tachyarrhytmia induced by myocardial ion channel } \\
\text { diseases \& infarction }\end{array}$ \\
\hline 2017 & [91] & Diseases and Biomarkers & Atheroslerotic dyslipidemia via a high-fat diet on mice \\
\hline 2018 & {$[92]$} & Diseases and Biomarkers & In vitro Coronavirus-infection of cell cultures \\
\hline 2018 & [93] & Diseases and Biomarkers & Primary sclerosing cholangitis \\
\hline 2018 & [94] & Diseases and Biomarkers & $\begin{array}{l}\text { Lethal ventricular tachyarrhytmia induced by myocardial ion channel } \\
\text { diseases }\end{array}$ \\
\hline 2018 & [95] & Diseases and Biomarkers & Glyoxylate-induced nephrolithiasis \\
\hline 2018 & [96] & Diseases and Biomarkers & Acute coronary syndrome \\
\hline 2018 & [97] & Diseases and Biomarkers & Alzheimer's disease and amnestic mild cognitive impairment \\
\hline 2018 & [98] & Diseases and Biomarkers & Plasma derived exosomal biomarkers, radiation exposure \\
\hline 2018 & [99] & Diseases and Biomarkers & Dysregulation in respiratory syncytial virus pneumonia (mouse) \\
\hline 2018 & {$[100]$} & Diseases and Biomarkers & Regulation of rosuvastatin in lipidemia patients \\
\hline 2019 & {$[101]$} & Diseases and Biomarkers & Obesity biomarkers (rhesus monkey) \\
\hline 2019 & {$[102]$} & Diseases and Biomarkers & $\begin{array}{l}\text { VLDL, LDL and HDL CEs during lipidemia (golden hamster), } \\
\text { unsaturation correlation with logarithmic mathematical model }\end{array}$ \\
\hline 2019 & {$[103]$} & Diseases and Biomarkers & GL-induced acute lung injury (lipopolysaccharides, mouse) \\
\hline 2019 & [9] & Diseases and Biomarkers & Blood platelets in coronary artery disease \\
\hline 2019 & [104] & Diseases and Biomarkers & Premature progesterone rise affecting women's endometrium \\
\hline 2019 & [105] & Diseases and Biomarkers & Plasma-analysis of Metabolic syndrome \\
\hline 2019 & [106] & Diseases and Biomarkers & Alveolar lavage fluid analysis, acute lung injury \\
\hline
\end{tabular}


Table 4. Lipidomic UHPLC-research focusing on cancer

\begin{tabular}{|l|r|l|l|}
\hline Year & Citation & Theme & Subject \\
\hline $\mathbf{2 0 1 7}$ & {$[46]$} & Applications / & Kidney cancer, human patients; Comparison of \\
& & Cancer research & LC/MS;SCF/MS;DIMS \\
\hline $\mathbf{2 0 1 7}$ & {$[107]$} & Applications / & Bee pollen anti-inflammatory properties on cancer cells \\
\hline $\mathbf{2 0 1 7}$ & {$[108]$} & Applications / & Lipid extraction comparison with pancreatic cancer cell line \\
\hline $\mathbf{2 0 1 7}$ & {$[109]$} & Applications / & Cancer cell lines, Quantitative analysis, PC/SM PIS-184 optimization \\
& & Cancer research & \\
\hline $\mathbf{2 0 1 7}$ & {$[110]$} & Cancer research & Urinary exosomes in prostate cancer patients \\
\hline $\mathbf{2 0 1 7}$ & {$[111]$} & Cancer research & Hepatocellular carcinoma in cancer patients \\
\hline $\mathbf{2 0 1 8}$ & {$[112]$} & Cancer research & Review comparing plasmalipid profiles of liver, lung, gastric, \\
& & & Colorectal and thyroid cancer, nanoflow UHPLC \\
\hline $\mathbf{2 0 1 8}$ & {$[113]$} & Cancer research & Non-small cell lung cancer serum biomarker identification \\
\hline
\end{tabular}


Table 5. Extraction and sample purification methods used in lipidomics.

\begin{tabular}{l|l|l}
\hline Class & Technique & Citation \\
\hline Polarity-based & Single solvent extractions & {$[51],[128],[130]$} \\
\hline Polarity-based & Liquid-liquid extractions & {$[51],[128],[130]$} \\
\hline Polarity-based & Single solvent mixtures & {$[51],[128]$} \\
\hline Polarity-based & Bligh and Dyer & {$[129]$} \\
\hline Polarity-based & BUME & {$[120]$} \\
\hline Polarity-based & Folch & {$[119]$} \\
\hline Polarity-based & MTBE & {$[55]$} \\
\hline Polarity-based & QuEChERS & {$[127]$} \\
\hline Polarity-based & Supercritical fluid extraction (SFE) & {$[114],[125]$} \\
\hline Polarity-based & Solid phase extraction (SPE) & {$[58],[125]$} \\
\hline Polarity-based & Superabsorbent polymer extraction (SPE) & {$[127]$} \\
\hline Energy-based & Microwave-assisted extraction (MAE) & {$[114],[125]$} \\
\hline Energy-based & Ultrasonic-assisted extraction (UAE) & {$[114],[125]$} \\
\hline Energy-based & Pressurized liquid extraction & {$[114],[125]$} \\
\hline & & \\
\hline
\end{tabular}


Table 6. Molecular species formed during electrospray ionization of lipids. Ref. [152]

\section{Lipid class}

LPC, PC

LPE, PE

PG

PI

PS

PA

CE

SM

Cholesterol

Cer, GluCer, LacCer

$$
[\mathrm{M}+\mathrm{Na}]^{+}
$$

MG, DG, TG

$$
\left[\mathrm{M}+\mathrm{NH}_{4}\right]^{+},[\mathrm{M}+\mathrm{Na}]^{+}
$$

MGDG, DGDG, SQDG $\left[\mathrm{M}+\mathrm{NH}_{4}\right]^{+},[\mathrm{M}+\mathrm{Na}]^{+}[\mathrm{M}-\mathrm{H}]^{-}$

Fatty acids

$[\mathrm{M}-\mathrm{H}]^{-}$

CL

Cer

$$
\begin{aligned}
& {[\mathrm{M}+\mathrm{H}]^{+},\left[\mathrm{M}+\mathrm{NH}_{4}\right]^{+},[\mathrm{M}-\mathrm{H}]^{-}} \\
& {[\mathrm{M}+\mathrm{Na}]^{+}} \\
& {[\mathrm{M}+\mathrm{H}]^{+},\left[\mathrm{M}+\mathrm{NH}_{4}\right]^{+},[\mathrm{M}-\mathrm{H}]^{-}} \\
& {[\mathrm{M}+\mathrm{Na}]^{+}}
\end{aligned}
$$

$[\mathrm{M}-\mathrm{H}]^{-}$

$\left[\mathrm{M}-\mathrm{H}_{2} \mathrm{O}+\mathrm{H}\right]^{+}$

Negative mode

$[\mathrm{M}+\mathrm{HCOO}]^{-}$ 
Table 7. Classical biological material types. Extension to Jurowski et al.'s Table (Ref. [114]).

\begin{tabular}{|c|c|c|c|}
\hline \multicolumn{2}{|c|}{ Biological samples } & Lipid classes & Reference(s) \\
\hline \multirow{7}{*}{ 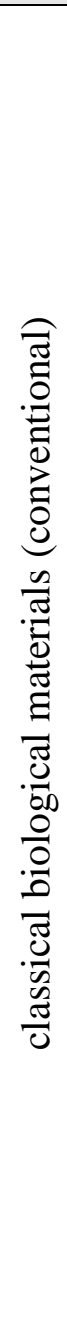 } & Urine & AcCa, MG, DG, TG, PC, PE, LPC, Cer, SM, CEs & {$[5],[110]$} \\
\hline & faecal matter & FA, GPs, STs, SPs, PRs, PKs & {$[50],[156]$} \\
\hline & blood derivatives: & & \\
\hline & Plasma & $\begin{array}{l}\text { FA, MG, DG, TG, PA, PC(+PLs), PE(+PLs), PI, PS, } \\
\text { LPA, LPC, LPE, Cer(+Hex/Hex } 2 / \text { sulfoHex }), \text { SM, S, } \\
\text { SA, cholesterol, CEs, SLs, STs }\end{array}$ & $\begin{array}{l}\text { [5], [46], [52], [57], [58], } \\
{[61],[62],[64],[67],[189],} \\
{[91],[96],[99],[100],[102],} \\
{[103],[105],[155],[157]^{*},} \\
{[158]}\end{array}$ \\
\hline & Serum & $\begin{array}{l}\text { MG, DG, TG, PA, PC(+PLs), PE(+PLs), PG, PI, PS, } \\
\text { LPA, LPC, LPE, Cer(+HexCer/Hex2Cer), SM, S, } \\
\text { CEs, STs, PRs }\end{array}$ & $\begin{array}{l}\text { [5], [15], [62], [76], [90], } \\
{[94],[95],[113]}\end{array}$ \\
\hline & Erythrocytes & PC(+PLs), PE(+PLs), LPC, LPE, Hex2Cer, SM & [46] \\
\hline & blood platelets & $\begin{array}{l}\text { AcCa, FA, DG, TG, PA, PC, PE, PG, PI, PS, LPA, } \\
\text { LPC, LPE, Cer, SM }\end{array}$ & {$[9],[48]^{* *}$} \\
\hline
\end{tabular}

*NIST SRM 1950 plasma, ** Salmonella enterica serovar Typhimurium-infected murine bonemarrow-derived macrophages and thrombin activated blood platelets (human)

Hex: for hexosyl, Hex2: dihexosyl, S: sphingosine, SA: sphinganine 
Table 8. Alternative bioloical materials. Extension to Supplementary Table 1 by Jurovski et al. [114] Hex is an abbreviation for hexosyl, Hex2 for dihexosyl

\begin{tabular}{|c|c|c|c|}
\hline \multicolumn{2}{|c|}{ Biological samples } & \multirow[t]{2}{*}{ Lipid classes } & \multirow{2}{*}{$\begin{array}{l}\text { Reference(s) } \\
{[5],[127]}\end{array}$} \\
\hline \multirow{19}{*}{ 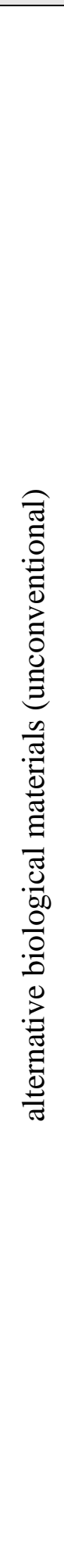 } & Aqueous humor & & \\
\hline & Bile & & [93] \\
\hline & Bronchoalveolar lavage fluid & FA, GLs, GPs, OxGPs, LPLs, SLs & {$[106]$} \\
\hline & Cerebrospinal fluid (CSF) & & [5] \\
\hline & Colon derived suspension & & [8] \\
\hline & Milk & $\mathrm{DG}, \mathrm{TG}, \mathrm{PC}, \mathrm{SM}$ & [47] \\
\hline & Tear drops & & [5] \\
\hline & Cells and cell lines: & & \\
\hline & $\mathrm{x}$ exosomes & FA, GLs, OxGPs, LPLs, SPs, HexCer, CEs, STs & [5], [73], [98], [110] \\
\hline & $\mathrm{x}$ liposomes & FA & [159] \\
\hline & $\mathrm{x}$ alveolar cells & PC, PE(+PLs), PS, LPLs, SM, Cer, HexCer & [160] \\
\hline & $\mathrm{x}$ lipid droplets & & [60] \\
\hline & $\mathrm{x}$ ceratinocytes & FA, GLs, GPs, OxGPs, LPLs, SPs, HexCer, CEs & [74] \\
\hline & Tissues: & & \\
\hline & $\mathrm{x}$ adipose tissue & Cer, SM & [15] \\
\hline & $\mathrm{x}$ lung tissue & $\begin{array}{l}\text { AcCa, FA, TG, PC(+PLS), PE(+PLs), PG, PI, PS, } \\
\text { LPC, LPE, LPI, LPS, Cer, SLs }\end{array}$ & [99], [103] \\
\hline & $\mathrm{X}$ renal tissue & FA, GLs, GPs, SLs, STs, PRs & [95] \\
\hline & $\mathrm{x}$ myocardial tissue & $\begin{array}{l}\text { FA, CL, DG, TG, PA, PC, PE, PG, PI, PS LPC, } \\
\text { LPE, Cer, cholesterol }\end{array}$ & [89], [90] \\
\hline & $\mathrm{x}$ brain tissue & Cer, SM, thromboxane, prostaglandins & {$[15],[53],[155]$} \\
\hline
\end{tabular}




\begin{tabular}{|l|l|l|}
\hline$x$ endometrium & GLs, GPs, LPLs, SPs & {$[104]$} \\
\hline$x$ liver tissue & DG, TG, PA, PC(+PLs), PE(+PLs), PG, PI, PS, & {$[15],[63],[76],[111]$,} \\
& LPA, LPC, LPE, LPG, LPI, LPS, Cer(+HexCer), & {$[127],[155],[160]$} \\
SM, STs & \\
\hline$x$ colonic tissue & & {$[8]$} \\
\hline$x$ tumor tissue & PC(+PLs), PE(+PLs), LPC, LPE, Hex2Cer, SM & {$[46]$} \\
\hline
\end{tabular}


Table 9. Cell culture matrices analyzed with UHPLC-MS techniques.

Hex is an abbreviation for hexosyl, Hex 2 for dihexosyl

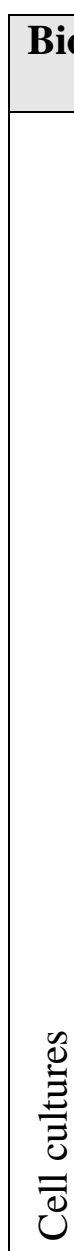

\begin{tabular}{|l}
\hline Bi \\
\hline
\end{tabular}

\begin{tabular}{|c|c|c|}
\hline cal samples & Lipid classes & Reference(s) \\
\hline $\mathrm{HC} 1 *$ & DG, CL, BMP, PE(+OxPE), PG, PS, LPE, PRs & [69] \\
\hline HCC827 (+HCC827-GR) & & [109] \\
\hline HEK 293 & FA, GLs, GPs, PLs, OxGPs, HexCer, CEs, STs & [73] \\
\hline HeLa & $\begin{array}{l}\text { CL, DG, TG, PC, PE, PG, PI, PS, LPC, LPE, } \\
\text { Cer(+HexCer), SM }\end{array}$ & [70], [130] \\
\hline Huh7 & LPC, LPE & {$[92]$} \\
\hline HTC-116 & & {$[109]$} \\
\hline Huh7 & LPC, LPE & [92] \\
\hline MDCK (+MDCK-GR) & & [15], [109] \\
\hline OVCAR-3 & & [109] \\
\hline OxWR* & CL, DG, BMP, PE, PG, PS, LPE, PRs & [69] \\
\hline PANC-1 & CE, DG, TG, PC, PE, PI, PS, LPC, Cer, SM & {$[108]$} \\
\hline PC-9 (+PC-9-GR) & & {$[109]$} \\
\hline $\mathrm{PC} 12$ & PC, PE, PI, PS & {$[72]$} \\
\hline RAW 264.7 (macrophages)** & GLs, PA, PC, PE, PG, PI, PS, LPLs, SPs, CEs, cholesterol & [66], [107] \\
\hline SKOV-3 & & {$[109]$} \\
\hline Bladder cancer cell & & {$[155]$} \\
\hline Melanoma B16*** & Cer, SM & {$[15]$} \\
\hline $\begin{array}{l}\text { Primary CD4+T } \\
\text { lymphocytes*** }\end{array}$ & Cer, SM & {$[15]$} \\
\hline Yeast & PA, PC, PE, PG, PI, PS & {$[140]$} \\
\hline
\end{tabular}

*anaerobic, oxalobacter formigenes ; **widest lipid range ; ***mouse 
Table 10. Common lipids according to research topic and identification approach. When "Identified lipids" are indicated as a sum without any explanation, it means that the first number is the amount of lipids found in positive and the second in negative mode (positive+negative).

\begin{tabular}{|c|c|c|c|c|c|c|c|c|c|c|}
\hline Citation & Theme & $\begin{array}{l}\text { T/relT/U } \\
\text { T }\end{array}$ & & $\frac{1}{b}$ & & & & & $\begin{array}{l}\text { Identified } \\
\text { lipids }\end{array}$ & Bio-markers \\
\hline [47] & Method development & UT & 2 & 1 & & & 1 & & 429 & \\
\hline [161] & Method development & relT & 1 & 4 & & & 1 & & & \\
\hline [160] & Method development & UT & 2 & 3 & $*$ & 2 & 1 & 1 & & \\
\hline [160] & Method development & UT & 2 & 4 & $*$ & 2 & 2 & & & \\
\hline [160] & Method development & UT & & 3 & $*$ & 2 & 2 & 1 & & \\
\hline [140] & Method development & UT & & 6 & $*$ & & & & 436 & \\
\hline [127] & Method development & T/UT & 2 & 6 & & 5 & 2 & & 207 & \\
\hline [130] & Method development & UT & 2 & 4 & & 2 & * & & $292+206$ & \\
\hline [157] & Method development & $\mathrm{T}$ & 1 & 4 & & 2 & 1 & & 22 & \\
\hline [155] & Method development & PT & 2 & 2 & $*$ & 2 & 2 & 2 & $515+630+6$ & $0[\mathrm{D}]$ \\
\hline [189] & Method development & UT & 2 & 3 & & 1 & 2 & & 104 & \\
\hline [159] & Method development & UT & & & & & & & 403 & \\
\hline [48] & Method development & UT & & & & & & & $85[\mathrm{E}]$ & \\
\hline [108] & $\begin{array}{l}\text { Method development / cancer } \\
\text { research }\end{array}$ & UT & 2 & 4 & & 2 & 2 & & $226-414$ & \\
\hline [63] & $\begin{array}{l}\text { Method development / } \\
\text { Metabolomics }\end{array}$ & UT & $*$ & $*$ & & & & & 83 & 8 \\
\hline [46] & $\begin{array}{l}\text { Method development / cancer } \\
\text { research }\end{array}$ & T/UT & 3 & 5 & & 3 & 2 & 1 & 132 & \\
\hline [110] & Cancer research & UT & 2 & 1 & $*$ & & 2 & 1 & 286 & 34 \\
\hline [111] & Cancer research & UT & 2 & 2 & & & & & & 50 \\
\hline
\end{tabular}




\begin{tabular}{|c|c|c|c|c|c|c|c|c|c|c|}
\hline [113] & Cancer research & T/UT & 3 & 5 & $*$ & & 3 & 2 & 493 & $14+10+2[\mathrm{~B}]$ \\
\hline [92] & Diseases and Biomarkers & UT & & & & 2 & & & 24 & \\
\hline [101] & Diseases and Biomarkers & UT & & 4 & & 2 & 1 & & 129 & \\
\hline [94] & Diseases and Biomarkers & UT & 3 & 6 & & 3 & 3 & & 749 & 16 \\
\hline [89] & Diseases and Biomarkers & UT & 1 & 2 & & & & & 12 & 9 \\
\hline [95] & Diseases and Biomarkers & UT & $*$ & $*$ & & & * & & 179 & \\
\hline [95] & Diseases and Biomarkers & & * & $*$ & & & * & & 196 & \\
\hline [90] & Diseases and Biomarkers & UT & 1 & 2 & & 1 & 1 & & 746 & 11 \\
\hline [90] & Diseases and Biomarkers & & 2 & 2 & & 1 & 1 & & & \\
\hline [91] & Diseases and Biomarkers & UT & 3 & 5 & & & 4 & & $261+39$ & 7 \\
\hline [96] & Diseases and Biomarkers & UT & 1 & 2 & $*$ & 2 & 2 & & 365 & 19 \\
\hline [97] & Diseases and Biomarkers & UT & 2 & 2 & $*$ & 2 & 2 & 3 & 363 & 28 \\
\hline [98] & Diseases and Biomarkers & UT & 1 & 1 & & & & & Features & Features \\
\hline [5] & Diseases and Biomarkers & & 2 & 4 & & & 1 & & & $>25$ \\
\hline [102] & Diseases and Biomarkers & $\mathrm{relT} / \mathrm{T}$ & & & & & & & $81[\mathrm{~A}]$ & $17[\mathrm{~A}]$ \\
\hline [103] & Diseases and Biomarkers & UT & & & & & & & & 77 \\
\hline [9] & Diseases and Biomarkers & UT & 2 & 5 & & 3 & 2 & & & 77 \\
\hline [99] & Diseases and Biomarkers & UT & 1 & 5 & $*$ & 3 & 1 & & $188+62$ & 87 \\
\hline [100] & Diseases and Biomarkers & & 2 & 1 & & 1 & 1 & & & \\
\hline [104] & Diseases and Biomarkers & UT & 1 & 4 & & 1 & 1 & & 1026 & 25 \\
\hline [105] & Diseases and Biomarkers & T/UT & 2 & 2 & $*$ & 1 & 2 & & 55 & 55 \\
\hline [106] & Diseases and Biomarkers & UT & 1 & 3 & $*$ & 2 & & & $38+31$ & $11+14$ \\
\hline [45] & Drug-testing & UT & 1 & 1 & & 1 & 1 & & 155 & \\
\hline [56] & Foodstuff profiling & $\mathrm{T}$ & 1 & 4 & & 2 & 1 & & 81 & \\
\hline [67] & Metabolomics & UT & 1 & 5 & & 1 & * & & 61 & \\
\hline [68] & Metabolomics & UT & & & & 4 & & & & \\
\hline [62] & Metabolomics & UT & 2 & 4 & & $*$ & & & & \\
\hline
\end{tabular}




\begin{tabular}{|c|c|c|c|c|c|c|c|c|c|c|c|}
\hline$[65]$ & Metabolomics & UT & 2 & 5 & & & & 1 & & 178 & \\
\hline [69] & Metabolomics & UT & 1 & 4 & $*$ & 1 & 1 & & & 97 & \\
\hline [69] & Metabolomics & & 1 & 3 & $*$ & & 1 & & & & \\
\hline [158] & Metabolomics & UT & 1 & 2 & & & 2 & 1 & & 226 & \\
\hline [70] & Metabolomics & UT & & 5 & & & & 1 & & $249+451$ & \\
\hline [71] & Metabolomics & UT & 1 & 1 & & & & & & 7 & \\
\hline [72] & Metabolomics & $\mathrm{T}$ & & 4 & & & & & & 22 & 5 \\
\hline$[66]$ & Metabolomics & UT & 3 & 6 & & & 6 & 7 & & 523 & \\
\hline [73] & Metabolomics & UT & 1 & 7 & $*$ & $*$ & 5 & 2 & 1 & 642 & 53 \\
\hline$[74]$ & Metabolomics & UT & 2 & 5 & $*$ & & 4 & 2 & 1 & 611 & 440 \\
\hline$[75]$ & Metabolomics & UT & & 6 & $*$ & & 3 & 2 & & N/A & $\geq 43$ \\
\hline [76] & Metabolomics & $\mathrm{T}$ & 1 & 4 & $*$ & & 1 & 2 & & & $\begin{array}{c}126+124 \\
{[\mathrm{E}]}\end{array}$ \\
\hline [87] & Metabolomics & UT & & 7 & $*$ & & 1 & 2 & & 119 & 38 \\
\hline [50] & Physiology & T/UT & & $*$ & & & & $*$ & & Features & \\
\hline [58] & Physiology & $\mathrm{T}$ & & & & & & & & & \\
\hline [15] & Physiology & $\mathrm{T}$ & & & & & & 2 & & 45 & \\
\hline [59] & Physiology & UT & 1 & 6 & $*$ & & 2 & 2 & & 283 & \\
\hline [52] & Physiology & UT & & & & & & & & 12 & \\
\hline [53] & Physiology & $\mathrm{T}$ & & & & & & & & 5 & \\
\hline [107] & Physiology & UT & & & * & & & 1 & & $184+150$ & \\
\hline [57] & Physiology & UT & 2 & 1 & & & & & & & 35 \\
\hline [61] & Physiology & UT & 3 & 5 & & & 2 & 1 & & $529+179$ & \\
\hline
\end{tabular}

*: Lipid subgroups could not be determined

T/relT/UT: targeted/relatively targeted/untargeted

[A] for hyperlipidemia: plasma(74, 57 biomarkers), $\operatorname{VLDL}(74,52$ biomarkers), LDL(76, 42 biomarkers), HDL(73, 41 biomarkers) 
[B] non-small cell lung cancer+lung benign disease+healthy controls

[C] metabolites

[D] in plasma+tissue+cell

[E]: serum+liver 
Table 11. Extension of lipid classes analyzed in different research articles (2017-2019)

\begin{tabular}{|c|c|c|c|c|c|c|c|c|c|c|c|c|c|}
\hline Citation & Theme & $\begin{array}{l}\text { T/relT } \\
\text { /UT/PT }\end{array}$ & $\sum_{\frac{1}{4}}^{n}$ & 㟧 & 气 & $\tilde{\omega}$ & $\frac{u}{a}$ & $\underline{\underline{n}}$ & 몸 & $\stackrel{\text { ă }}{a}$ & $\stackrel{y}{\partial}$ & $\begin{array}{l}\text { Identified } \\
\text { lipids }\end{array}$ & $\begin{array}{l}\text { Bio- } \\
\text { markers }\end{array}$ \\
\hline [47] & Method development & UT & & & & & & & & & & 429 & \\
\hline [161] & Method development & relT & & & & & & & & & & & \\
\hline [160] & Method development & UT & & & & & & & & & & & \\
\hline [160] & Method development & UT & & & & & & & & & & & \\
\hline [160] & Method development & UT & & & & & & & & & & & \\
\hline [140] & Method development & UT & & & & & & & & & & 436 & \\
\hline [127] & Method development & T/UT & & & $*$ & $*$ & & & & & & 207 & \\
\hline [130] & Method development & UT & & $*$ & & & & & & & & $292+206$ & \\
\hline [157] & Method development & $\mathrm{T}$ & 1 & & & & & & & & & 22 & \\
\hline [155] & Method development & PT & & $*$ & & & & & & & & \multicolumn{2}{|c|}{$515+630+640[\mathrm{D}]$} \\
\hline [189] & Method development & UT & & $*$ & & & & & & & & 104 & \\
\hline [159] & Method development & UT & $*$ & & & & & & & & & 403 & \\
\hline [48] & Method development & UT & $*$ & & & & & & & & & 85 & \\
\hline [108] & $\begin{array}{l}\text { Method development } \\
\text { / cancer research }\end{array}$ & UT & & $*$ & & & & & & & & $226-414$ & \\
\hline [63] & $\begin{array}{l}\text { Method development } \\
\text { / Metabolics }\end{array}$ & UT & & & $*$ & & & & & & & 83 & 8 \\
\hline$[46]$ & $\begin{array}{l}\text { Method development } \\
\text { /cancer research }\end{array}$ & T/UT & & $*$ & & & & & & & & 132 & \\
\hline [110] & Cancer research & UT & & $*$ & & & & & & & & 286 & 34 \\
\hline [111] & Cancer research & UT & & & & & & & & & & & 50 \\
\hline [113] & Cancer research & T/UT & & $*$ & & & & & & & & 493 & $\begin{array}{c}14+10+2 \\
{[B]}\end{array}$ \\
\hline [92] & Diseases & UT & & & & & & & & & & 24 & \\
\hline [101] & Diseases & UT & & & & & & & & & & 129 & \\
\hline [94] & Diseases & UT & & $*$ & $*$ & & & & & & & 749 & 16 \\
\hline [89] & Diseases & UT & & & & & & & 1 & & & 12 & 9 \\
\hline [95] & Diseases & UT & $*$ & & $*$ & $*$ & $*$ & & & & & 179 & \\
\hline [95] & Diseases & & $*$ & & $*$ & $*$ & $*$ & & & & & 196 & \\
\hline [90] & Diseases & UT & & & & & & & & & & 746 & 11 \\
\hline [90] & Diseases & & & & & & & & & & & & \\
\hline [91] & Diseases & UT & & & & $*$ & & & & & & $261+39$ & 7 \\
\hline [96] & Diseases & UT & & & & $*$ & & & & & & 365 & 19 \\
\hline [97] & Diseases & UT & & & & & & & & & & 363 & 28 \\
\hline [98] & Diseases & UT & 1 & & & & & & & & & Features & Features \\
\hline [5] & Diseases & & 1 & $*$ & & & & & & & & & $>25$ \\
\hline [102] & Diseases & $\mathrm{relT} / \mathrm{T}$ & 1 & & & & & & $*$ & $*$ & $*$ & $81[\mathrm{~A}]$ & $17[\mathrm{~A}]$ \\
\hline [103] & Diseases & UT & & & $*$ & & & & & & & & 77 \\
\hline [9] & Diseases & UT & 2 & & & & & & & & & & 77 \\
\hline [99] & Diseases & UT & 2 & & $*$ & & & & & & & $188+62$ & 87 \\
\hline [100] & Diseases & UT & 1 & & & & & & & & & $188[C]$ & \\
\hline [100] & Diseases & & 1 & & & & & & & & & & \\
\hline [45] & Drug-testing & UT & & & & & & & & & & 155 & \\
\hline [104] & Diseases & UT & & & & & & & & & & 1026 & 25 \\
\hline
\end{tabular}




\begin{tabular}{|c|c|c|c|c|c|c|c|c|c|c|c|c|}
\hline [105] & Diseases & T/UT & & $*$ & $*$ & & & & & & 55 & 55 \\
\hline [106] & Diseases & UT & $*$ & & $*$ & & & & & & $38+31$ & $11+14$ \\
\hline [56] & Foodstuff profiling & $\mathrm{T}$ & & & & & & & & & 81 & \\
\hline [67] & Metabolomics & UT & $*$ & & & & & & & & 61 & \\
\hline [68] & Metabolomics & UT & & & & & & & & & & \\
\hline [62] & Metabolomics & UT & & $*$ & & & & & & & & \\
\hline [65] & Metabolomics & UT & & & 3 & & & & & & 178 & \\
\hline [69] & Metabolomics & UT & 1 & & & & 1 & & & & 97 & \\
\hline [69] & Metabolomics & & 1 & & & & 1 & & & & & \\
\hline [158] & Metabolomics & UT & & & & & & & $*$ & $*$ & 226 & \\
\hline [70] & Metabolomics & UT & 1 & & & & & & & & $249+451$ & \\
\hline [71] & Metabolomics & UT & & & & 1 & & & & & 7 & \\
\hline [72] & Metabolomics & $\mathrm{T}$ & & & & & & & & & 22 & 5 \\
\hline [66] & Metabolomics & UT & & $*$ & & & & & & & 523 & \\
\hline [73] & Metabolomics & UT & $*$ & $*$ & & $*$ & & & & $*$ & 642 & 53 \\
\hline [74] & Metabolomics & UT & 2 & $*$ & & & & & & & 611 & 440 \\
\hline [75] & Metabolomics & UT & $*$ & & & & & & & & $\mathrm{~N} / \mathrm{A}$ & $\geq 43$ \\
\hline [76] & Metabolomics & $\mathrm{T}$ & & & & & & & & & $126+124$ & \\
\hline [87] & Metabolomics & UT & & & & & & & & & 119 & 38 \\
\hline [50] & Physiology & T/UT & $*$ & & & $*$ & $*$ & $*$ & & & Features & \\
\hline [58] & Physiology & $T$ & $*$ & & & & & & & & & \\
\hline [15] & Physiology & $T$ & & & & & & & & & 45 & \\
\hline [59] & Physiology & UT & & & 1 & & & & & & 283 & \\
\hline [52] & Physiology & UT & & & & $* *$ & & & & & 12 & \\
\hline [53] & Physiology & $T$ & $* * *$ & & & & & & & & 5 & \\
\hline [107] & Physiology & UT & 2 & & & & & & & & $184+150$ & \\
\hline [57] & Physiology & UT & & & & & & & & & & 35 \\
\hline [61] & Physiology & UT & $*$ & & & & & & & & $529+179$ & \\
\hline
\end{tabular}

* Lipid classes not counted

**15 steroids

\section{$* * * 5$ prostaglandins}

[A] for hyperlipidemia: plasma(74, 57 biomarkers), $\operatorname{VLDL}(74,52$ biomarkers),

LDL(76, 42 biomarkers), HDL(73, 41 biomarkers)

[B] non-small cell lung cancer+lung benign disease+healthy controls

[C] metabolites

[D] in plasma+tissue+cell

[E]: serum+liver 
Click here to download high resolution image

a

$$
\begin{aligned}
& \text { Cellular fatty } \\
& \text { acidsynthesis } \\
& \text { machinery }
\end{aligned}
$$

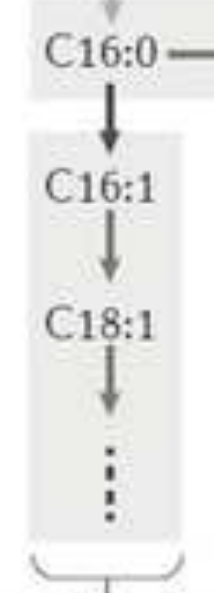

n-7 family

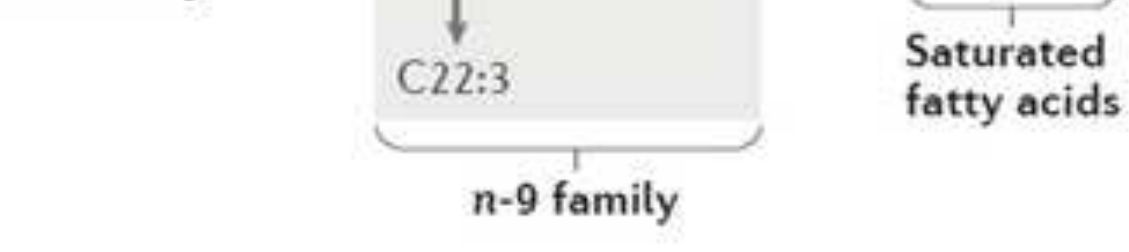

b

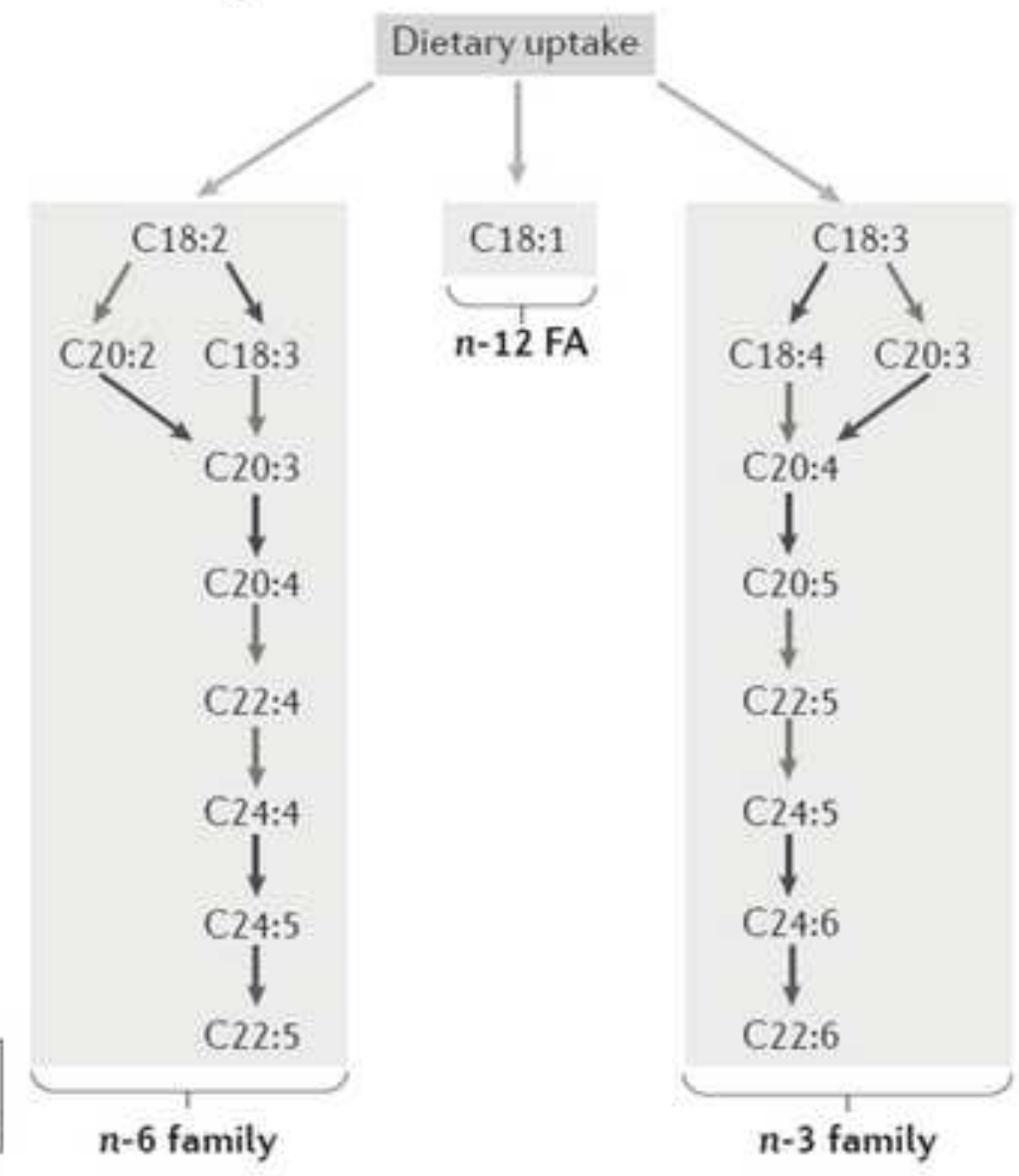




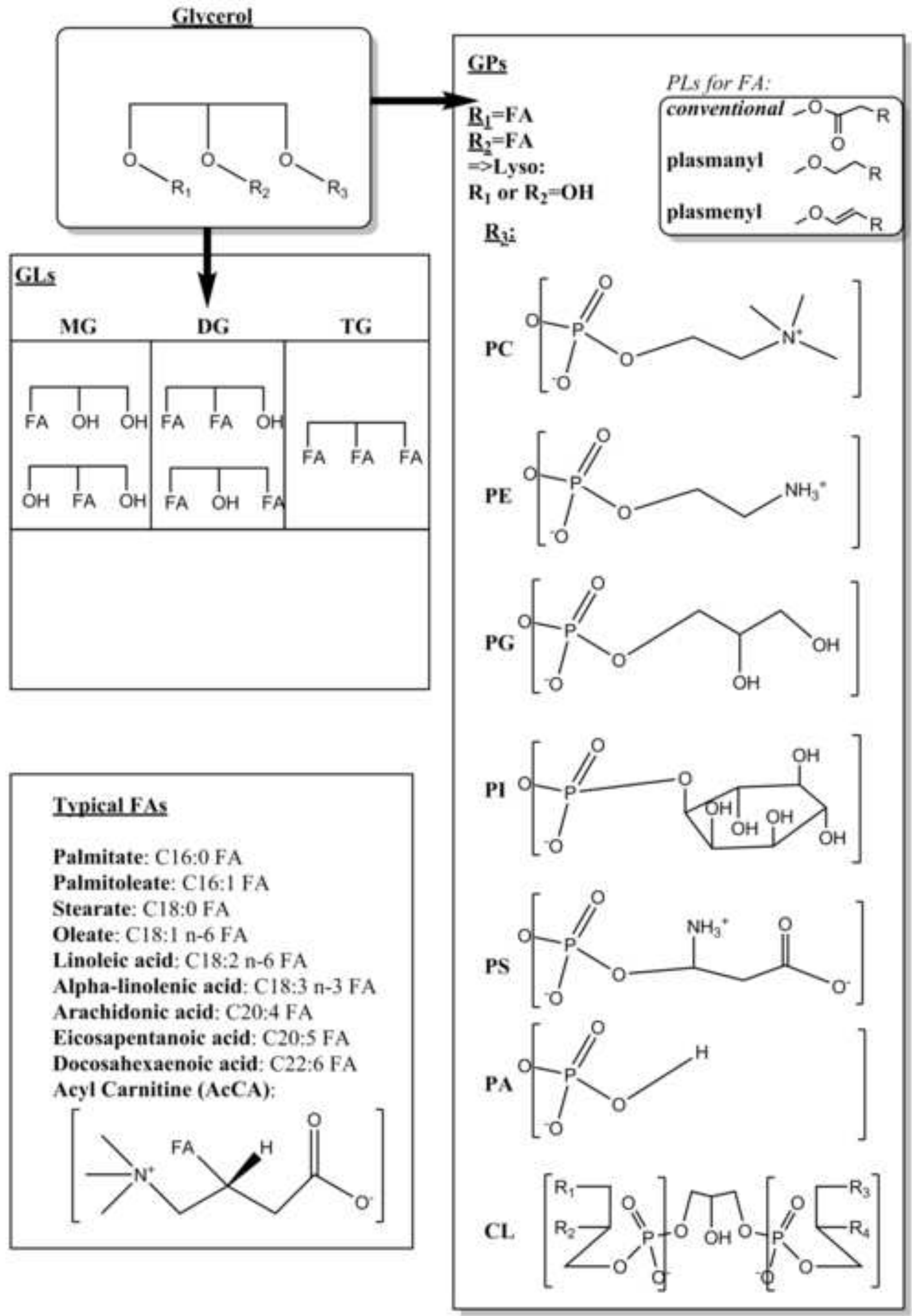




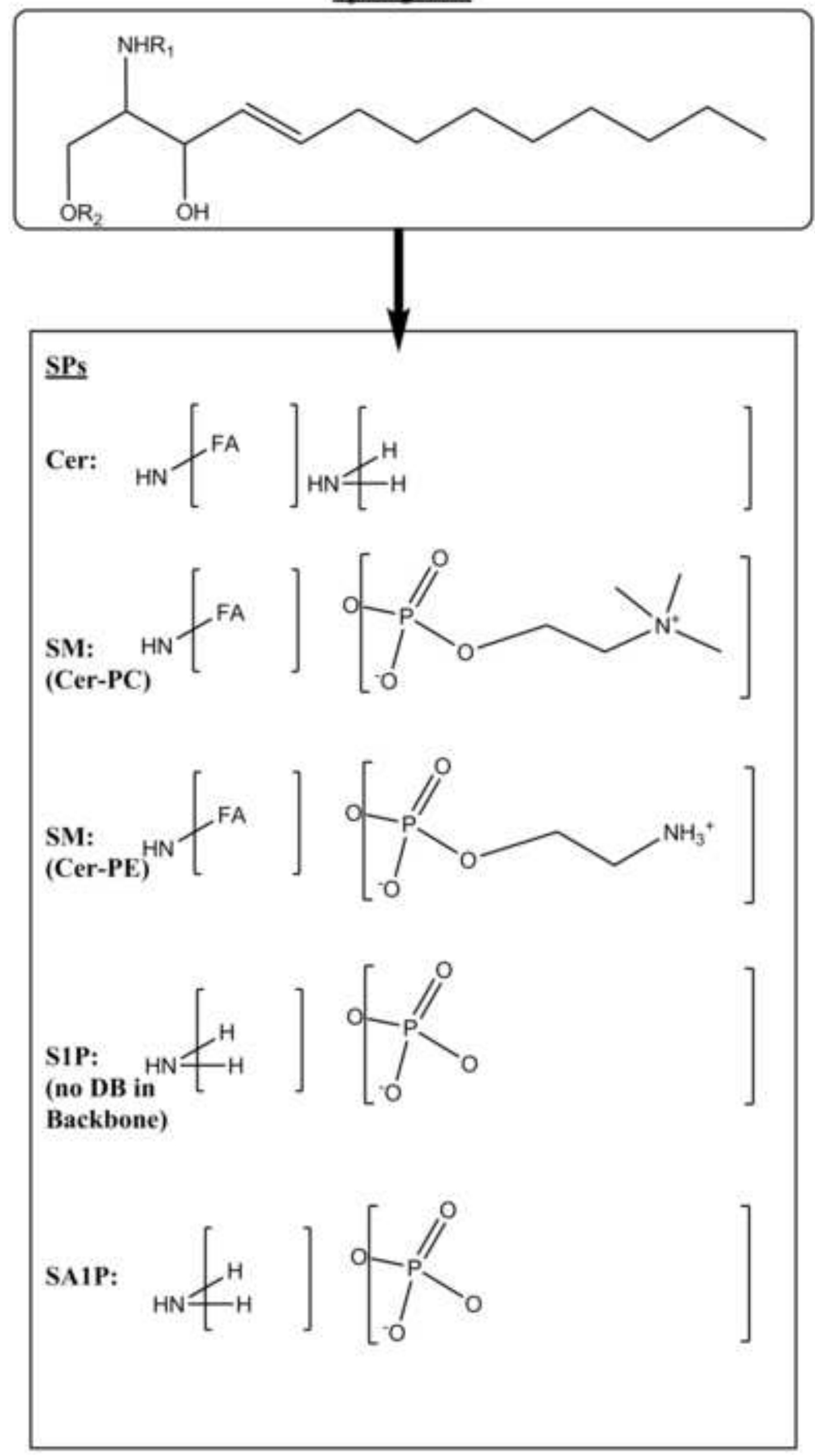



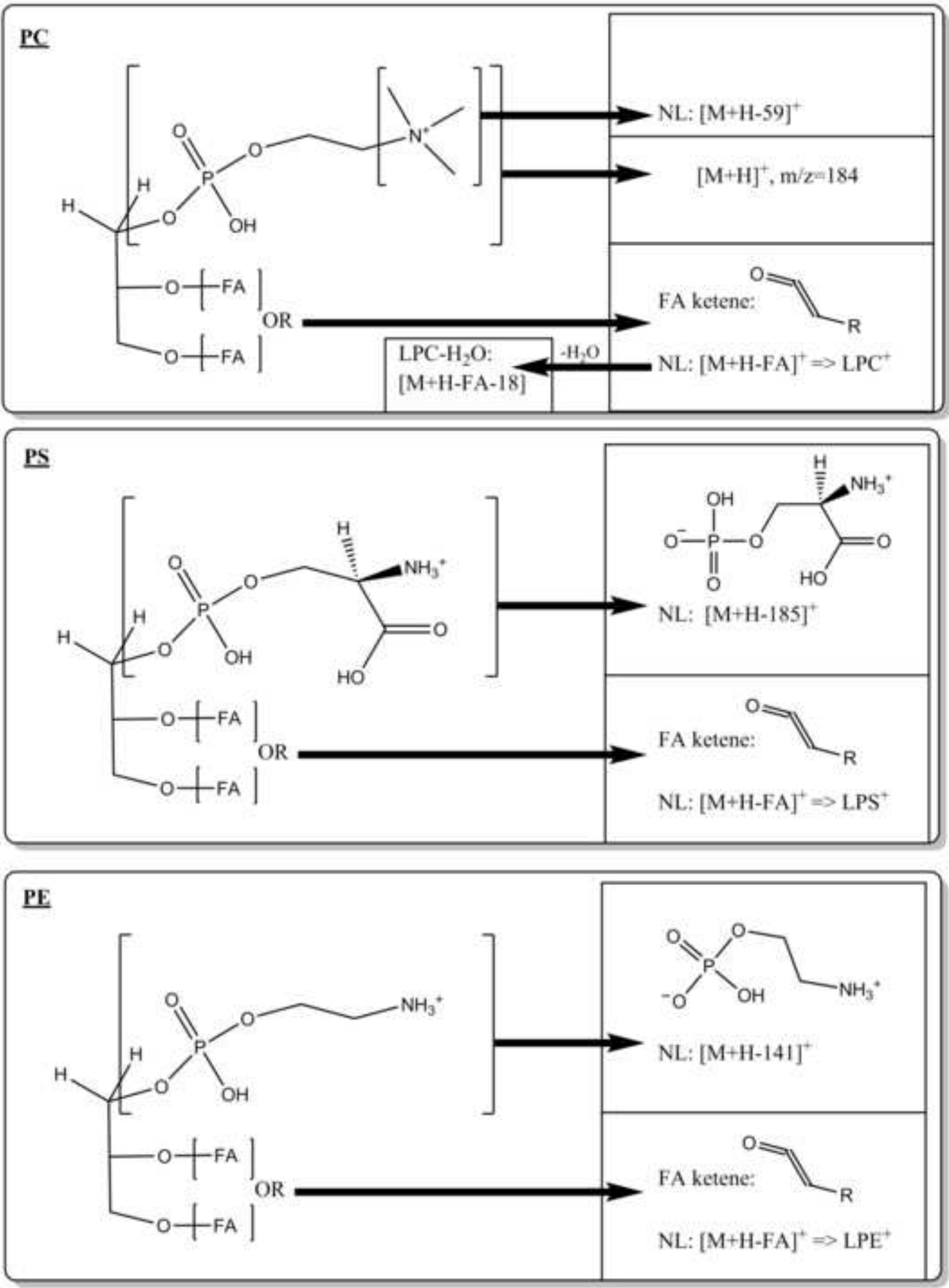

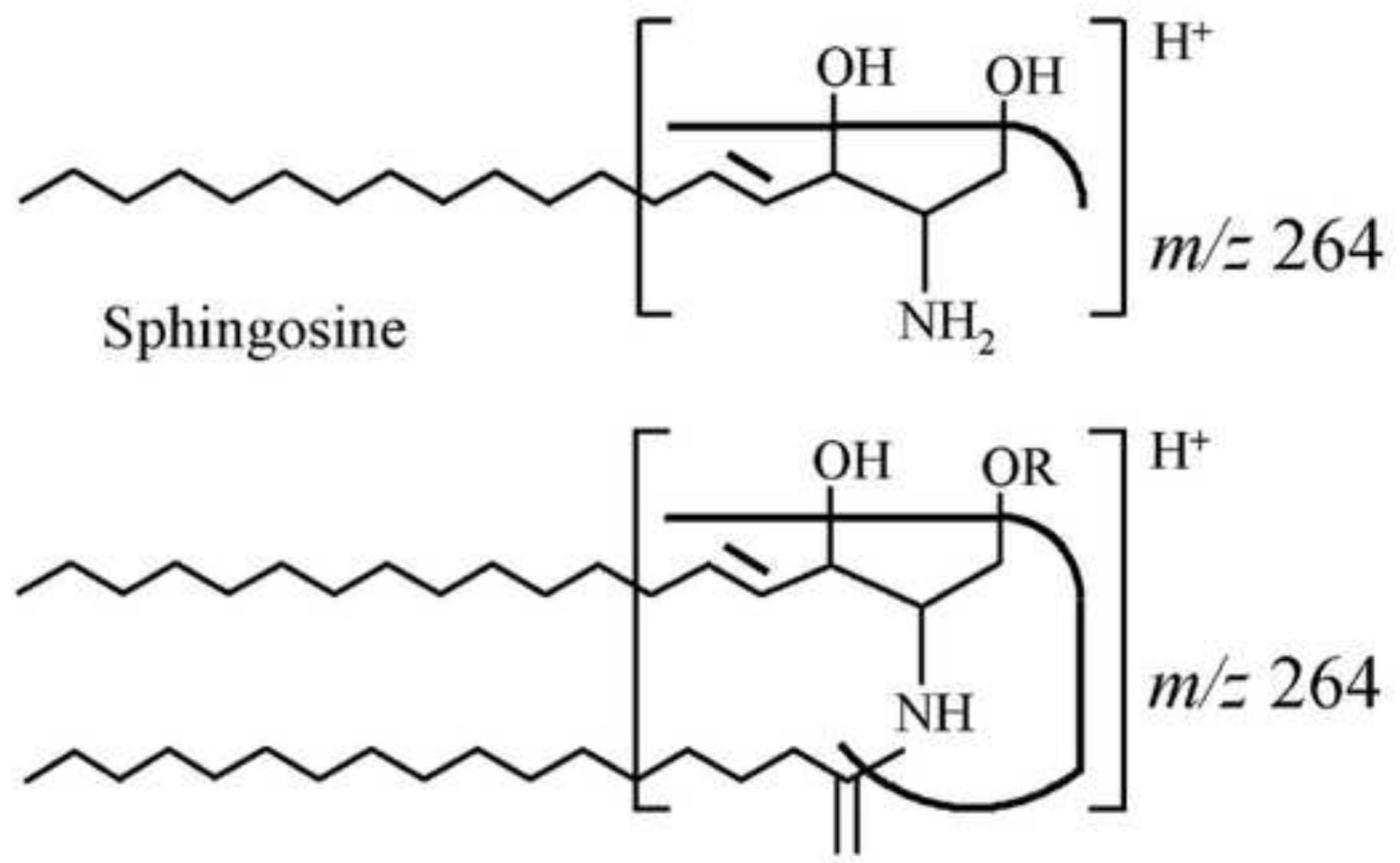

Ceramide $(\mathrm{R}=\mathrm{H})$ \&

O

Glycosphingolipids $(\mathrm{R}=$ carbohydrate $)$

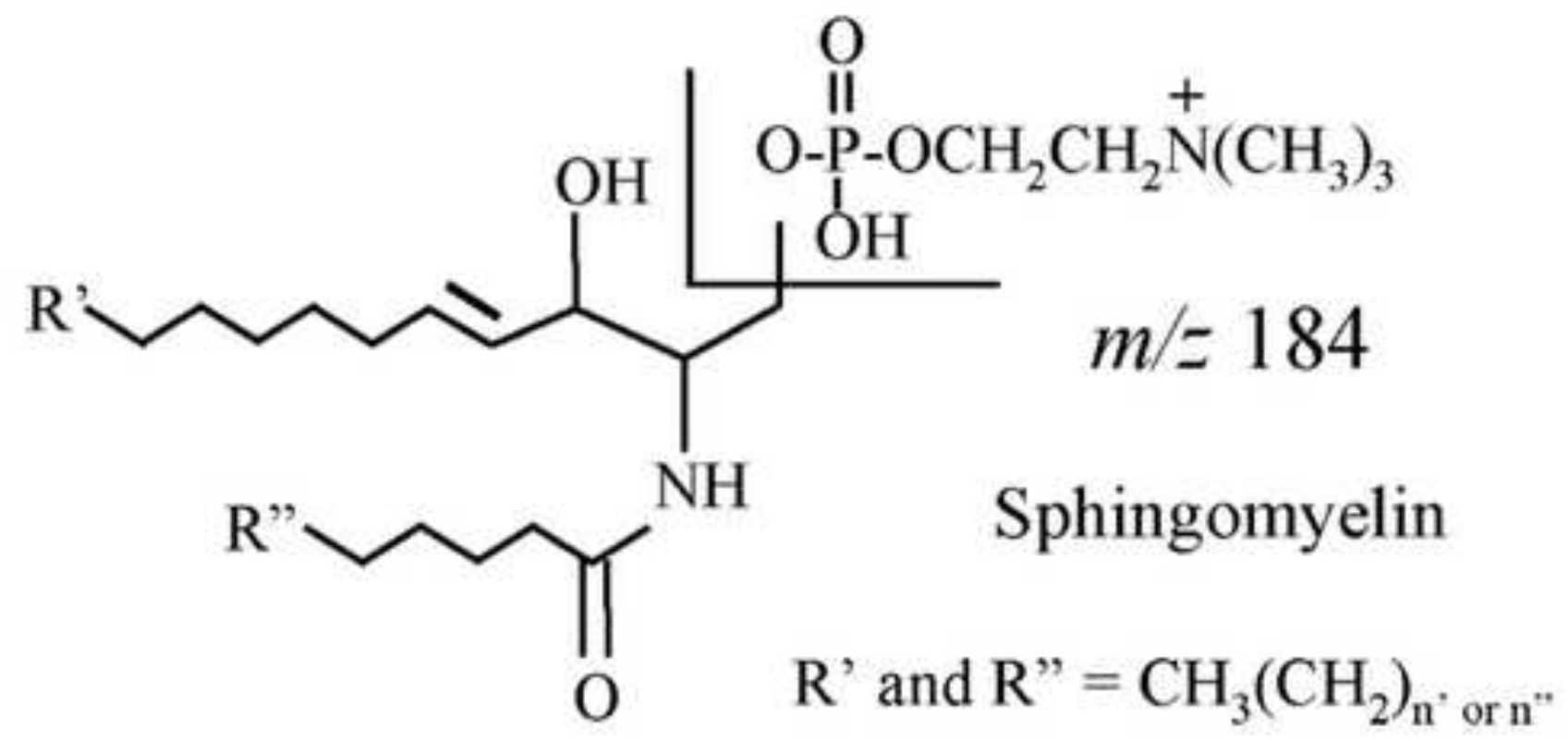




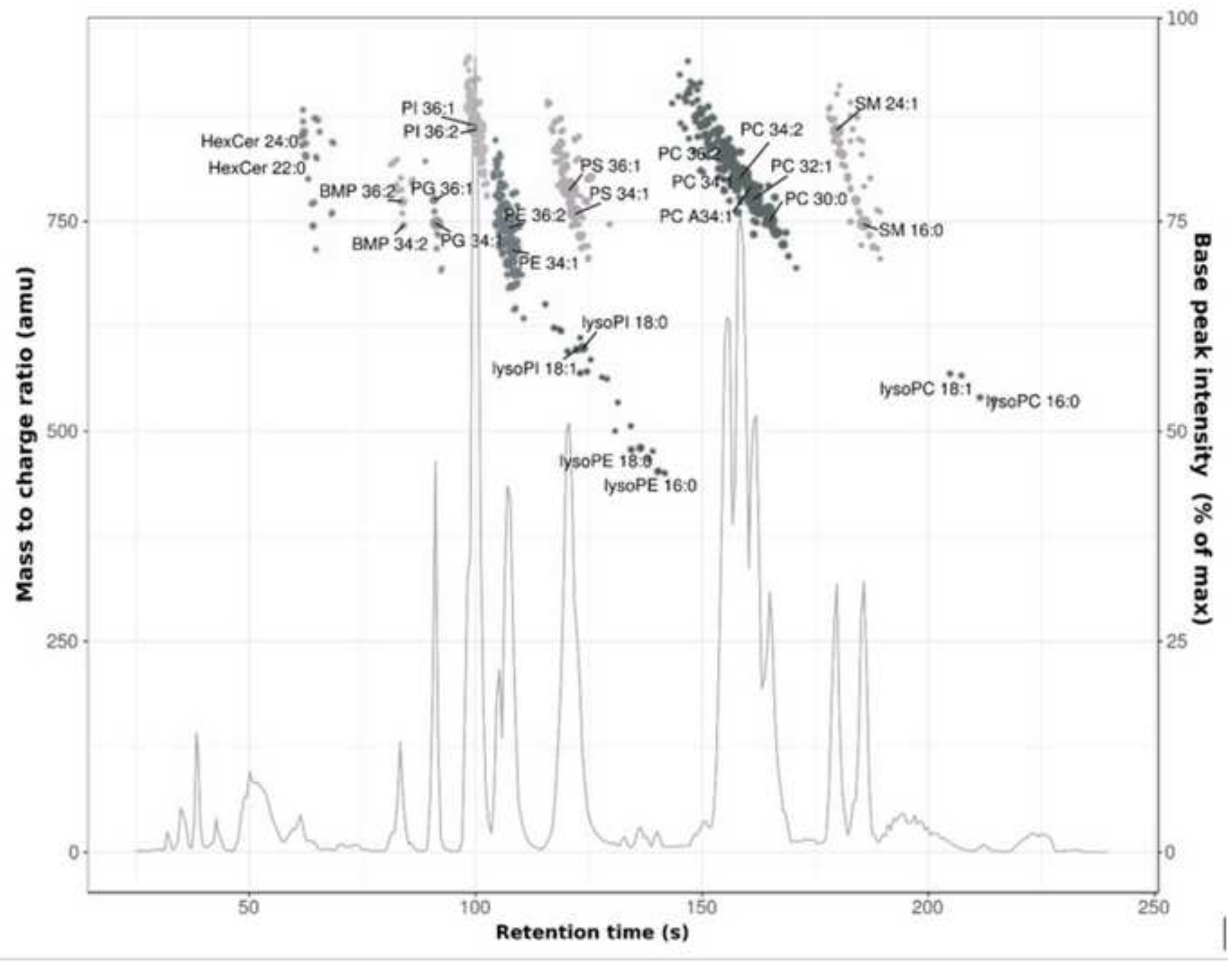


(a)

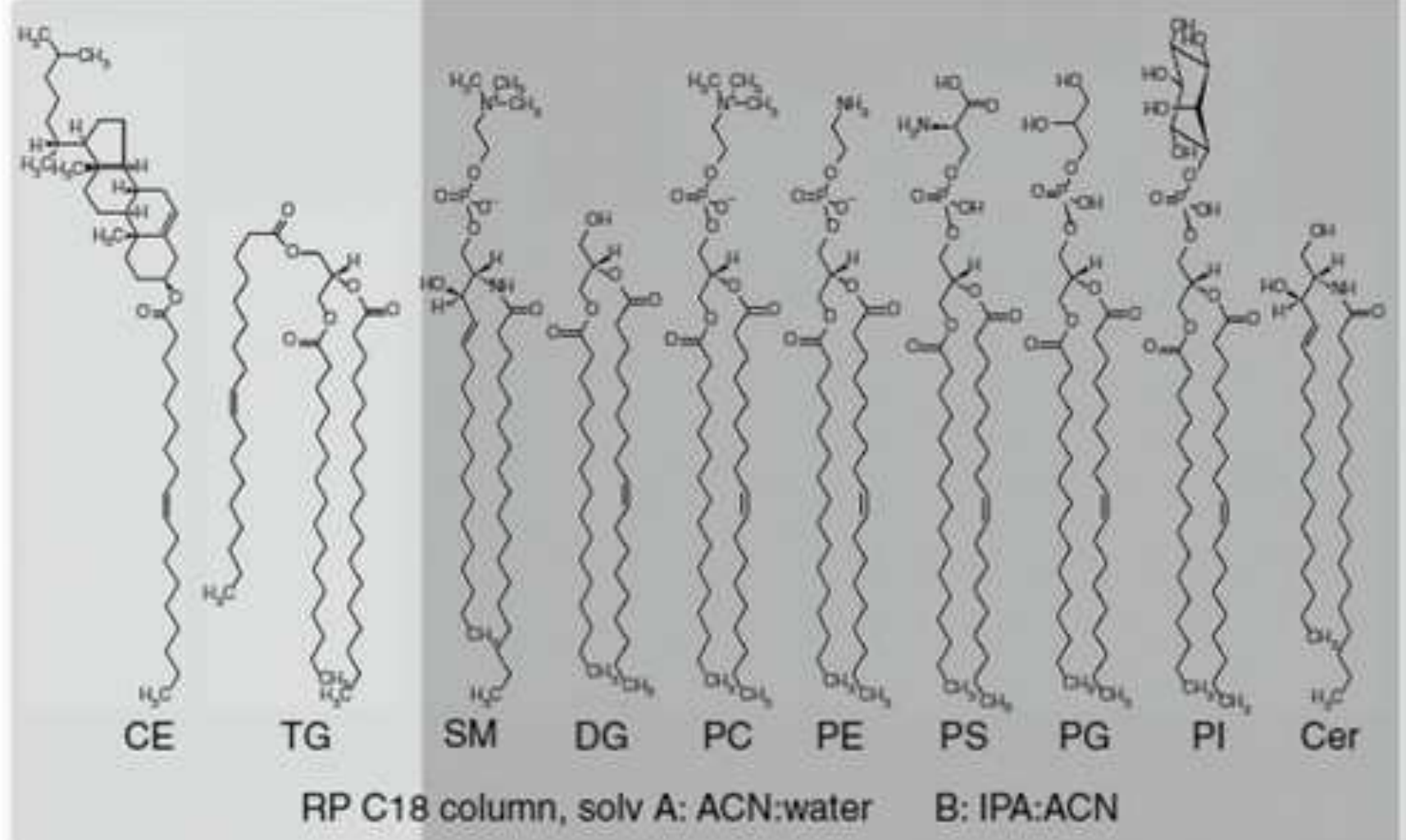

NP Silica, solv A\&B IPA:Hexane

(b)

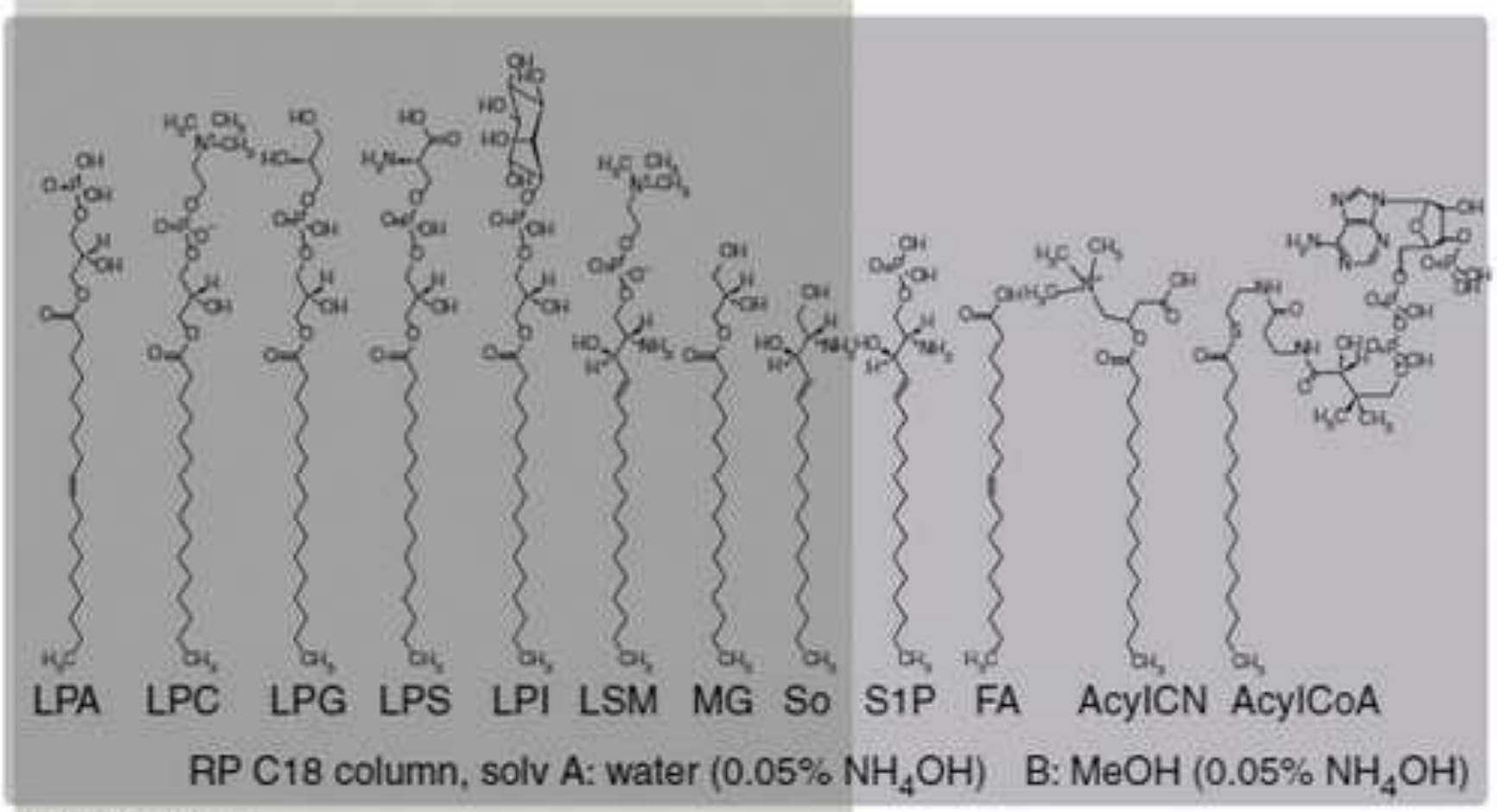

HILIC column,

A: $0.2 \%$ formic acid, $200 \mathrm{mM} \mathrm{NH}_{4} \mathrm{HCOO}$ in water

B: $0.2 \%$ formic acid in $\mathrm{ACN}$ 


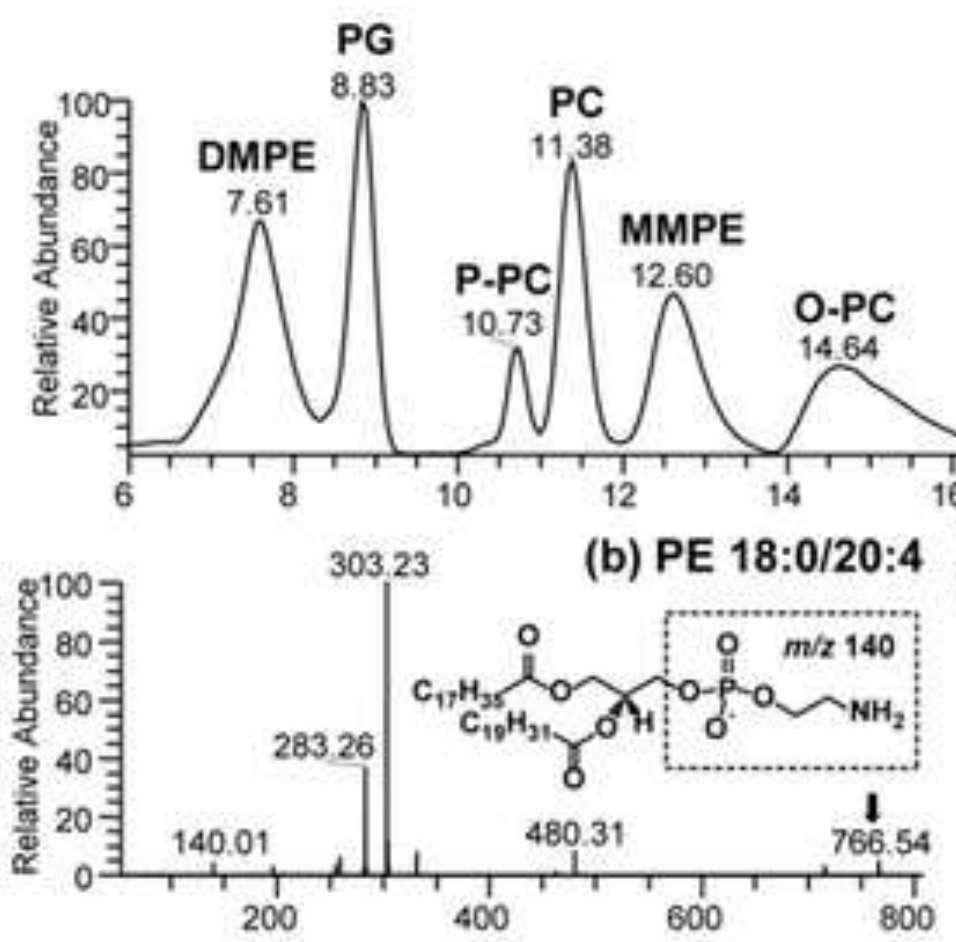

(a) negHILIC-MS chromatogram
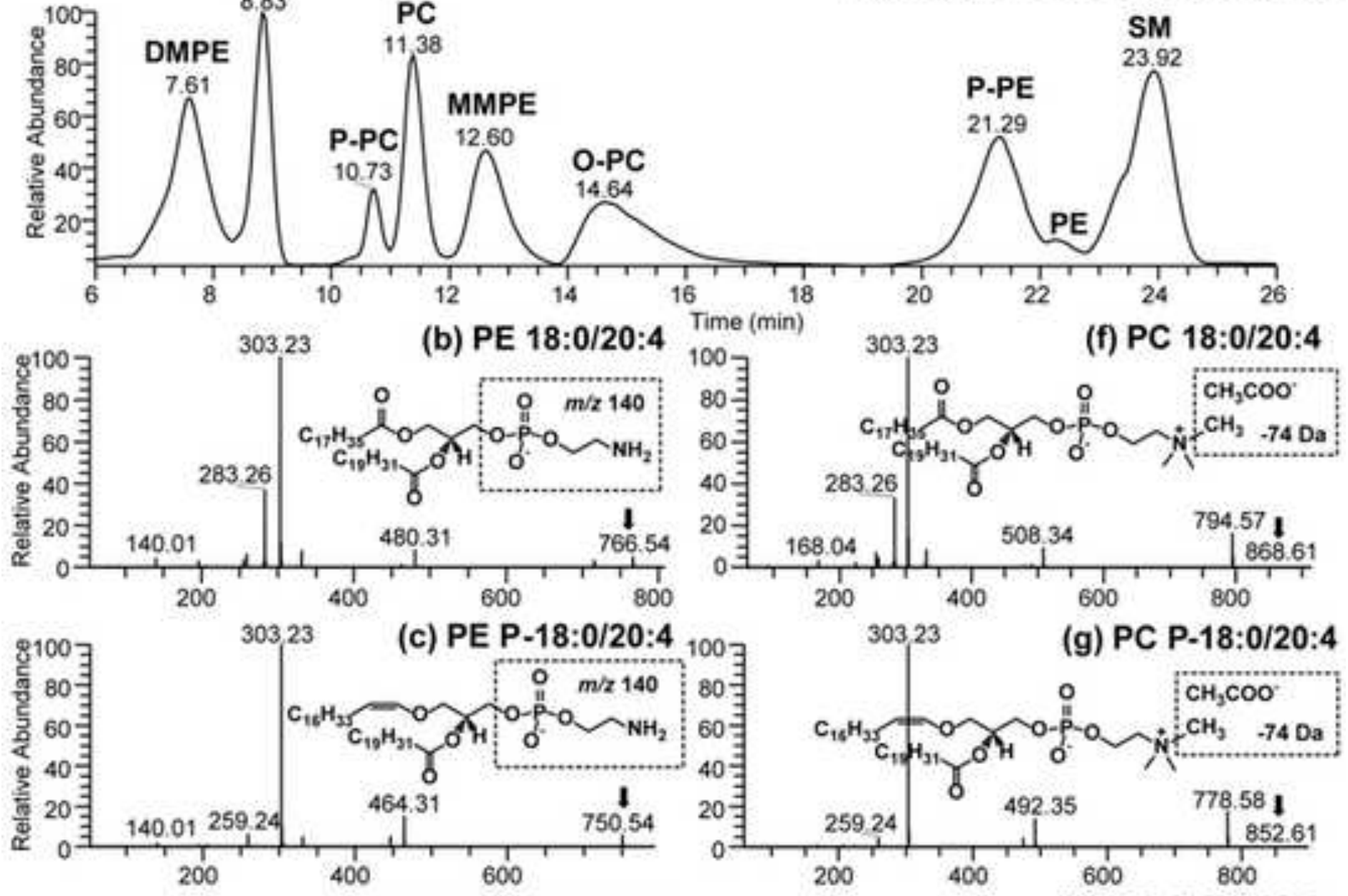

(d) MMPE 16:0/16:0
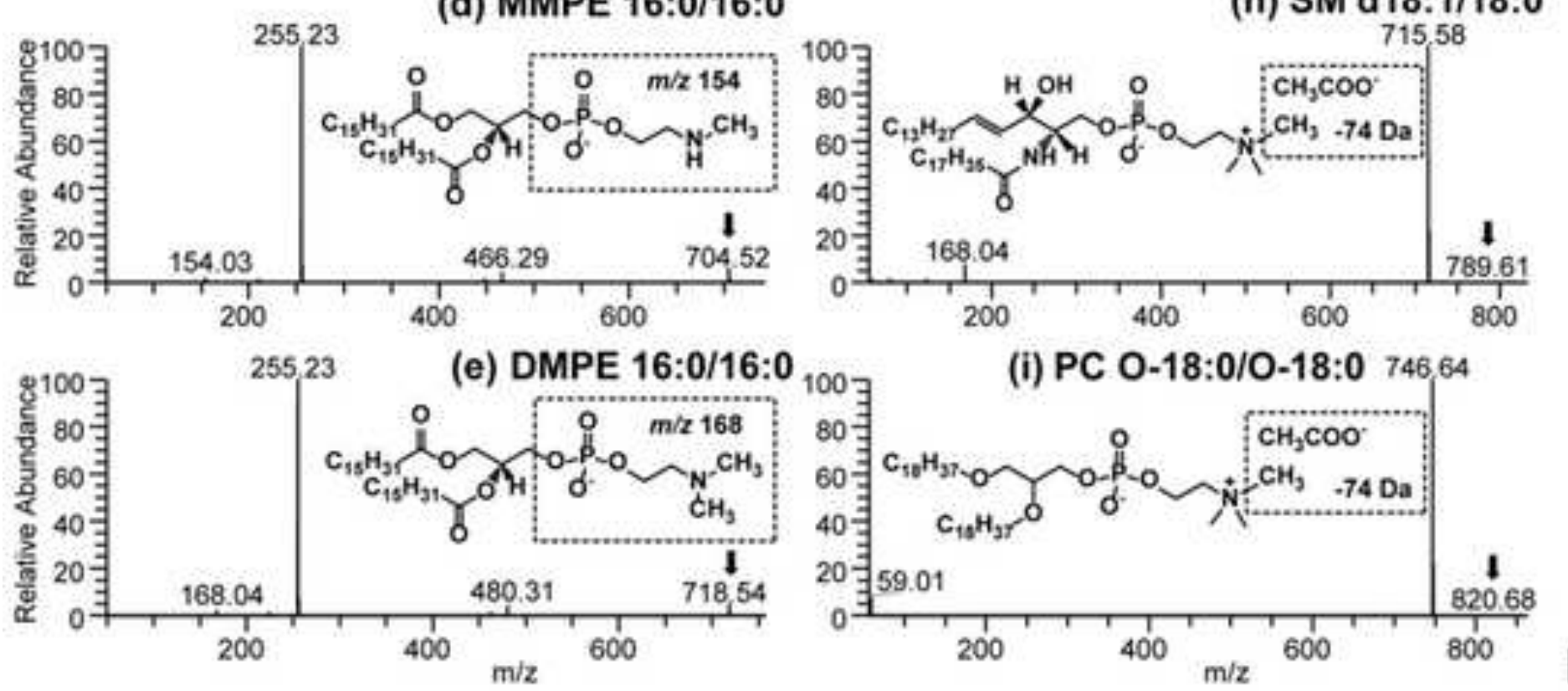
a

$$
\begin{array}{clc}
\begin{array}{c}
\text { Functional group } \\
\text { (variable) }
\end{array} & \begin{array}{l}
\text { Linker } \\
\text { (static) }
\end{array} & \begin{array}{c}
\text { Scaffold } \\
\text { (conserved) }
\end{array}
\end{array}
$$

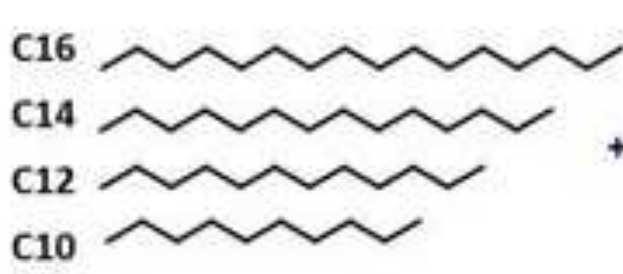

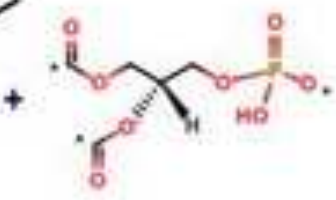

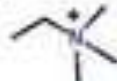

Choline

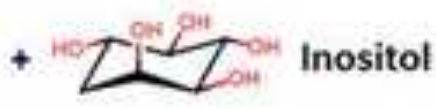

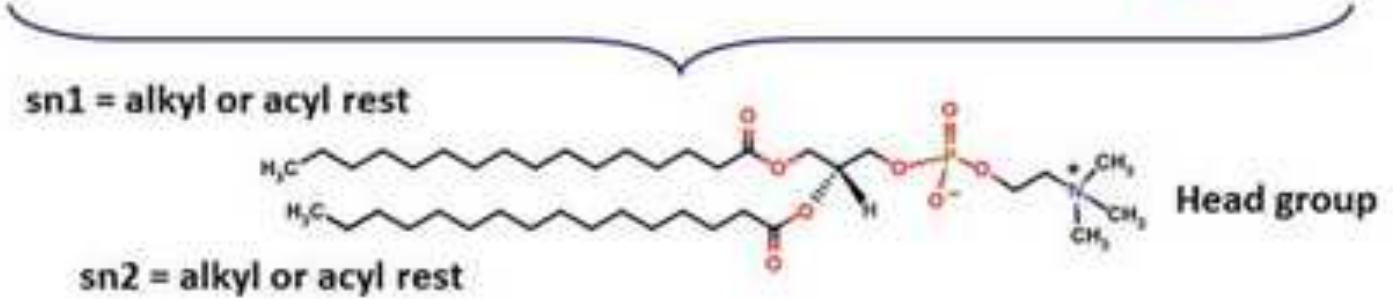

b
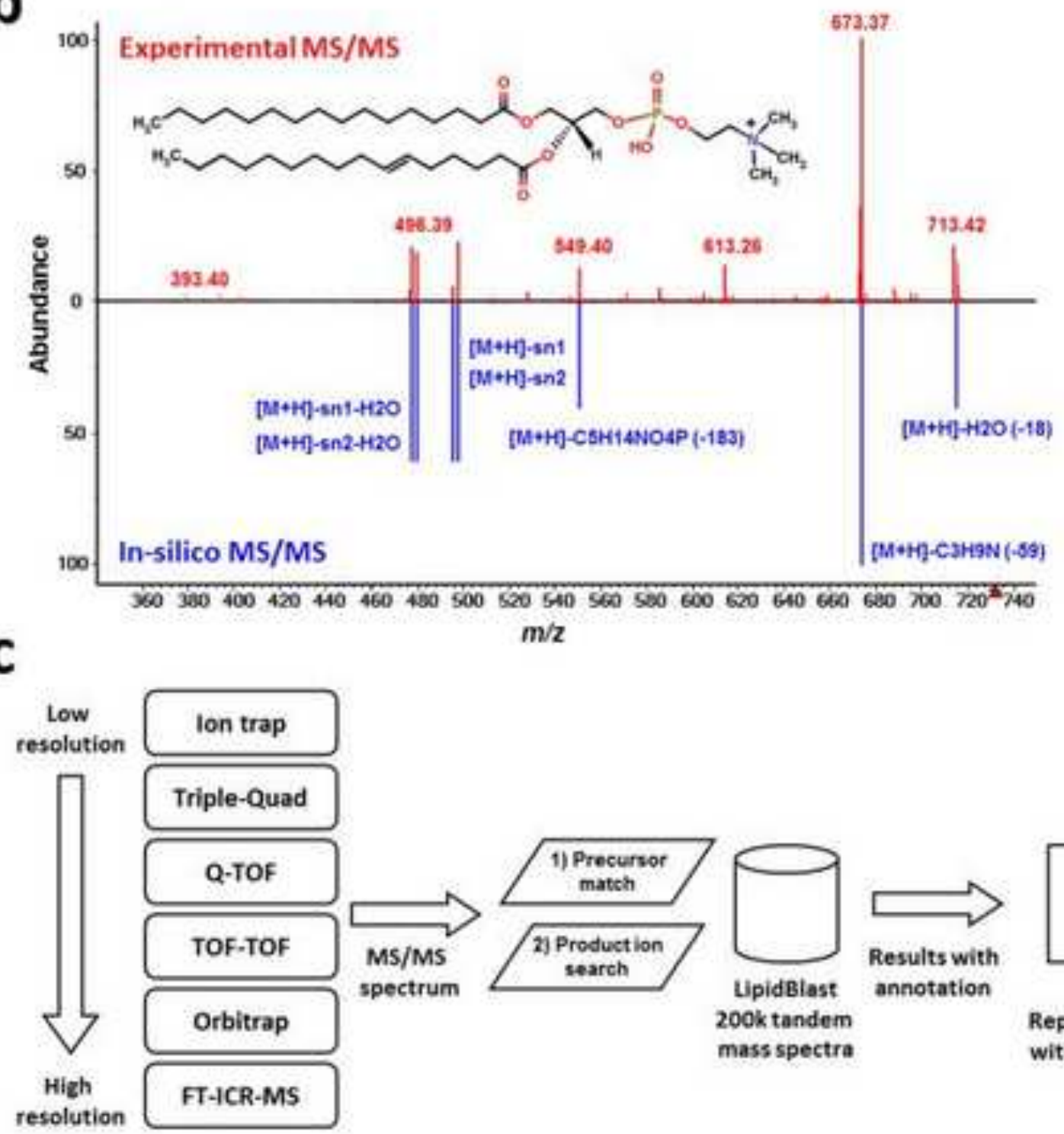

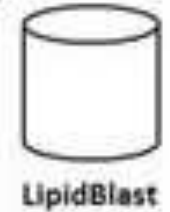

200k tandem mass spectra

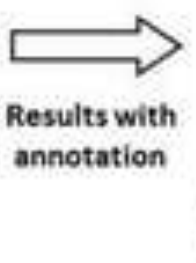

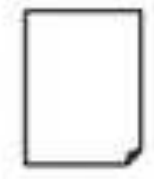

Report with with scores 
Manuscript Number:

Title: Lipidomics and recent applications in clinical and medical research with ultrahigh performance liquid chromatography-mass spectrometry

Article Type: Review Article

Keywords: Lipids, liquid chromatography, tandem mass spectrometry; clinical profiling, urine, blood.

Corresponding Author: Professor Heli Marja Marita Siren, Ph.D.

Corresponding Author's Institution: University of Helsinki

First Author: Henri F Avela, MSc

Order of Authors: Henri F Avela, MSc; Heli Marja Marita Siren, Ph.D.

Abstract: Lipids are organic biomolecules, which contribute to energy storing, cellular and subcellular membrane assembly, functionality, signalling, gene expression regulation and surfactant formation. Lipidomics comprises of identification and quantitation of organic lipids, their derivatives and variants.

The present review article combines the literature on glycero-, glycerophospho- and sphingolipids in lipidomics from the years 2017-2019. In addition, a few papers which have promoted advancement in lipidomics are discussed. The primary focus is lipid profiling of biological lipidomic systems with ultrahigh performance liquid chromatography (UHPLC) coupled with mass spectrometry (MS, tandem MS) detection, data handling, and calculations with analytical tools and current statistical methods. Research on in vivo samples includes matrix categories, such as primary blood derivatives (plasma, serum, erythrocytes, and blood platelets), faecal matter, urine, and liver tissues.

Suggested Reviewers: X L Guan Dr Professor, Lee Kong Chian School of Medicine, Nanyang Technological University, Singapore xueli.guanantu.edu.sg Specialists and professional on lipidomics.

Coral Barbas Dr Professor, CEMBIO, Centre for Metabolomics and Bioanalysis, San Pablo CEU University cbarbas@ceu.es Specialist in lipidomics and bioanalysis.

Olivier Berdeaux Dr

Centre des Sciences du Goût et de l'Alimentation, Agrosup Dijon, CNRS, INRA, Université Bourgogne Franche-Comté olivier.berdeaux@dijon.inra.fr 
Specialist in bioanalysis and metabolomics in lipidomics.

Jos F Brouwers Dr

Department of Biochemistry and Cell Biology, Faculty of Veterinary Medicine, utrecht University

J.brouwers duu.nl

Specialist in lipidomics 


\section{Cover Letter}

To

The Reviews Editor

Helsinki 11.11.2019

Dear Editor,

The paper was informed to be sent to the journal on $16^{\text {th }}$ September, 2019 and asked about considering our review article for publication. The contacted person was Dr. Gregory S Makowski, Reviews Editor, CCA.

Now the review paper is ready and its title is

\section{Lipidomics and recent applications in clinical and medical research with ultrahigh perfor- mance liquid chromatography-mass spectrometry - A review}

written by Henri F. Avela and Heli Sirén

is sent to Clinica Chimica Acta.

For this paper we have compiled the newest literature between 2017 - 2019. The articles reviewed primarily were focused on applications of clinical and medical research made with ultrahigh-performance liquid chromatographic methods with mass spectrometric (MS, tandem MS) detection. The lipid articles were considered to be sufficient enough for making the current statement of the state from glycero-, glycerophospho- and sphingolipids in lipidomics and the identification with on-line coupled liquid chromatography with mass spectrometry research. Additionally, the data handling with computational methods alongside chemometric and statistical methods are discussed. That kind of evaluation was shown to have increased importance and usage for cross-validation and data-analysis. Please, find the abstract on a separate page.

The authors declare that they do not have competing financial interest concerning the project. They do not have any conflicts, either.

The manuscript has not published in any other journal. It has ten (10) figures and eleven (11) tables.

Thank you for considering the submission. We look forward to your response.

Sincerely

Heli Sirén,

Docent (Assoc.Prof.), University of Helsinki, Finland 


\title{
Lipidomics and recent applications in clinical and medical research with ultrahigh perfor- mance liquid chromatography-mass spectrometry - A review
}

\author{
Henri F. Avela and Heli Sirén \\ Department of Chemistry, University of Helsinki, P.O. Box 55, FI-00014 University of Helsinki \\ (FI-00560 Helsinki), Finland \\ *) Correspondence: Henri F. Avela (MSc), Faculty of Science, Department of Chemistry, University \\ of Helsinki, A.I. Virtasen Aukio 1 (P.O. Box 55), FI-00014 University of Helsinki, Finland; Heli \\ Sirén (Assoc. Professor, Doc), Faculty of Science, Department of Chemistry, University of Helsin- \\ ki, A.I. Virtasen Aukio 1 (P.O. Box 55), FI-00014 University of Helsinki, Finland, \\ heli.m.siren@helsinki.fi
}

\begin{abstract}
Lipids are organic biomolecules, which contribute to energy storing, cellular and subcellular membrane assembly, functionality, signalling, gene expression regulation and surfactant formation. Lipidomics comprises of identification and quantitation of organic lipids, their derivatives and variants.

The present review article combines the literature on glycero-, glycerophospho- and sphingolipids in lipidomics from the years 2017-2019. In addition, a few papers which have promoted advancement in lipidomics are discussed. The primary focus is lipid profiling of biological lipidomic systems with ultrahigh performance liquid chromatography (UHPLC) coupled with mass spectrometry (MS, tandem MS) detection, data handling, and calculations with analytical tools and current statistical methods. Research on in vivo samples includes matrix categories, such as primary blood derivatives (plasma, serum, erythrocytes, and blood platelets), faecal matter, urine, and liver tissues.
\end{abstract}

Keywords: Lipids, liquid chromatography, tandem mass spectrometry; clinical profiling, urine, blood. 


\section{CCA AUTHOR CHECKLIST}

This form is to be submitted with the manuscript. Please fill in and check boxes below.

Article Title: Lipidomics and recent applications in clinical and medical research with ultrahigh performance liquid chromatography-mass spectrometry - A review Corresponding Author: Henri F. Avela and Heli Sirén

$\square$ Structured abstract

$\mathrm{x} \square$ Keywords

$x \square$ References are in journal format. References in text and reference list correspond exactly.

$x \square$ Quantities and units conform to international practice

If this paper is a re-submission, please include:

Article Reference Number: 

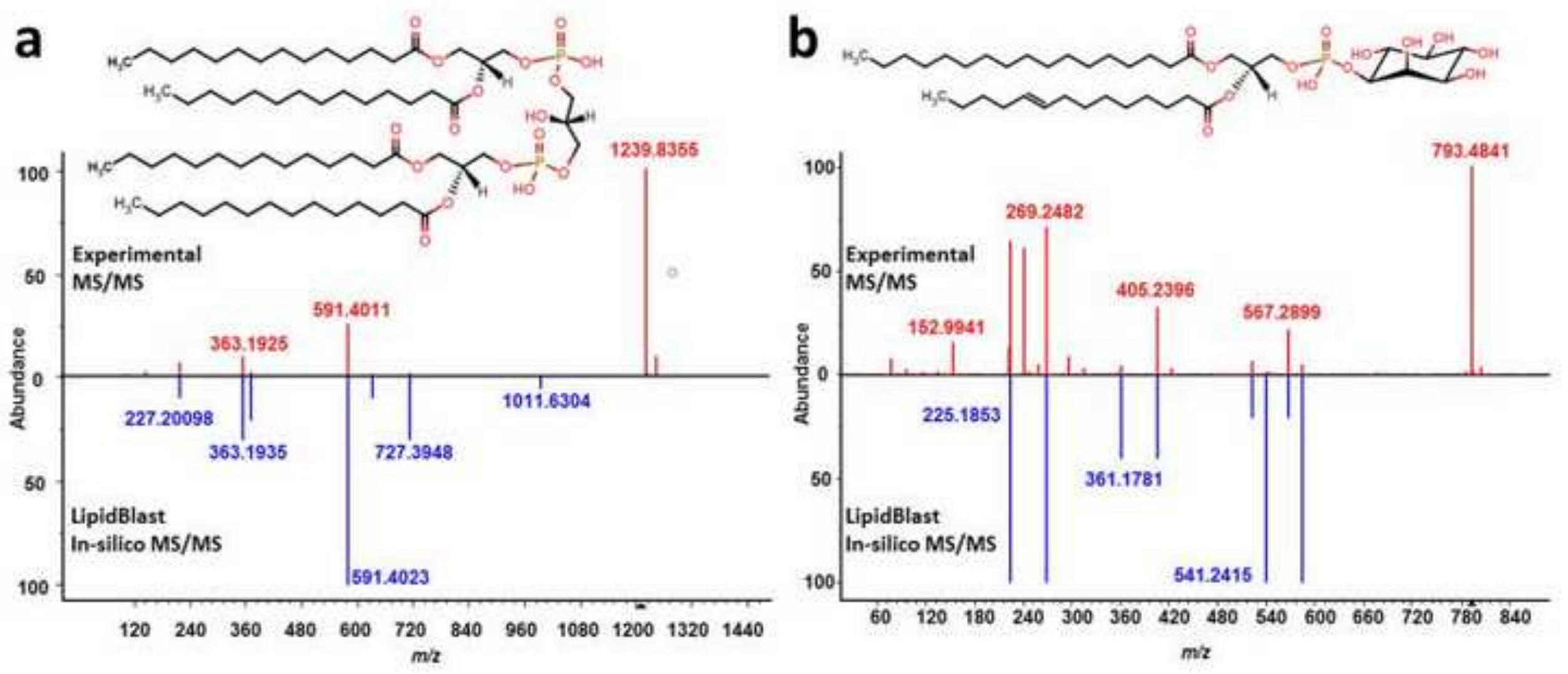